\title{
Navigating high-speed unmanned surface vehicles: system approach and validations
}

\author{
Jiayuan Zhuang, b, c , Lei Zhang ${ }^{\mathrm{a}, \mathrm{b} 1}$, Bo Wang ${ }^{\mathrm{a}, \mathrm{b}}$, Yumin Su ${ }^{\mathrm{a}, \mathrm{b}}$, Hanbing Sun ${ }^{\mathrm{b}}$, Yuanchang Liu ${ }^{\mathrm{c} 2}$ and Richard \\ Bucknall $^{\mathrm{c}}$ \\ a. Science and Technology on Underwater Vehicle Laboratory, Harbin Engineering University, Harbin, 150001, China \\ b. College of Shipbuilding Engineering, Harbin Engineering University, Harbin, 150001, China \\ c. Department of Mechanical Engineering, University College London, Torrington Place, London WC1E 7JE, UK
}

\begin{abstract}
With an increasing interest in the deployment of unmanned surface vehicles (USVs) to support complex ocean operations, high-speed USVs ( $\geq 40$ knots) have become an important option, especially in accomplishing demanding tasks such as coastal guarding. At present, there is a vast amount of studies focusing on the development of USVs' autonomous navigation systems, and the results of most of them have only been verified by simulations or demonstrations on low-speed experimental USVs ( $\leq 20$ knots). It remains unclear how high-speed USVs would perform in real applications. In particular, when USVs are navigating at high-speed, reliable obstacle detections and robust motion control become increasingly difficult to achieve than in normal conditions. This paper therefore presents a new autonomous navigation system for high-speed USVs with the system software and hardware been designed on the basis for ensuring a safe and reliable operation. Within such a system, a collision avoidance algorithm based on velocity obstacle (VO) able to adjust the USV's heading and speed in real-time has been integrated. A local environment modelling method and a heading maintain method by considering Coast Guard International Regulations for Avoiding Collision at Sea (COLREGs) are proposed to improve the reliability and stability of the system. The effectiveness and efficiency of the proposed system has been verified and validated by hardware-in-the-loop simulation tests and sea trials on a high-speed USV ('Tianxing No.1') in practical maritime environments. Results show that the autonomous navigation system adapts to the characteristics of high-speed USVs and can guide high-speed USVs ( $\geq 40$ knots) in realising a safe autonomous navigation, which significantly improves the USV's autonomy level.
\end{abstract}

Key words: unmanned surface vehicle (USV); autonomous navigation system; obstacle avoidance; high-speed USVS

\section{Introduction}

According to USV Master Plan (USA navy, 2007), unmanned surface vehicle (USV) is the vehicle that has the feature of displacing water at rest, operating with near-continuous contact with the water surface, and

${ }^{1}$ Corresponding author. E-mail: cheung103@163.com

2 Co-corresponding author. E-mail: yuanchang.liu@ucl.ac.uk 
being capable of unmanned operations with varying degrees of autonomy. Presently, USVs have become the platforms with increasing popularity in being deployed in a wide variety of maritime missions. These missions range from civil or research applications such as oceanography (Kitts et al., 2012), remote sensing, environmental monitoring (Sharma et al., 2013), surveying, to military deployments including weapons delivery, sea patrol (Bertaska et al., 2015), coastal guarding (Manley, 2008), anti-submarine warfare, surface warfare, electronic warfare and maritime interdiction operations support (Caccia et al., 2008; Xu et al., 2006; Yan et al., 2010).

A large number of USVs have been designed with representative prototypes including Protector (Israel), Spartan and Sea Hunter (America), Charlie (Italy) (Manley, 2008), Springer (UK) (Sutton, 2011) and CCAT/C-Worker (UK) (Pieterkosky et al., 2017; Iovino et al., 2018), Inspector (France), Delfim (Portugal) (Alves et al., 2006), Aragon (Korea) (Han et al., 2020). USVs can be categorised into different types according to their operating speeds as: 1) low-speed USVs ( $\leq 10$ knots), 2) medium-speed USVs (10-40 knots) and 3) high-speed USVs ( $\geq 40$ knots). Most of low-speed USVs employ catamaran platforms and an electric propulsion system with the typical platforms including Springer and Charlie. Such platforms have the advantages of low operating cost and improved control robustness. However, due to the limitations of vehicle sizes, equipped energy endurances and payload capacities, the applications of low-speed USVs are limited for inland or near-shore waters.

In recent decades, there is a trend in deploying USVs to assist with tasks of high complexity in unstructured maritime environments. These tasks require USVs to be capable of operating at a high speed to improve mission execution efficiency. For example, for a civilian application like post-disaster search and rescue, high-speed USVs are able to provide a rapid emergency response and a fast post-disaster damage assessment and inspection. Similarly, with regards to military applications, the increasing emphasis on port and littoral security has made high-speed USVs a more preferred option as they can achieve the missions of sea patrolling and attacking hostile vessels in adverse environments in a more efficient way.

It is without any doubt that the construction of a high-speed USV is different to that of low-speed USVs in both hardware and software design. In terms of hull design, high-speed USVs are normally designed as waterborne crafts with the planing being the dominant mode of operation; whereas, low-speed USVs in general 
have displacement hulls and can only be operating at displacement mode. Such a fundamental difference not only imposes a diversity in ship design of two different types of USVs but brings a challenge to the control of high-speed USVs. The planing operating mode is largely dependent upon the hydrodynamic lift that keeps vessels surfacing when USVs are travelling with high-speeds. Such a phenomenon can make the dynamic characteristics of high-speed USVs complex and the motion control of vessels is associated with high level of nonlinearity. In addition, when propelling vessels, diesel engines are normally equipped on high-speed USVs and work under the propulsion modes of water-jet (20-40 knots) or surface-drive ( $\geq 40$ knots). These two modes are different to the conventional propulsion for low-speed USVs, which either adopt rudder-propeller system or multi-propeller where the steering of the vehicle is achieved by directly changing the thrust direction. Compared with water-jet mode, surface-drive propulsion system is a more appealing option for high-speed USVs as it is able to achieve a more efficient acceleration. However, such an advantage requires a real-time adjustment of the trim of propulsion system according to the attitude of the vehicle, which makes surfacedrive more complex than the water-jet propulsion system.

To detect other static or moving obstacles, typical environmental perception sensors including marine radar, Light Detection and Ranging (Lidar), Autonomous Identification System (AIS), visible light cameras, infrared cameras and panoramic vision cameras (Oleynikova et al., 2010; Bohren et al., 2008; Urmson et al., 2008; Park et al., 2017; Han et al., 2020) are used. Note that when sailing at high-speed, USVs may experience a significant change in terms of pitch and roll angles. Also, incurred wave and wind may cause severe heave motions. All of these make it difficult for Lidar to receive effective echo in a stable way making it unsuitable for high-speed USV. In addition, the accuracy of AIS data may be affected by weather and communication conditions. Geopolitical reasons also lead to a limited adoption of AIS on ships. For example, some small fishing ships, especially in China, are not equipped with AIS devices and AIS information will not be available in military applications for security considerations. Consequently, the proposed environment perception solution in this work is not relying on Lidar and AIS but using vision systems together with marine radars.

In terms of the difference in software design between high-speed and low-speed USVs, although both platforms need to be equipped with an autonomous navigation system to support the fully autonomous operation in unstructured or unpredictable environments, there is a stricter demand in system reliability and 
real-time capability for high-speed vessels. Autonomous navigation systems reveal the intelligence level of USVs with the key functional modules being environment perception, path planning, and motion control strategies. The underlying problems with the autonomous navigation system when USVs are navigating at high speed include the instability for environment perception and vehicles' motion control. More specifically, the perceived environmental information is being updated with short interval as a USV is increasing its speed making planning response time critical to better process sensing information and subsequently make the most appropriate decisions. In addition, due to the dynamic characteristics of high-speed USVs, a deterministic control strategy should be adopted to avoid any large-scale heading change that is beyond vehicle's capacity.

To provide a solution to abovementioned problems for navigating high-speed USVs, in this paper, a realtime and reliable autonomous navigation system has been proposed. The system has a generic system architecture that can be employed upon any types of high-speed USVs subject to minor parameter adjustments (such as vehicle's dynamic characteristics or payload) according to specific platforms. Within the system, a new obstacle avoidance approach tailored for high-speed USVs has been developed such that a good collision avoidance performance can be achieved in complicated maritime environments with clustered obstacles. To verify and validate the autonomous navigation system, a planing high-speed USV named as 'Tianxing No.1' has been designed and built with the primary aim being hydrology and meteorological information collection, ocean mapping, environmental observation. 'Tianxing No.1' has the maximum speed of 50 knots and cruising speed of 40 knots. Powered by a diesel-electric hybrid system, 'Tianxing No.1' has a long endurance capability with the maximum range being $1000 \mathrm{~km}$. A series of sea trials carried out in various open sea areas in China has well demonstrated the effectiveness of 'Tianxing No.1' as well as its designed autonomous navigation system.

Highlights of this paper are:

(1) When a USV is navigating at high-speed, the unsteady motion can potentially make the detection of obstacles instable. Also, the motion control under such a situation is more difficult than in normal conditions. The autonomous navigation system proposed in this paper primarily addresses these issues and provide a real- 
time and reliable navigation solution from both software and hardware perspectives.

(2) To resolve the problem of obstacles' instability, especially in the absence of AIS information, a visionradar based local environment model generation method has been designed to ensure the stable detection of static obstacles.

(3) To satisfy the safety requirements of autonomous navigation system, a heading maintaining method with the consideration of COLREGs has been integrated into the collision avoidance module.

(4) The effectiveness and efficiency of the proposed system has been verified and validated on a newly built practical high-speed USV, named 'Tianxing No.1'. Extensive sea trials have been conducted in practical maritime environments, i.e. Shenzhen Bay Port at Guangdong province, China and New Lvshun Port at Dalian, Liaoning province, China. The practicability of this work can be further reflected in that all obstacles in this work are actual ships of different types and sizes. This is different to most existing literature where moving obstacles are manually controlled target ships.

The rest of the paper is organized as follows. Section 2 reviews the state-of-the-art of USVs as well as key technologies in autonomous navigation and motion planning algorithms. Section 3 presents the software and hardware architectures of the proposed high-speed autonomous navigation system. Section 4 specifically introduces the new obstacle avoidance algorithm based on velocity obstacle (VO) to address the problems for high-speed USV autonomous navigation and increase the reliability and stability of autonomous navigation systems. Details of 'Tianxing No.1' together with the experimental validation of the proposed autonomous navigation system are discussed in Section 5. Section 6 concludes the paper and discusses the future work.

\section{Literature review}

The design of an appropriate autonomous navigation system, especially for high-speed USV, requires significantly amount of computational resources, which might not be an ideal option for the real-time navigation where fast response times are preferred (Lee et al., 2015). A solely vision-based solution for a high- 
speed USV was presented in Wang et al. (2011). They design a vision-based obstacle detection system for USV towards the aim of real-time and high-performance obstacle detection on the sea surface. Sea tests have been taken and the obstacle detection system for USV is proven to provide stable and satisfactory performance. Hermann et al. (2015) proposes a sensor fusion strategy of radar and vision technologies further supplemented with dedicated filtering and statistical methods to obtain robust results for marine operations of highly maneuverable and fast planning crafts. Almeida et al. and Martins et al. $(2009 ; 2007)$ use marine radar and other sensors to detect obstacles and for collision avoidance on Portuguese ROAZ II. Oleynikova et al. (2010) use Medea as a verification platform to detect targets from radar images, and on the basis of this, they discuss plan waypoints, routes, complete perimeter patrol, and laser radar in USV applications (Bohren et al., 2008; Urmson et al., 2008; Haltenman et al., 2010; Holler, 2008). Zhuang et al. (2016) develop an embedded collision avoidance system based on marine radar and the system is verified by sea trial results. Korea Advanced Institute of Science and Technology (KAIST) develop a USV for 2014 Maritime RobotX Challenge, the USV hardware was developed on a general catamaran platform by propulsion, sensors, computer, power, and emergency systems, by combing with the software five mission tasks can be completed, i.e. navigation and control, underwater search and report (Park J. et al. 2017).

To support the path planning functionality of USVs, a hierarchical strategy-based path planning approach is normally adopted with such an approach consisting of global path planning and local obstacle avoidance algorithms (Casalino et al., 2009). The local obstacle avoidance algorithms are mainly divided into two categories, i.e. the path searching based local obstacle avoidance algorithms (Larson et al., 2006), and the behavior based local obstacle avoidance algorithms (Huntsberger et al., 2011).

The path searching based algorithms are usually applied to the low-speed USV, for that the flexibility is poor and cannot cope with the obstacle avoidance problem quickly. Among different types of algorithms, a rapidly exploring random tree (RRT) algorithm that has been designed to efficiently search for a path in nonconvex and high-dimensional spaces, is normally used. Because of the feature of not required to explicitly set any resolution parameters, RRTs are capable of exploring the environment space rapidly and uniformly using a random sampling scheme. However, RRTs are not suitable for dynamic path planning which requires 
to avoid moving obstacles as the algorithm is incapable of providing a global optimal solution with the least distance cost (Lolla et al. 2014). Alternatively, the fast marching method (FMM) with its improved versions have been used as they are able to generate a smooth trajectory within sufficient time (Liu and Bucknall, 2015; Song et al., 2017). In addition, the Evolutionary Algorithm (EA) has been largely adopted to search for the feasible navigation route which is compliant with the COLREGs (Tam and Bucknall, 2010; Tsou and Hsueh, 2010). It should be noted that a common problem for EA based approaches is the discontinuity of the paths which makes paths difficult for vehicles to follow and consequently may not be able to guarantee the safety navigation. Naeem et al. (2012) designed a COLREGs compliant path planner by using a modified A* algorithm. Kim et al. (2015) proposed a novel angular rate constrained algorithm based on the Theta*, which is a grid-based method similar to $A^{*}$. Singh et al. (2020) integrated a constrained $A^{*}$ approach with a decentralised virtual target (VT) guidance approach combined with a potential field based swarm aggregation technique for the cooperative navigation of multi USVs. Mina et al. (2020) proposed method integrating offline optimal path planning with a safety distance constrained A* algorithm, together with an online extended adaptively weighted (EAW) artificial potential field-based path following approach with dynamic collision avoidance, based on USV manoeuvring response times. It should be noted that the processes of gridbased map construction and path searching require a great deal of time with the grid-based world model needed to be updated according to the change of sensor data. Meanwhile, these path planning methods lack flexibility, which in particular makes USVs' collision avoidance capability compromised when vehicles are travelling at a high-speed in environments with dense moving obstacles (Thakur et al.,2012).

When it comes to the path planning problem, it is important that a number of factors should be taken into account, including the depth, the traffic density, the obstacle-avoidance, the mainstream direction of the traffic flow (Ma et al. 2018). Niu et al. (2020) proposed a Voronoi-Visibility-GA energy efficient (VVGAEE) energy efficient path planning algorithm, but it is not suitable for real-time collision avoidance. Different to conventional path searching algorithms, with the recent advance in Artificial intelligence (AI), the machine learning (reinforcement learning in particular) based algorithms have been widely used for autonomous navigation for unmanned ground vehicles (UGVs) (Sombolestan et al., 2019; Saha and Dasgupta, 2017), 
unmanned aerial vehicles (UAVs) (Choi \& Cha, 2019). In terms of ocean domain, Yoo and Kim (2016) considered the effect of ocean currents and propose a path planning algorithm for unmanned marine vehicles. Chen et al. (2019) proposed a novel approach of ship path planning based on the Q-learning algorithm and Nomoto model, the approach was validated and compared with the A* and RRT algorithms, but no other dynamic obstacles were considered in the simulation. Guo et al. (2020) combined deep deterministic policy gradient (DDPG) and artificial potential field (APF) to proposed an improved Deep reinforcement learning (DRL) method for USV path planning, the data were acquired based on electronic chart and trained in combination with the COLREGs, this method was only realized by simulation and not considered the ship's motion model and actual verification environments. Most of these methods based on the known or structured environmental information, requiring a long training period and a large amount of computation resources making reinforcement learning based methods difficult to meet the real-time and reliability requirements of high-speed USVs.

The behavior-based local obstacle avoidance algorithms have the characteristic of fast response making them widely used for local obstacle avoidance of USV. Larson et al. (2007) used the Morphin algorithm which was proposed by Simons et al. (1998). to deal with the local static obstacle avoidance of USVs. Morphin is an area-based approach which analyzes patches of terrain to determine the traversability of each patch, and evaluates the traversability of a path by determining the set of patches it travels through. Tang et al. (2015) developed a behavior-based reactive local obstacle avoidance algorithm using the concept of dynamic window such that the behavior of dynamic obstacle within a certain time can be considered leading to a better avoidance strategy. A low-resource path-planning and obstacle avoidance algorithm called GODZILA (GameTheoretic Optimal Deformable Zone with Inertia and Local Approach) (Krishnamurthy and Khorrami, 2008) was proposed for USVs, and the performance of this method was verified only by a simulation platform in a laboratory. GODZILA includes two components, a local straight-line planner utilized if the target is visible and navigation towards a random target. Since the algorithm follows a local approach, it is possible to be caught in a local minimum. When a local minimum or a 'trap' is detected, navigation towards a random target is initiated to escape the trap. Fiorini and Shiller (1998) proposed the velocity obstacle (VO) concept to realise 
real-time motion planning of a robot in dynamic environments. They applied this method for online collision evaluation and moving obstacle avoidance, as well as real-time nonlinear velocity obstacle planning. VO approaches generate a cone-shaped obstacle in the velocity space (hence the name velocity obstacles) and ensure that there will be no future collisions as long as the robot's velocity vector is outside the VO. VO makes a first-order (i.e., linear) prediction, and the collision check is done in the velocity space. Since a single collision check accounts for collision checks at all future times (due to the linear velocity assumption), VO is very fast to compute and extends well to high-speed operations with short reaction time. Furthermore, its simplicity is suited for our behavior-based control architecture (Kuwata et al. 2014). Other studies also investigated and applied the VO avoidance theory extensively (Shiller, 1999; Gal et al., 2009; Zhuang et al., 2019). Song et al. (2018) combined the VO with the APF method to implement the collision avoidance that according to the COLREGs and verified by simulation tests. Bandyophadyay et al. (2010) proposed a type of reactive obstacle avoidance algorithm based on VO for USVs. This algorithm was verified by USV kayaks with a maximum velocity of 5 knots in the Singapore harbour. Bertaska et al. (2015) designed a planner which combines a local search based on the VO concept with a global, lattice-based search for a dynamically feasible trajectory, the planer was demonstrated by on-water tests by different USVs under 5 knots.

Based on the performance and applications of each obstacle avoidance algorithms, and by considering the high-speed USV obstacle avoidance requirements, dominant obstacle avoidance algorithms are compared against to some key factors such as real-time, reliability, environmental information and etc. As shown in Table 1, most of path searching based algorithms, such as RRT, FMM, EA are implemented in known environment, and could not meet the real-time and reliability requirements for high-speed USVs. High-speed USVs are normally deployed in unknown open waters with its working environment relatively simple, thereby making the behavior-based obstacle avoidance algorithms more promising. Considering the factors of algorithm's reliability and scalability, VO has its evident advantages and can be applicable for solving avoidance with moving obstacles. Therefore, VO has been selected as the basic algorithm for the obstacle avoidance in this work. 
TABLE 1 Comparison of different obstacle avoidance algorithms.

\begin{tabular}{cccccc}
\hline & $\begin{array}{c}\text { Real- } \\
\text { time }\end{array}$ & Reliability & $\begin{array}{c}\text { Environmental } \\
\text { information }\end{array}$ & $\begin{array}{c}\text { Environmental } \\
\text { complexity }\end{array}$ & $\begin{array}{c}\text { Applicable } \\
\text { to moving } \\
\text { obstacles }\end{array}$ \\
\hline RRT & Medium & Medium & Known & Complex & Unsuitable \\
FMM & Low & Medium & Known & Complex & Suitable \\
EA & Low & Low & Known & Complex & Suitable \\
A* $^{*}$ & Low & High & Known & Complex & Unsuitable \\
AI & Low & Low & Known & Complex & Unsuitable \\
Morphin & High & Medium & Unknown & Simple & Unsuitable \\
GODZILA & High & Low & Unknown & Medium & Unsuitable \\
VO & High & High & Unknown & Simple & Suitable \\
\hline
\end{tabular}

\section{System overview of autonomous navigation systems for high-speed USVs}

Central to the development of an autonomous navigation system, especially for high-speed USVs, it is important to ensure vehicle's safety, reliability and stability during the navigation. A general working procedure of an autonomous navigation system includes obtaining vehicle's own motion states as well as external environment information through the processing of sensor information, making reasonable decisions based upon perceived information and generating proper control command for the propulsion system. The performance of navigation system has a direct effect on the success of mission and by referring to the working procedures, system architectures of both software and hardware have been proposed as shown in Fig. 1 and 2. It should be noted that to make such a navigation system compatible with different platforms, system's compatibility, versatility and scalability have been addressed by constructing the system in a modular way so that algorithms within each module can be tailored according to specific platforms and applications.

\subsection{Software architecture}

The software architecture is designed in a hierarchical way with four different layers coordinating and interacting with each other. Four layers include: 1) the perception layer, 2) the control layer, 3) the communication layer and 4) the execution layer (shown in Figure 1a). A top-down control strategy from perception layer to execution layer is used guide the normal operation of USVs. In addition, to fully address the problem of limited response time of high-speed USVs, especially within emergency situation, i.e. a 
suddenly emerged obstacle is within the close range of a USV, an additional behaviour response strategy will be triggered such that the perception layer will directly send instructions to the execution layer. The software architecture is based on VxWorks (a Real-Time Operating System) and the programming language is based on $\mathrm{C}$ language. Details of each layer will be discussed in the following sections.

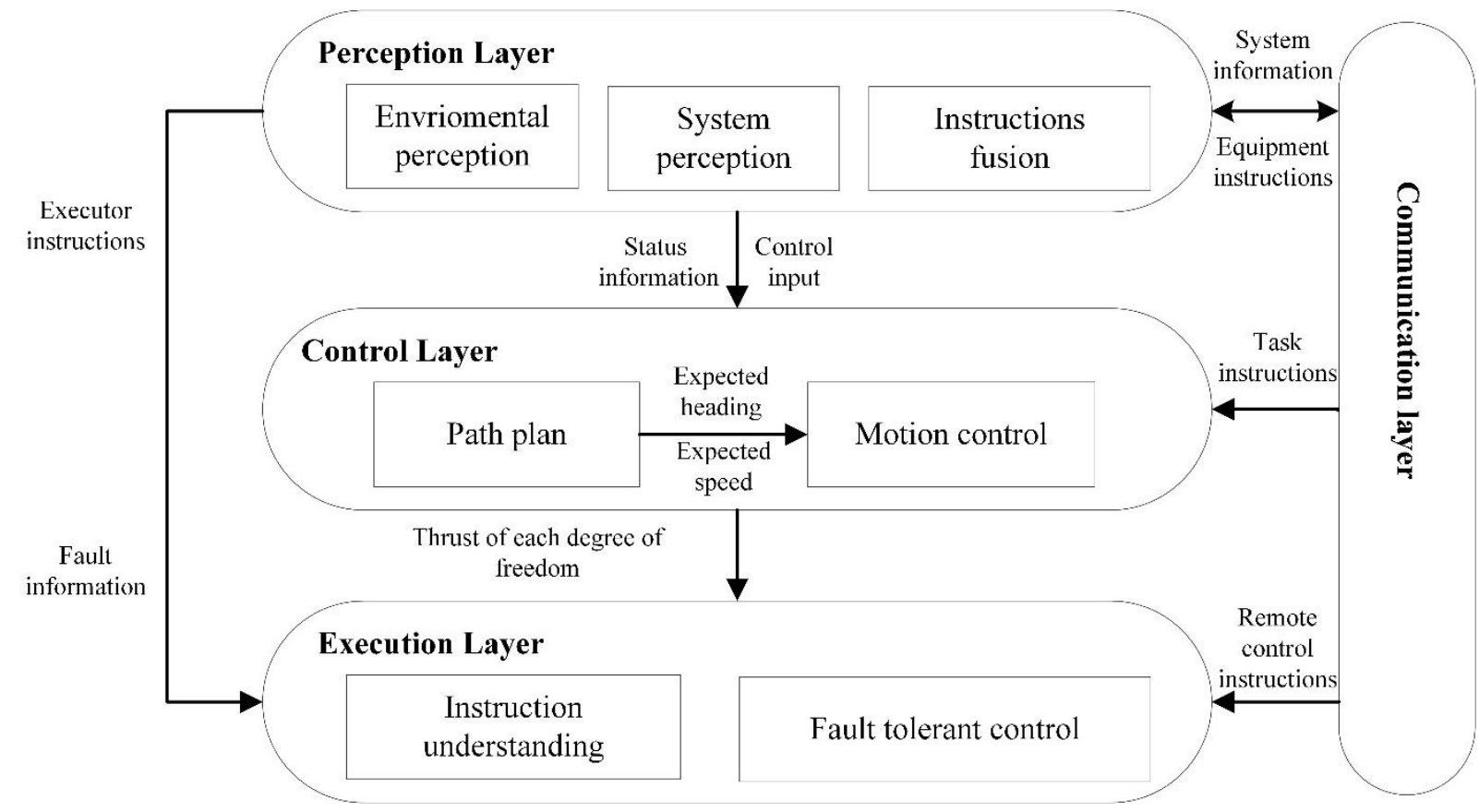

(a)

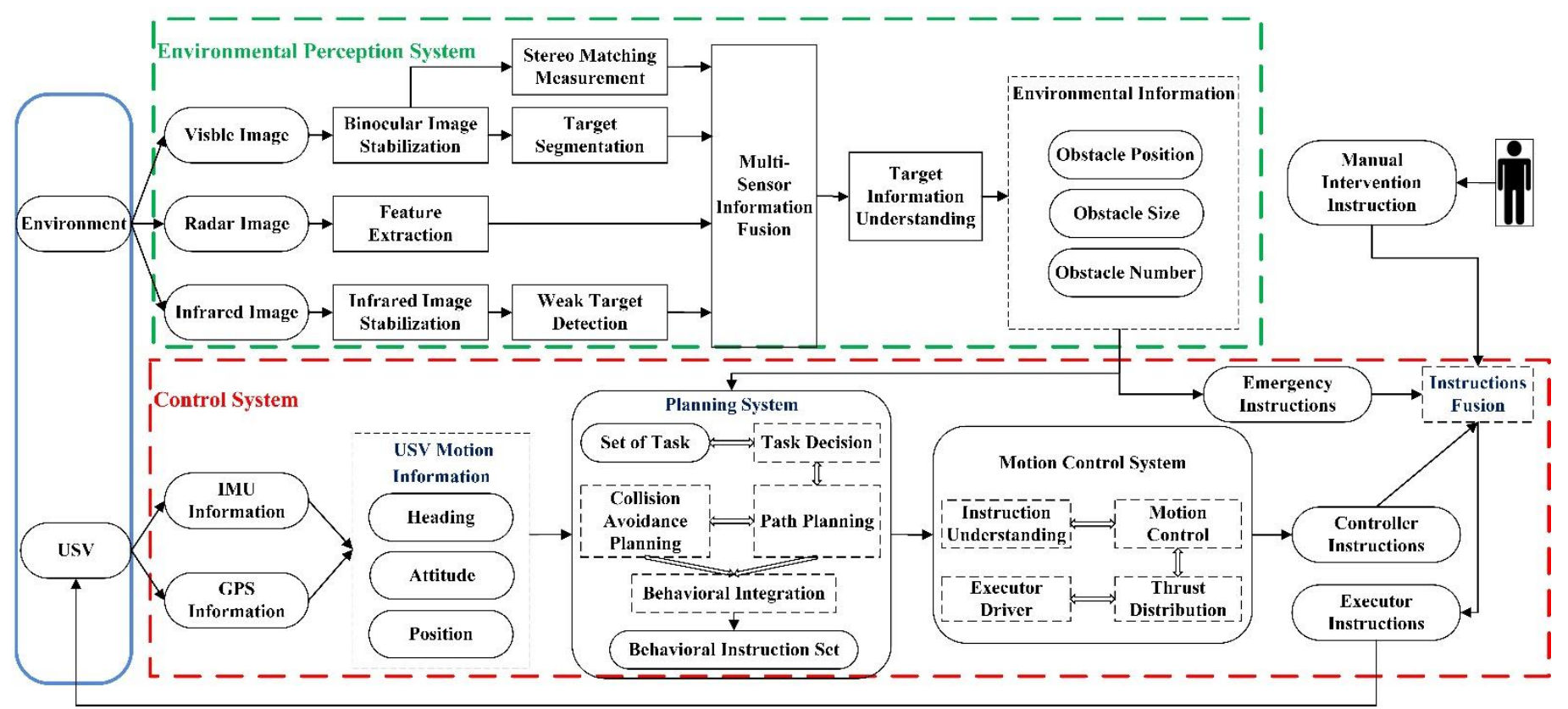

(b) 
FIGURE 1 Autonomous navigation system software structure and its working flowchart. (a) The proposed software architecture of autonomous navigation system. (b) Working flowchart of autonomous navigation system

\section{(1) Perception layer}

As shown in the perception layer illustrated in Figure 1a, the layer has the access to various onboard sensors and by using information processing algorithms, a synthetic understanding of the environment, the system (such as vehicle's states) and the status of each sensor can be obtained. Equipped sensors include GPS and INS to provide localization information, weather station to acquire the motion information and radar, electro-optical pod, panoramic vision camera to perceive the surrounding environment information, and these sensors will be discussed in detail in next section.

Figure $1 \mathrm{~b}$ provides a working flowchart describing how the navigation system works. At this stage, the perception layer is regarded as the environmental perception system as shown in the dashed green block; whereas, the control layer and the execution layer belong to the control system as shown in the dashed red block. The communication layer is responsible for information exchange among different blocks in two systems. The environmental perception system acquires radar, visible and infrared image from surrounding environment. By using algorithms for image processing, multi-sensor data fusion and target information understanding, obstacles positions, sizes and quantity can be acquired and provided to the planning system within the control system.

\section{(2) Control layer}

The control layer consists of two parts, i.e. path planning and motion control (shown in Figure 1a). The path planning system integrates USV's and its surrounding environment information from the perception layer and the mission information from communication layer, then generates behavioral instruction sets (the expected heading and speed) using path planning and obstacle avoidance algorithms (Figure 1b).

The motion control system converts the behavioral instruction sets into the thrust in each degree of freedom (DoF) domain by thrust distribution and control algorithm, then sends the thrust commands to the 
execution layer to propel vehicles (Figure 1b).

\section{(3) Communication layer}

The communication layer is acting as the intermediary responsible for the 'human-machine' interaction and the monitoring interface of various information states of the USV. Manual intervention instructions received by the USV and the status information of the feedback are encoded and processed within this layer. The data transmission and instructions interaction can be completed simultaneously to make sure the system is of high real-time capability.

\section{(4) Executive layer}

The function of the executive layer is to distribute the thrust generated from the control layer to each actuator, and subsequently propel the actuator to generate the required forces for movement. In addition, to better deal with emergency situations where a rapid respond is required, the execution layer can directly execute the instructions transmitted from the perception layer without the interference of the control layer. At the same time, fault tolerant control has been considered in this system with the thrust redistribution scheme adopted where an actuator redundant configuration can be triggered when an actuator fails (Figure 1a).

At this juncture, to summarise the designed software architecture of the autonomous navigation system, four different functionality levels form a hierarchical architecture that can communicate directly in various occasions. Especially, when the perception layer perceives some emergency situations, a fast and direct communication between the perception layer and the execution layer can be effectively formed, which is capable of solving the shortcoming of the slow response of a hierarchical system. In order to meet the realtime requirements of high-speed USV autonomous navigation system, proper information updating frequency should be used, and it is recommended here that when the USV is navigating at the speed more than 40 knots the frequency should be configured at $2 \mathrm{~Hz}$.

\subsection{Hardware architecture}


Based upon the working flow of the software architecture, a hardware architecture that reveals how the onboard equipment is arranged and interacted has been designed as shown in Figure 2. Figure 2a is the holistic architecture that has been designed for 'Tianxing No.1' high-speed USV platform with Figure 2b revealing the specific installation locations of equipment on the platform. The hardware architecture can be divided into five sub-systems as the control system, the environmental perception system, the communication system, the monitoring system and the mission load system. Within the hardware architecture, the most critical parts are the control system and environmental perception system (information being communicated within these systems is illustrated in Figure 1b). These two systems are working in collaboration as human's visual system as well as the central brain system to seamlessly process the acquired environment information and make the appropriate decisions on supporting the mission of autonomous navigation.

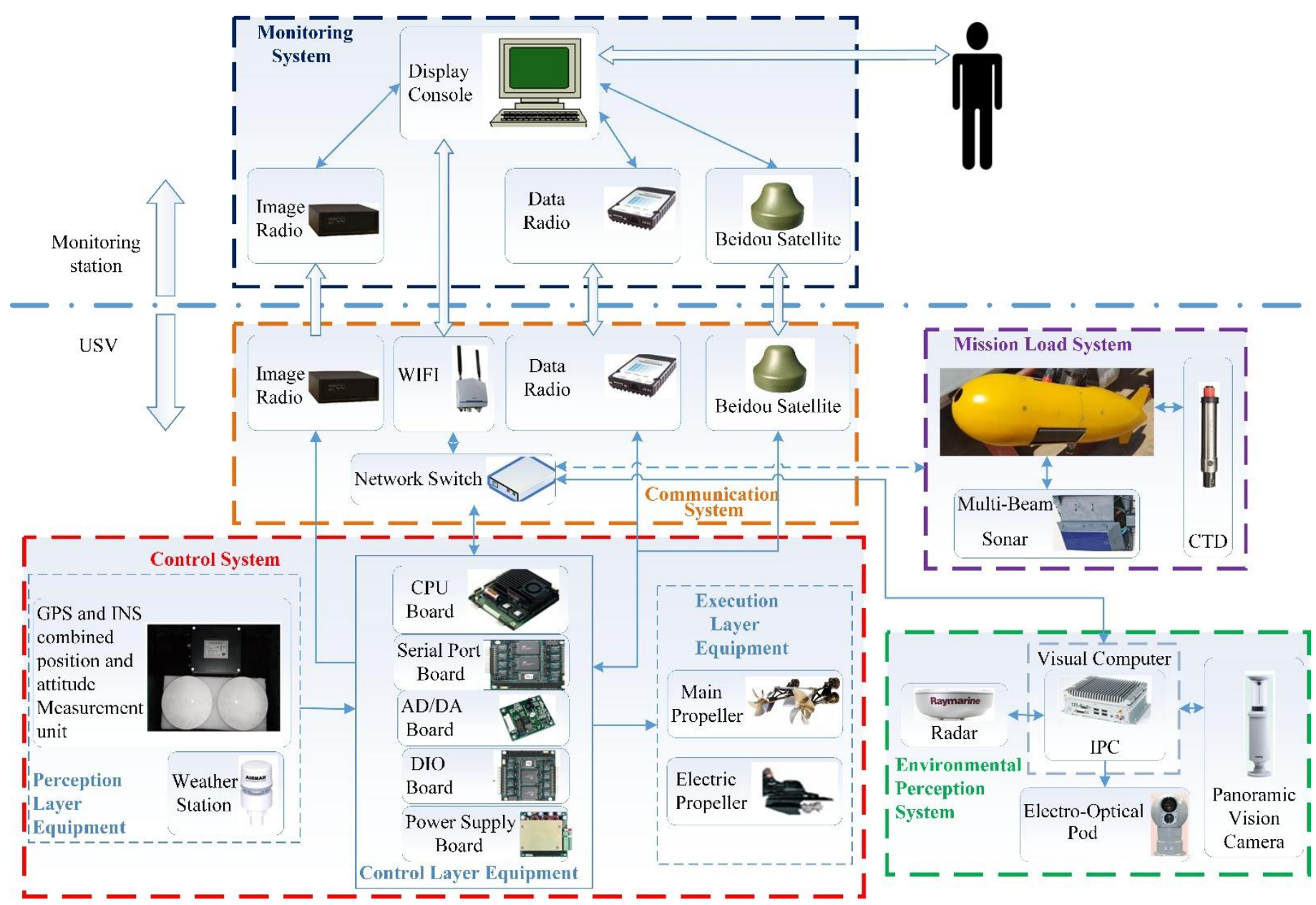

(a) 


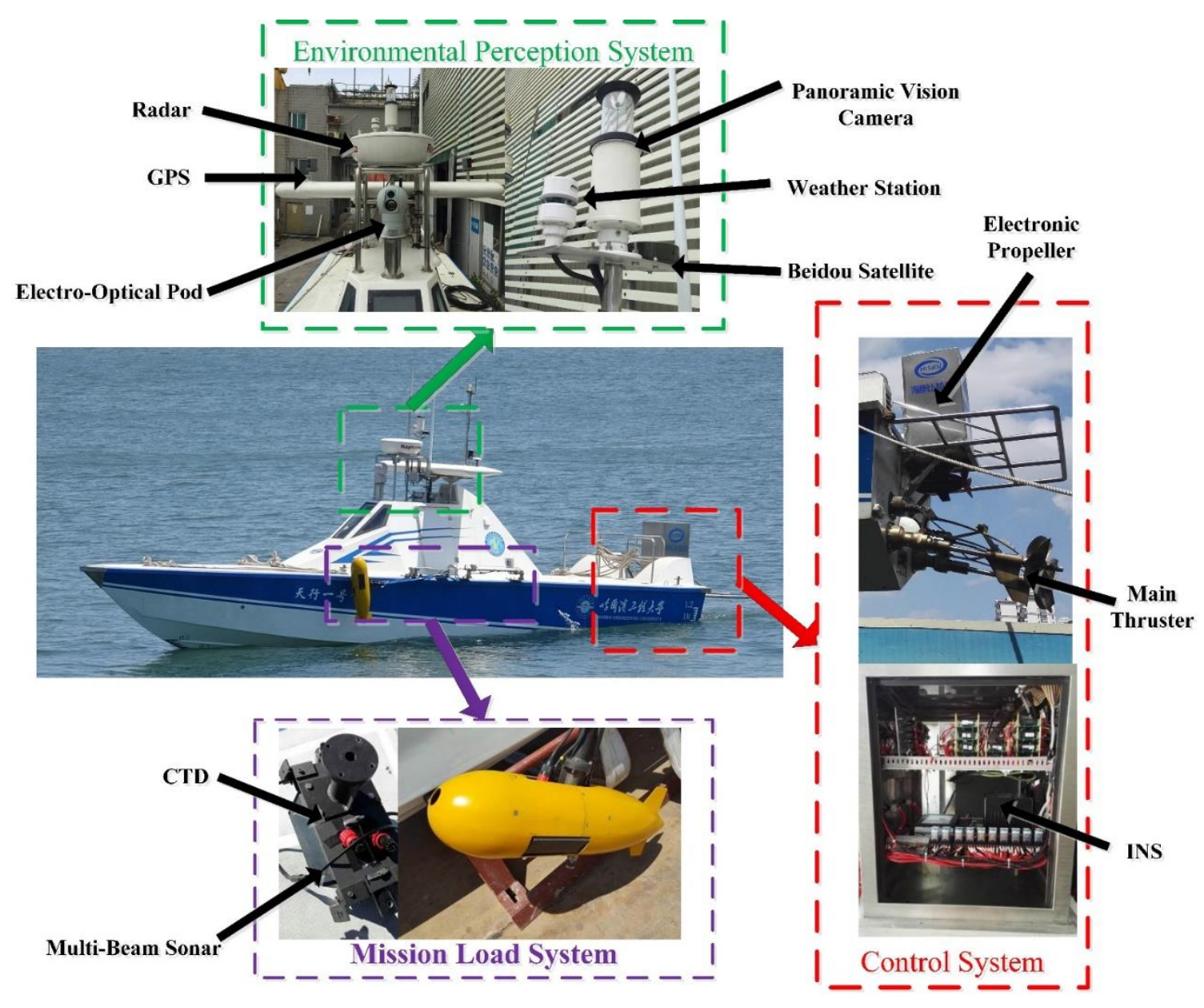

(b)

FIGURE 2 Autonomous navigation system hardware structure. (a) Autonomous navigation system general structure. b) Autonomous navigation system epuipments composition

\section{(1) Control system}

On 'Tianxing No.1' USV, main hardware equipment with the control system include: 1) a multi-board embedded industrial computer using PC104 bus as the core control computer, 2) GPS and INS combined position and attitude measurement unit, 3) weather station and 4) automatic control device of propellers. The control computer consists of the CPU board of integrated Celeron (M) processor, an 8-channel serial port board, an AD/ DA board, a DIO board and a power supply board providing 5V voltage based on PC104 bus (Figure 2a). The GPS and weather station are mounted on top of the USV mast to avoid any signal occlusion and the control computer together with INS are installed in the USV hull (Figure 2b).

The control computer processes the environmental information that is related to the motion of USVs, 
such as wind speeds and directions and the vehicle's motion information such as the position and the attitude of USV. Meanwhile, the control computer also has the access to the information in regard to the obstacles, which can be detected by visual computers.

\section{(2) Environmental perception system}

The environmental perception system consists of a visual computer, an electro-optical pod, a radar and a panoramic vision camera. The electro-optical pod contains visible light camera and infrared camera. Radar, visible light camera and infrared camera collect image information in the marine environment in real time, and fuse different types of image data to obtain an obstacle information and send this information to the control system through the communication system (Figure 2a).

On the 'Tianxing No.1' USV, the electro-optical pod and radar are mounted on top of the USV superstructure to avoid any occlusion with other equipment. The vision computer is also installed in the USV hull. (Figure 2b).

\section{(3) Communication system and monitoring system}

The communication system and the monitoring system are installed on a USV and a shore-based monitoring station, respectively. The communication system mainly supports three communication modes including network, radio and satellite communication. Data exchange between USVs and monitoring stations can be completed by the means of data radio, image radio, Beidou satellite and WIFI. In addition, the communication system also contains a communication hub that provides a $100 \mathrm{Mbp}$ communication bandwidth for all equipment (Figure 2a).

\section{(4) Mission load system}

The mission load system is designed and configured according to the specific assigned missions to the USV. For example, one of the main application areas of 'Tianxing No.1' is for environmental monitoring such as the seabed topographic mapping and the marine hydrological information survey, the onboard mission load 
system therefore is equipped with a multi-beam sonar and a conductivity, temperature and depth sensor (CTD) (shown in Figure 2a). The multi-beam sonar and the CTD are integrated into a streamlined dome, which can be retracted remotely by an auto-retracting unit (Figure $2 b$ ).

\section{Reliable real-time collision avoidance for high-speed USVs}

Compared with other types of unmanned vehicles such as UAVs, UGVs or even mobile robots, the manoeuvrability of USVs is largely limited by the vehicles' dynamic characteristics. Such a problem is especially important for high-speed USVs and requires a sophisticated collision avoidance strategy for USVs to avoid obstacles in real-time en route. By carrying out a series of sea trials, it has been found that the preferred response time for 'Tianxing No.1' high-speed USV should be less than $0.5 \mathrm{~s}$ when the vehicle is travelling with a speed over 40 knots. Such a requirement imposes a strict demand on the developed collision avoidance algorithm making the conventional grid-based algorithms largely compromised. In this paper, the velocity obstacle strategy has been adopted and modified for our USV platform and the details of the developed algorithm will be discussed in the following sections.

\subsection{Collision avoidance using velocity obstacle (VO)}

In general, velocity obstacle (VO) concept calculates the relative velocity between a vehicle and obstacles and generate a cone-shaped obstacle in the velocity space to ensure that there will be no future collisions as long as the vehicle's velocity vector is outside the VO. When VO is being applied on USVs application, such a strategy can be reflected as follows. 


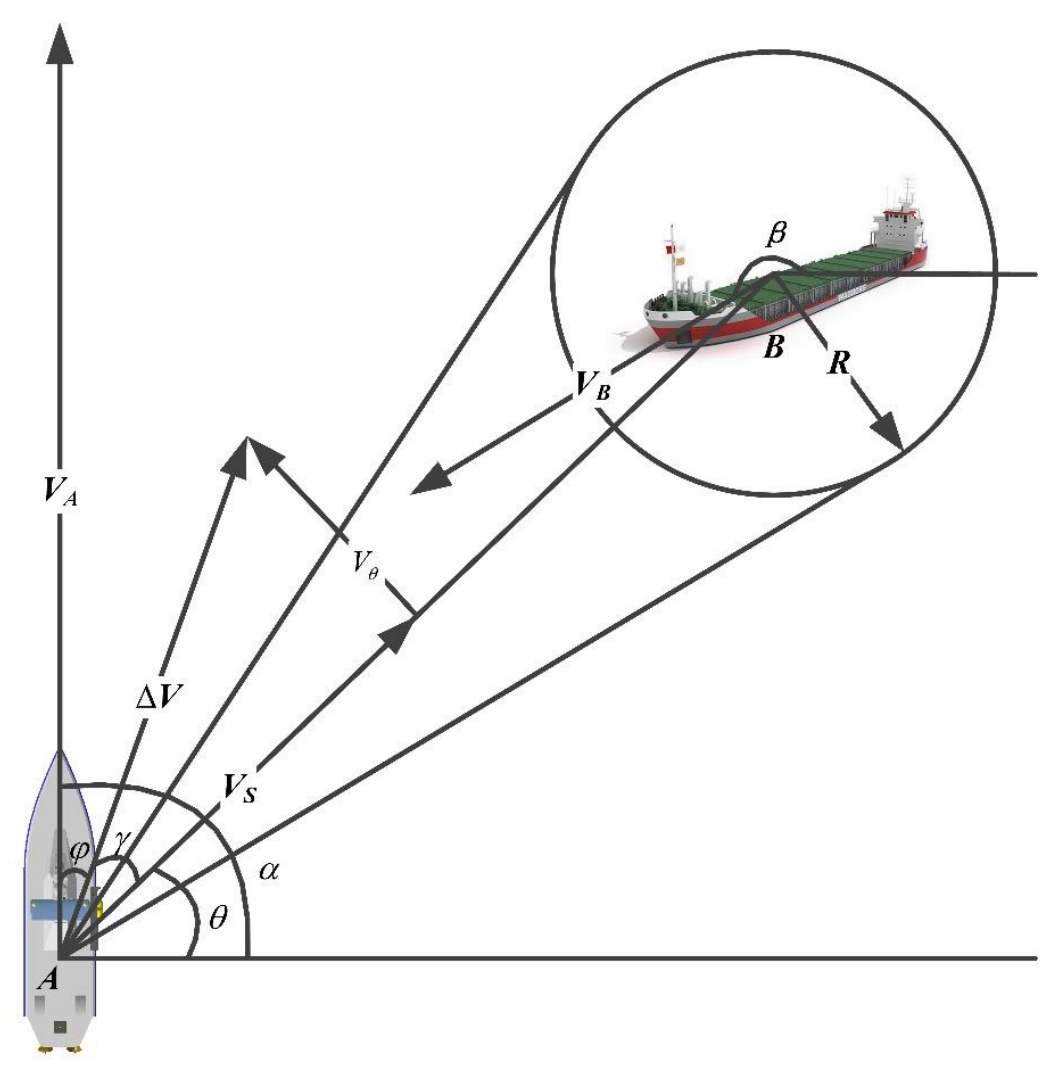

FIGURE 3 The collision model of USV and obstacle

In Figure 3, point $A$ represents a USV and an obstacle is depicted as a region of a circular shape $B$ with a radius $R$ representing its safety range that should not be violated at any time. The velocities of $A$ and $B$ are denoted by $V_{A}$ and $V_{B}$, and the heading of $A$ and $B$ are denoted by $\alpha$ and $\beta$, respectively. To calculate the relative velocity ( $\Delta V$ ) of $A$ with respect to $B$, the Light of Sight (LOS) principle can be first adopted with the decomposed velocities of $V_{\mathrm{s}}$ and $V_{\theta}$, where $V_{s}$ and $V_{\theta}$ are the relative components along and perpendicular to the LOS, respectively. Angle $\gamma$ is the angle between $\Delta V$ and LOS, and $\varphi$ is the angle between $\Delta V$ and $V_{A}$. 


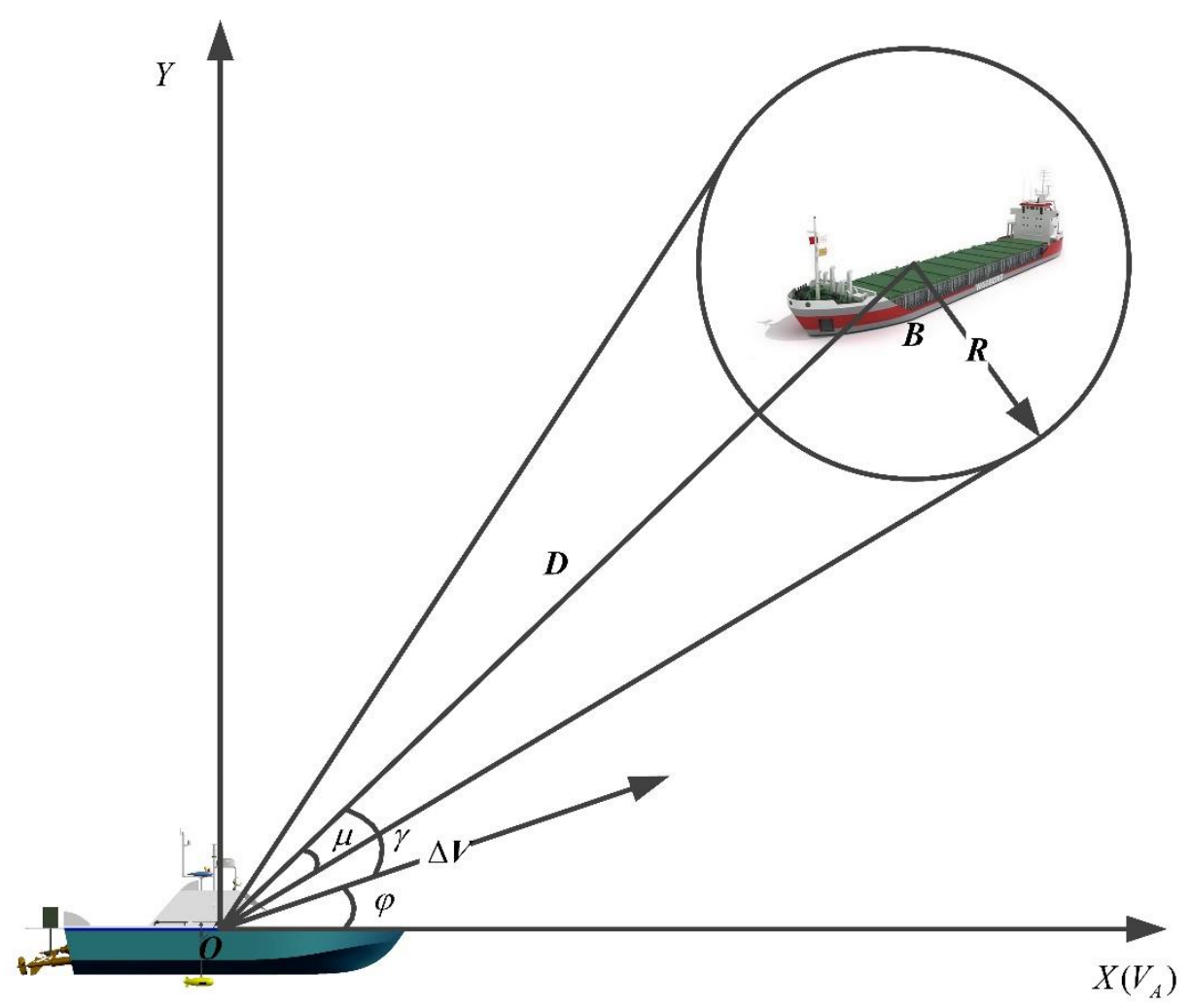

FIGURE 4. The relative coordinate

In Figure 4, the USV is the coordinate origin denoted by $O$ and the X-axis is defined by the direction of velocity $V_{A}$. The obstacle encountered by the USV is denoted by $B$. The distance between the USV and the obstacle is $D$, which can be measured by radar sensors. $R$ is the safe distance, and $\mu$ denotes the safe or collision angle. If angle $\gamma$ is larger than angle $\mu$, the USV can be considered as in a safe encountering situation; otherwise, possible collisions will occur. Such a collision assessment relationship can be represented as:

$$
\begin{cases}\gamma \leq \mu & \text { collision_flag }=1 \\ \gamma>\mu & \text { collision_flag }=0\end{cases}
$$

where collision_flag indicates whether a collision exists.

From Figure 4, the variation range of $\Delta \gamma$ is called the collision field, which is the angle change for $\Delta V$.

$$
\left\{\begin{array}{l}
\Delta \gamma=\mu-\gamma \\
\Delta \gamma=\mu+\gamma
\end{array}\right.
$$


The variations of the USV's velocity $\Delta V_{A}$ and heading $\Delta \alpha$ magnitude for each time interval can be obtained as follows:

$$
\left\{\begin{array}{l}
\Delta \alpha=\frac{\Delta \gamma\left[V_{A}-V_{B} \cos (\alpha-\beta)\right]}{V_{A}} \\
\Delta V_{A}=-\Delta \gamma V_{B} \sin (\alpha-\beta)
\end{array}\right.
$$

When the obstacle is static, $\Delta \alpha=\Delta \gamma$. The USV needs to adjust the heading outside the collision area.

\subsection{Navigating high-speed USVs with guaranteed safety}

Compared to operating USVs at low-speed or medium-speed, navigating a high-speed USV in a cluttered environment with obstacles is more challenging. Instability generated by high-speed motion is one of the main difficulties and in this section, by fully accommodating the features of high-speed USVs, a novel collision avoidance strategy has been designed and developed. The details of the problems associated with high-speed navigation will be first discussed and followed by the description of the designed collision avoidance algorithm.

\subsubsection{Problems with high-speed navigation}

When USVs are travelling at high speeds, following problems will occur and should be properly addressed.

\section{(1) The instability of obstacles.}

As the dominant sensors for environmental perception, marine radar and cameras detect and identify obstacles to construct a local environment map, which is updated in real-time. However, maritime environments associated with environmental aspects, such as waves, coasts or reefs, clouds, sky, mountains and other weather conditions including rain and snow, haze, sun light, are of high complexity and uncertainty making the accurate detection of obstacles difficult. For example, the interference to radar signals generated by surface waves can cause substantial noises in radar images making the detection of obstacles uncertain 
(Figure 5a). Such an uncertainty can also be observed in infrared and visible images (Figure 5b) caused by reflections from of sea surface. Under some extreme weather conditions such as fog, the contrast and clarity of infrared and visible images are largely constrained with a severe degradation in image resolution making the detection of obstacles (Figure 5c).

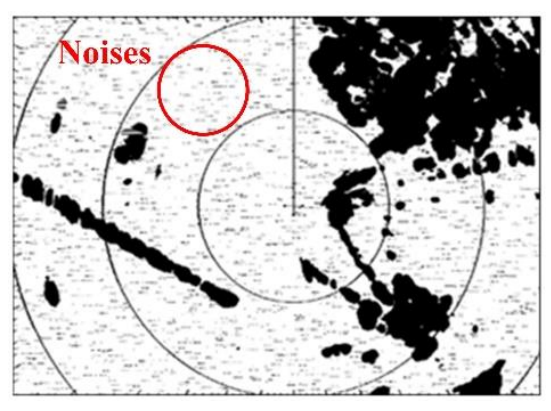

(a)

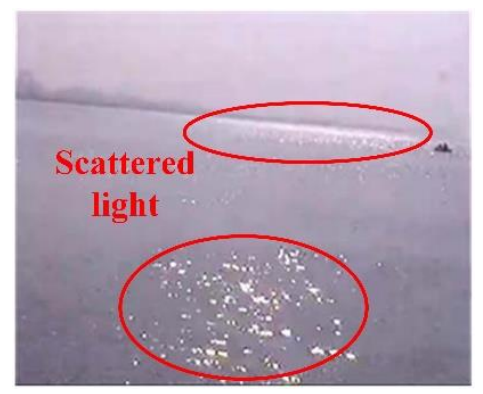

(b)

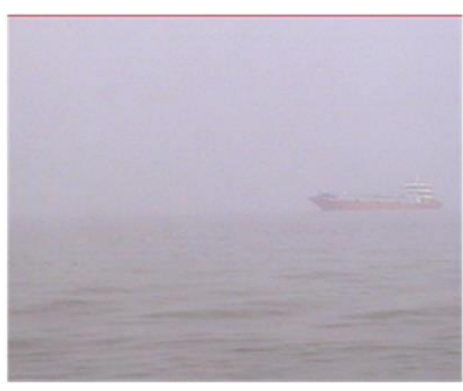

(c)

FIGURE 5 The influence of typical marine environment on environmental perception. (a) Clutter interference in radar image. (b) Scattered light interference in visible image. (c) Visible image in foggy weather

The performance of marine radar also affects the stability of obstacles, i.e. blind areas may be caused generating ambiguities for some obstacles. For example, the shape and brightness of the same obstacle may change in different frames with some obstacles in image sequence maybe even disappeared. In Figure 6a, a typical scenario with false obstacles in radar image is presented; whereas in Figure $6 b-d$, the sizes of obstacle 1 and obstacle 2 are different in every frame, and in frame 3 (Figure 6d) obstacle 2 even disappears. Such a problem is of particular importance to high-speed USVs, as the normal scanning period of a marine radar (the scan period of Raymarine E120 digital radars used in this paper is approximately $2.5 \mathrm{~s}$ ) is too long for highspeed USV, which will cause motion parameters of the obstacle to be discontinuous.

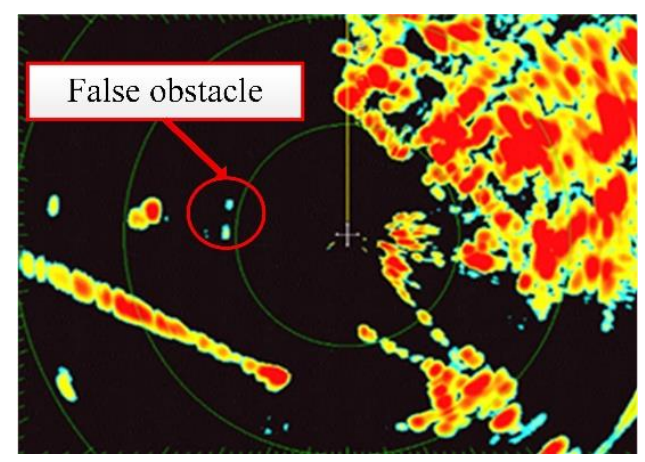

(a)

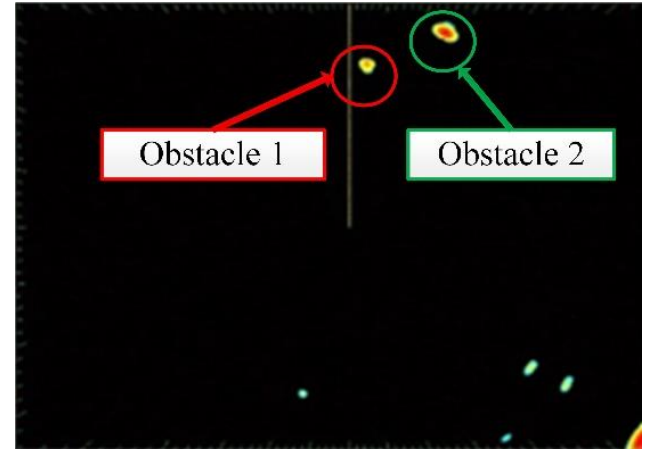

(b) 


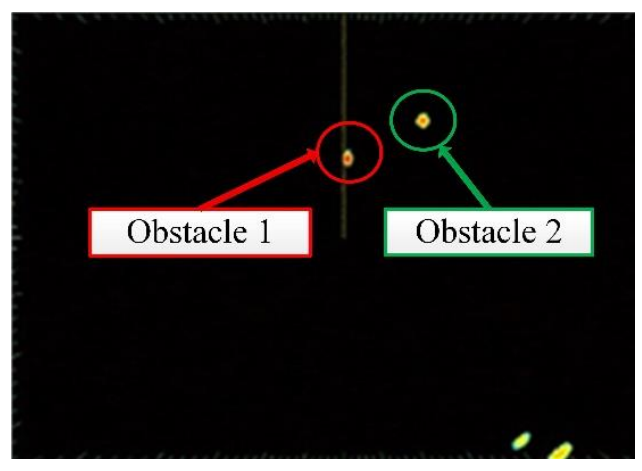

(c)

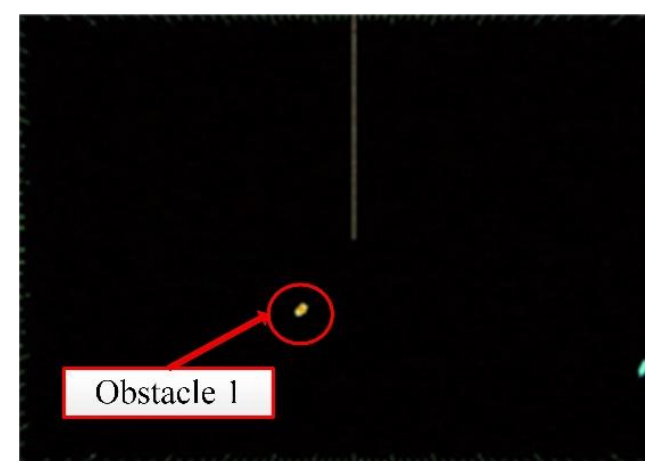

(d)

FIFURE 6 Typical radar image. (a) False obstacle in radar image. (b) Radar image sequence (frame 1). (c) Radar image sequence (frame 2). (d) Radar image sequence (frame 3)

\section{(2) The time lag and safety requirements in motion control}

High-speed USVs' motion dynamic characteristics is highly nonlinear and underactuated making it difficult to design a proper controller. In practice, the heading and speed control are normally adopted to achieve the motion state control. However, when USVs are travelling at a high speed, it becomes increasing challenging to make the vehicle complete the motion transition from the current state to the expected state without any time delay due to the reaction time required to actuate the propulsion system and propel the vehicle. As shown in Figure 7, the turning radius of high speed USV at 30 knots is about $106 \mathrm{~m}$, the turning rate is about $9 \%$.
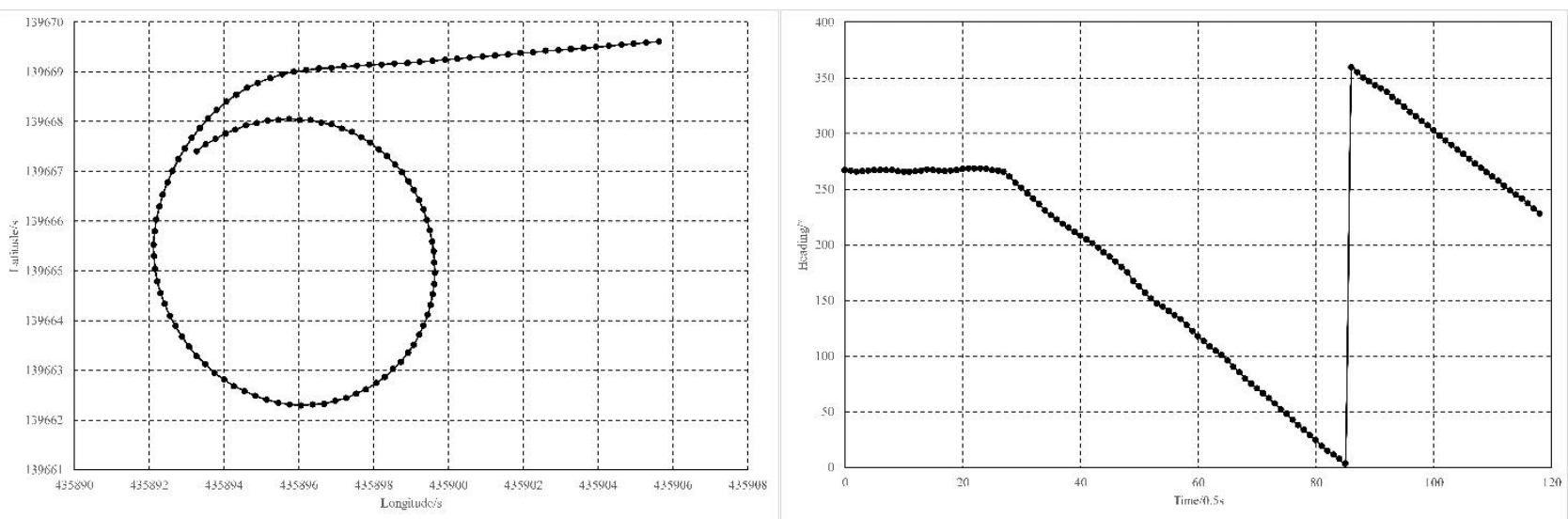

FIGURE 7 The track and heading of turning manoeuvrability test for high-speed USV at 30 knots 

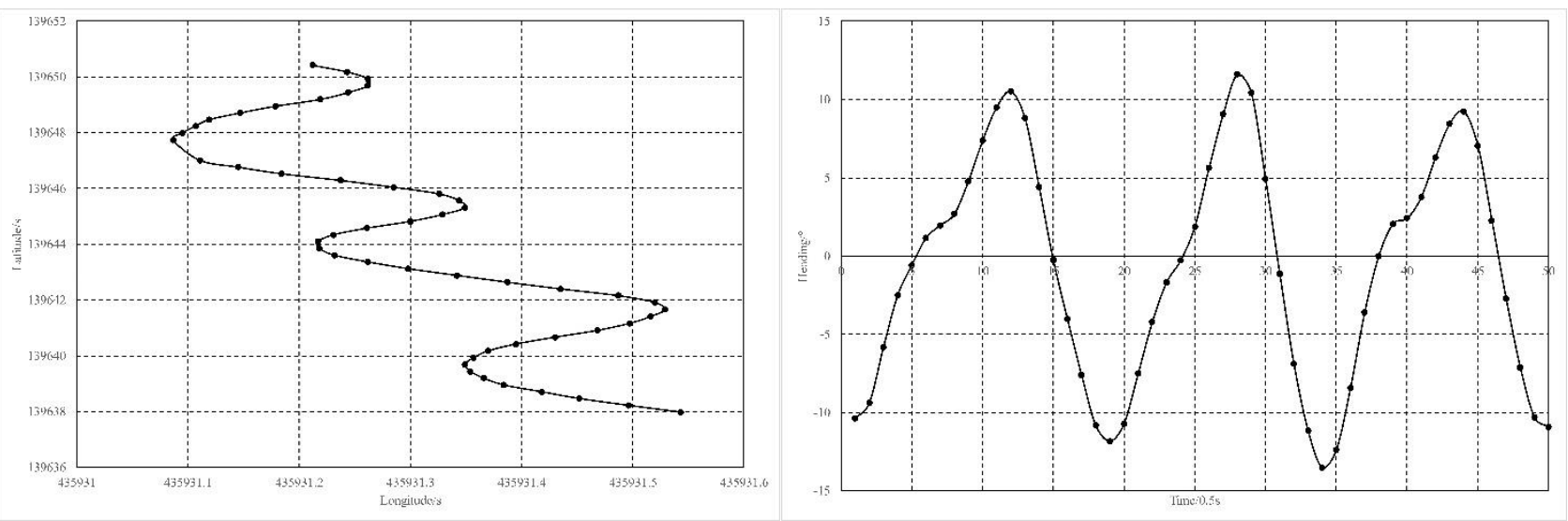

FIGURE 8 The track and heading of zigzag manoeuvrability test for high-speed USV at 30 knots

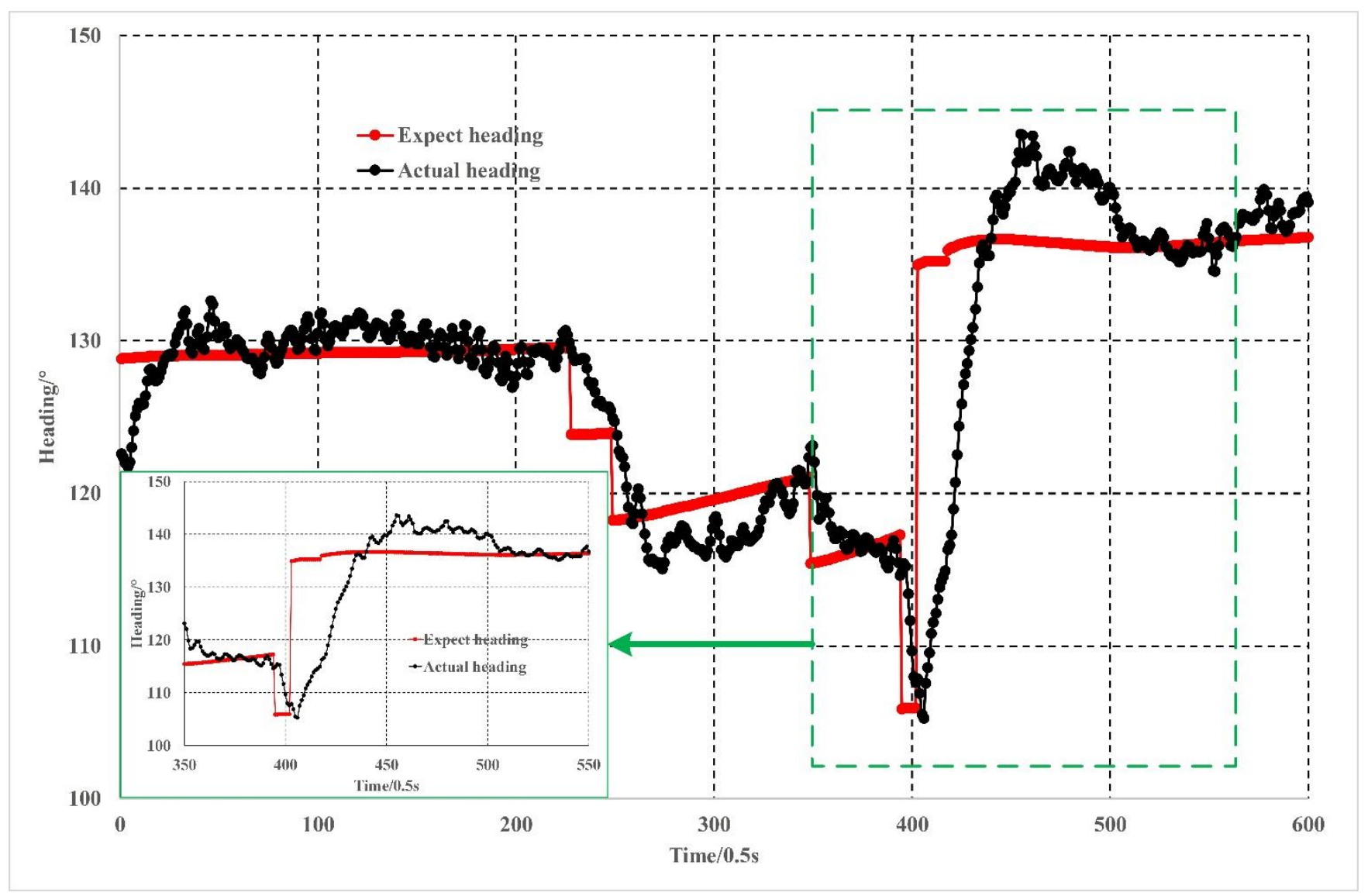

FIGURE 9 A delay of heading control for high-speed USV at 40 knots

In addition, the angular velocity of a high-speed USV is strictly constrained making the vehicle not able to execute a large angle steering in a short time. In particular, if the expected heading is in opposite direction to the current state, longer response time would be required. Such a problem can be further demonstrated by our previous test on a high-speed USV travelling at high speed. As shown in Figure 8, a turning cycle of heading from $10^{\circ}$ to $-10^{\circ}$ at 30 knots is about $7 \mathrm{~s}$. As shown in Figure 9, when the USV's speed is about 40 
knots, it would take approximately $20 \mathrm{~s}$ to complete a heading angle change from $105^{\circ}$ to $136^{\circ}$, and due to heading control overtake impact, the heading tends to be stable after another $30 \mathrm{~s}$. In some extreme situations, a repetitive large heading angle adjustment may lead to USV overturned or colliding with obstacles, which should be well addressed in practice.

\subsubsection{Reliable obstacle avoidance for high-speed USVs}

As mentioned previously, when USVs are sailing at high-speed, the incurred obstacle instability can influence the performance of collision avoidance, where a reliable evasive action is always required. In this paper, inspired from a number of different practical sea trials, a set of novel methods, including a stable local environment generation model and a robust obstacle avoidance method using velocity obstacle, have been proposed to specifically improve the reliability and stability of autonomous navigation systems of high-speed USV.

\section{(1) Stable local environment model generation}

In general, by taking the results of obstacle detection from an environmental perception system, a realtime local environment model can be constructed to describe the distribution of local obstacles. Obstacles are displayed as circles as shown in Figure 10 by fusing the information from vision systems and marine radar. The instability of obstacles also can be seen from Figure 10, i.e. Obstacle 1, 2 and 3 have different sizes and distributions between two time steps. 


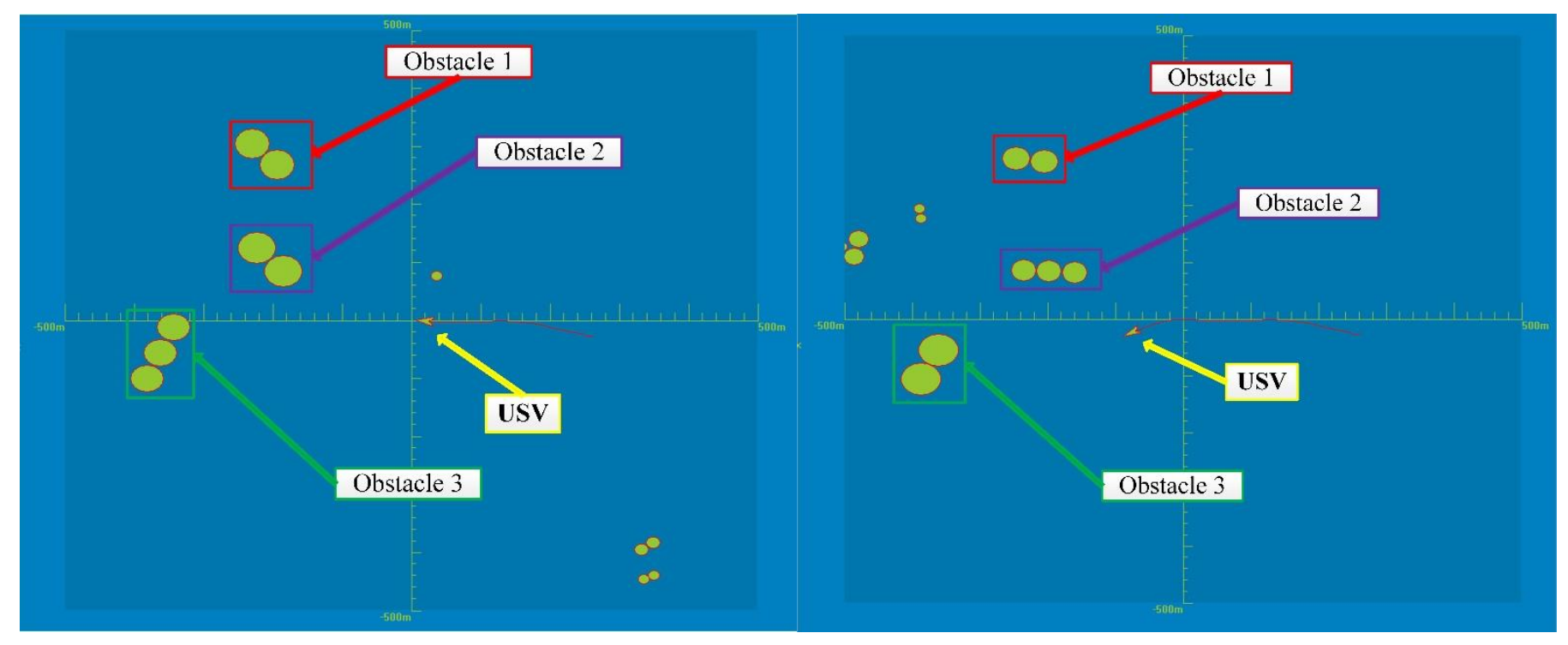

FIGURE 10 The results of environmental presentation

To solve the problem of obstacle instability, a new environment model generation algorithm that incrementally include obstacles into the model in discrete time domain has been proposed (shown in Fig. 11). To properly address the issue of 'false obstacle', an obstacle flagging process has been integrated into the algorithm to improve the accuracy of obstacle detection and subsequently increase the success rate of collision avoidance. Obstacles within the USV's vicinity (update area) are constantly monitored and compared with historical records. As shown in Figure 11, the more successful the matches with historical data are, the darker the colour of the obstacles. In this example, obstacle 3 has a higher reliability than obstacle 1 and obstacle 2 . A similarity determination method is used to make sure that only the obstacles with high level of certainty will be included into the environment model, which will help algorithms generate safe evasive actions. In addition, when obstacles come into the blind area, a relatively conservative strategy will be adopted by stopping obstacle information update to ensure these obstacles will not disappear (shown as the obstacle 4 in Figure 11). 


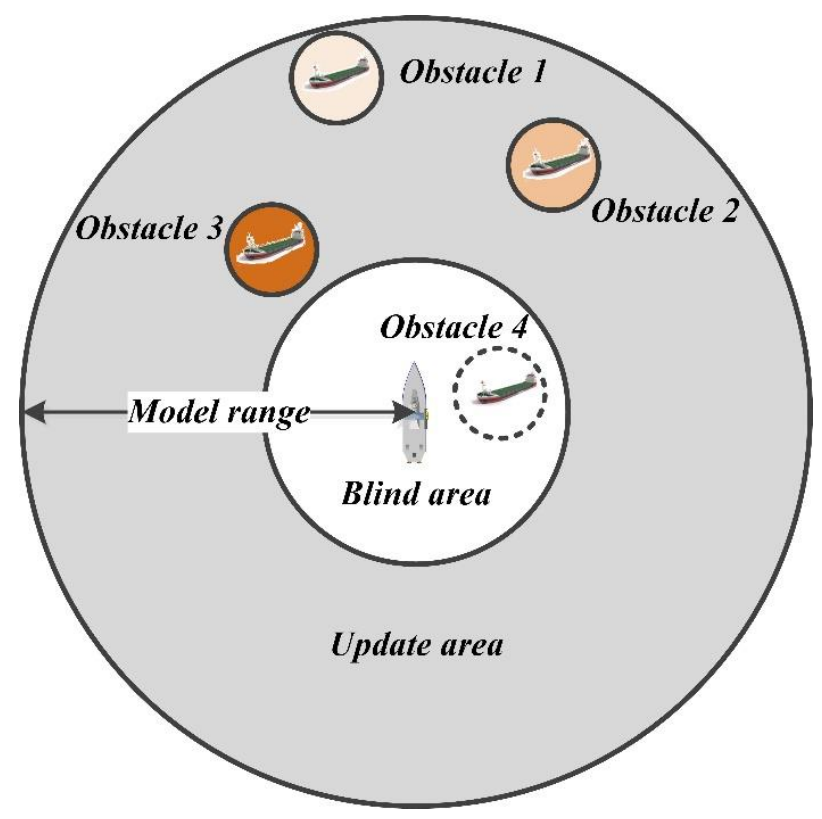

FIGURE 11 The results of environmental presentation

Details of the proposed algorithm is discussed as follows (algorithm flowchart is shown in Figure 12):

Step 1: Environment model initialization, with the number of obstacles in the model (obstacle_modle_num) is equal to 0 .

Step 2: Update the current time step, $t$, and set the environment model's centre to the USV's geographic coordinates (lon_ship, lat_ship), and the range, (obstacle_model_range) to the current radar range. obstacle_model_range can be calculated based on (4).

$$
\left\{\begin{array}{lc}
\text { obstacle_model_range }=1 \mathrm{n} \text { mile } & V_{A} \geq 40 \mathrm{kn} \\
\text { obstacle_model_range }=0.5 \mathrm{n} \text { mile } & 20 \mathrm{kn} \leq V_{A}<40 \mathrm{kn} \\
\text { obstacle_model_range }=0.25 \mathrm{n} \text { mile } & 0 \mathrm{kn} \leq V_{A}<20 \mathrm{kn}
\end{array}\right.
$$

Step 3: Determine whether all the obstacles (obstacle_model) are in the current model range; if the $j^{\text {th }}$ obstacle $\left(\right.$ obstacle $_{-}$model $\left._{j}\right)$ is outside the current model range, it is removed from the environment model and the number of obstacles in the model minus one.

Step 4: Using the USV's current latitude, longitude (lon_ship, lat_ship ) and heading $(\alpha)$, integrating obstacle's relative distance $(D)$, relative direction angle $(\theta)$, the $i^{\text {th }}$ obstacle's latitude and longitude $\left(l o n_{-} o b s_{i}, l a t_{-} o b s_{i}\right)$ can be calculated as: 


$$
\left\{\begin{array}{l}
l o n_{-} o b s_{i}=l o n_{-} \text {ship }+\frac{D \cdot \cos (\theta+\alpha)}{\varepsilon} \\
l a t_{-} o b s_{i}=l a t_{-} \text {ship }+\frac{D \cdot \sin (\theta+\alpha)}{\varepsilon \cdot \cos \left(l_{\text {lon }} \text { ship }\right)}
\end{array}\right.
$$

where $\varepsilon$ is the longitude circle distance between adjacent two seconds.

Step 5: Determine whether the $i^{\text {th }}$ obstacle $\left(o b s_{i}\right)$ is outside the updated distance $(d)$. If the $i^{\text {th }}$ obstacle is within the updated distance, go to step 7. $d$ is the blind of marine radar, based on sea trials, $d=200 \mathrm{~m}$.

Step 6: Determine whether $o b s_{i}$ is a new obstacle. Comparing the distance and radius of $o b s_{i}$ with all other obstacles within the current environment model. If the distance between $o b s_{i}$ and obstacle $_{-}$model $_{j}$ is less than the radius $\left(R_{i}\right)$ of $o b s_{i}$, and the difference between the two obstacles' radius is no more than

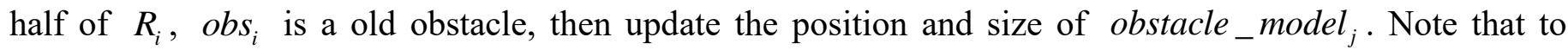
indicate the degree of stability of obstacles in the environment model, a new parameter names as obstacle model flag (obstacle_model_flag) is assigned, the value of obstacle model flag will increase with the number of successful matches. If no original environment models are close to $o b s_{i}, o b s_{i}$ is a new obstacle, and the number of obstacles in the model (obstacle_model_num) plus one.

Step 7: Repeat the same process of obstacle matching for the next obstacle and return to step 4 until all the obstacles have been assessed.

Step 8: Send the position and size information of the obstacle in environment model which satisfy the threshold $\xi$ to path plan system. $\xi$ can calculate based on as follows:

$$
\left\{\begin{array}{cc}
\xi=2 & V_{A} \geq 40 k n \\
\xi=3 & 20 k n \leq V_{A}<40 k n \\
\xi=4 & 0 k n \leq V_{A}<20 k n
\end{array}\right.
$$

Step 9: Enter the next time step environment model building cycle by returning to step 2. 


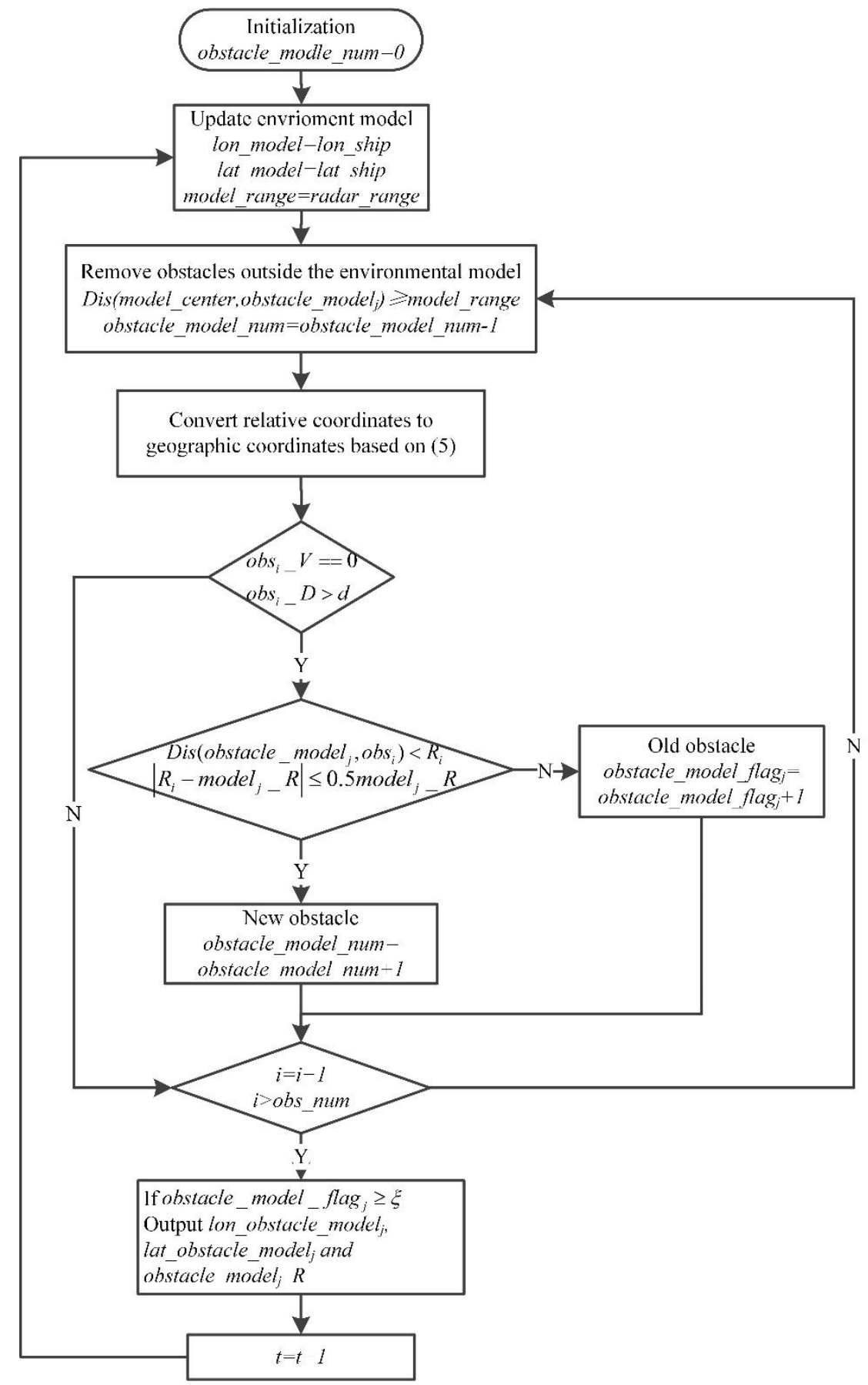

FIGURE 12 Flow chart of local environment model construction

\section{(2) Maintaining the stability of USV heading}

The velocity obstacle (VO) approach is used for guiding the USV to avoid obstacles. In the case of multiple obstacles, the VO approach is implemented in a way that the relative velocity is calculated with respect to each obstacle in the environment and the speed and heading of USV is adjusted according to the largest relative velocity.

The collision avoidance capability in this paper is achieved by adjusting heading angle of USVs. Such a 
strategy needs to take into account two critical aspects, i.e. COLGREs adherence in complex encountering situation and vehicle's dynamics constrained manoeuvre safety. More specifically, as a surface ship, it is important for USVs to maintain a compliance with COLREGs, especially in open-sea areas encountering with other ships. It is reasonable to suggest that when a moving obstacle is first detected, USV's heading will be adjusted according to COLREGs. However, for a high-speed USV with cruising speed over 40 knots, a constant adjustment of heading angles within a short control interval is difficult and dangerous. Therefore, when a high-speed USV requires to deal with collision avoidance with multiple moving ships, the navigation safety is all the more important and a steady and stable heading change is preferred. This paper therefore proposes a new heading determination approach to avoid any severe and unnecessary heading changes.

A typical situation consistent with the proposed strategy is shown in Figure 13, in which the dash line is the estimated track of a USV. During the initial stage, there are 3 obstacles need to be avoided. The moving obstacle 1 and the moving obstacle 2 both form the Crossing encounter situation, and to avoid the moving obstacle 1, a larger heading change is required according to COLREGs and VO approach. So, in this case the moving obstacle 1 is chosen as the reference obstacle, and the USV make a starboard action. As the USV continues sailing, the moving obstacle 3 enters the range of avoidance distance again forming a Crossing encounter situation. In such a circumstance, the USV is still in collision avoidance state, but the safety priority of USV is higher than COLREGs at this time, so the USV choses to continue to turn to its right instead of turning left according to COLREGs. 


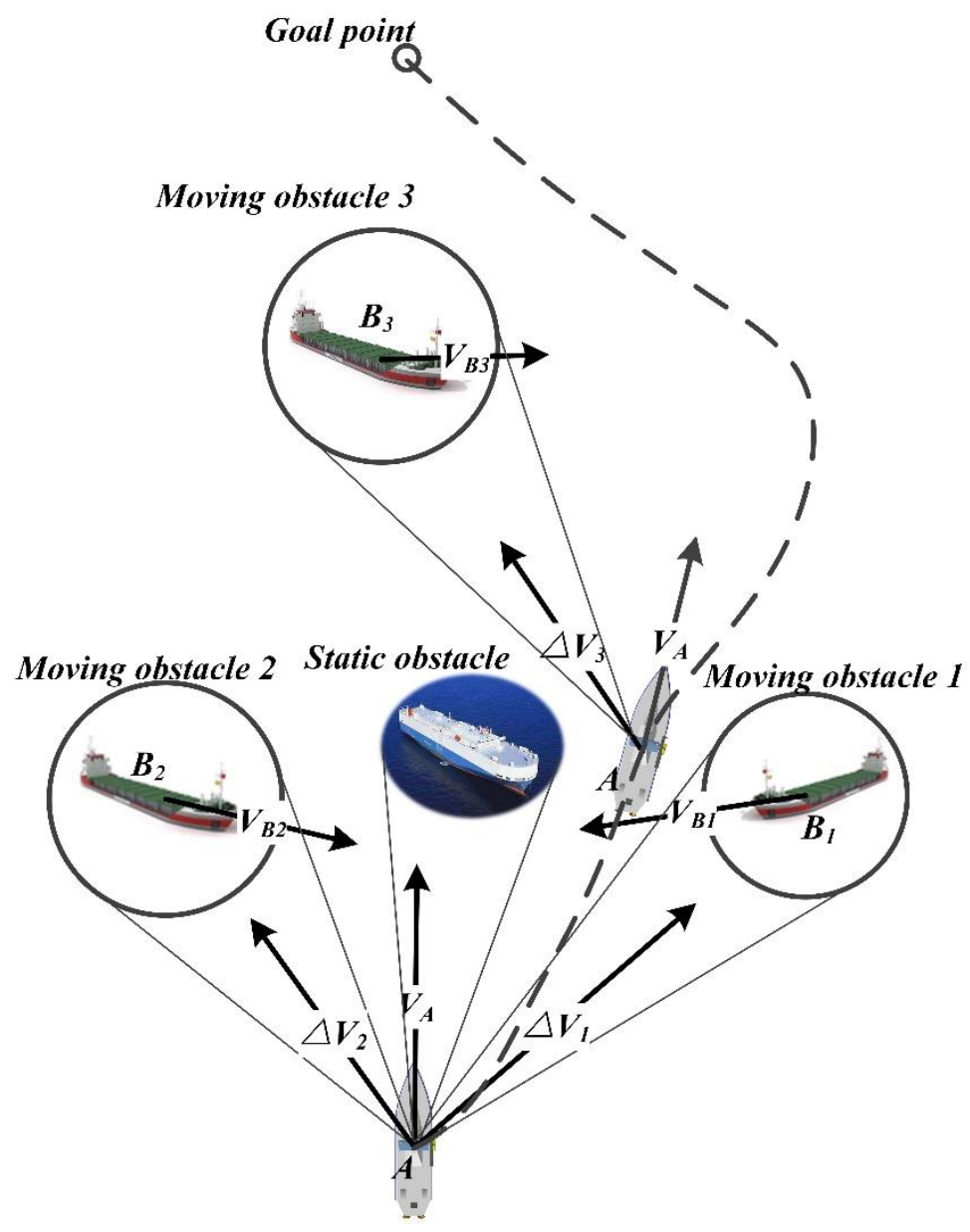

FIGURE 13 A typical situation for obstacle avoidance method

The detail of the algorithm is discussed as follows with the algorithm flow chart shown in Figure 14.

Step 1: Assign the USV's current speed $\left(V_{A}\right)$ and heading $(\alpha)$ to the expected $V_{A_{-} \text {expect }}=V_{A}$ and heading $\alpha_{\text {expect }}=\alpha$. Initialise the value of the number of obstacles and each obstacle's speed and heading according to sensor detection information.

Step 2: For each time step $t$, update value of $V_{A}$ and $\alpha$ according to IMU and GPS information.

Step 3: Calculate the heading towards the goal point $\left(\alpha_{\text {goal }}\right)$ using equation:

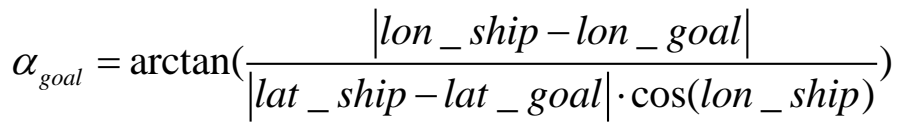

where (lat_ship, lon_ship) and (lat_goal, lon _ goal )) are the latitude and longitude of USV and goal point.

Step 4: Determine whether the $\alpha_{\text {goal }}$ collides with all obstacles using the collision assessment strategy shown in Eq. (1). If no collisions exist (collision_flag=0), go to Step 5. 
Step 5: Reset the value of turn_flag to 0 and the expect heading as $\alpha_{\text {expect }}=\alpha_{\text {goal }}$, go to Step 13. Note that the parameter turn_flag is assigned to record the historical turning behaviour of the USV, and only the evasive actions with the same turn_flag will be implemented to avoid any large heading angle change and stabilise a USV's to the most extent.

Step 6: If there are moving obstacles, calculate the relative velocity $\Delta V_{j}$ (based on $V_{A_{-} \text {expect }}, \alpha_{\text {expect }}$, $V_{B j}$, and $\beta_{j}$ ) for the $j^{\text {th }}$ moving obstacle $\left(M o v e_{-} o b s_{j}\right)$, or else go to Step 13.

Stpe 7: Determine whether a collision to Move_obs $_{j}$ exists and jump to Step 11 if there is no collisions. Note that to avoid any false alarm caused by uncertain obstacles, a new parameter names as avoidance distance (Avoid_dis) is introduced and only the obstacles staying inside avoidance distance will be assessed. The value of avoidance distance is configured according to USV's speed as:

$$
\left\{\begin{array}{lc}
\text { Avoid_dis }=0.5 n \text { mile } & V_{A} \geq 40 \mathrm{kn} \\
\text { Avoid_dis }=0.25 n \text { mile } & 20 k n \leq V_{A}<40 k n \\
\text { Avoid_dis }=0.15 n \text { mile } & 0 k n \leq V_{A}<20 k n
\end{array}\right.
$$

Step 8: If there is a historical record of turn_flag, go to Step 10.

Step 9: If turn_flag does not exist, it can be calculated based on COLREGs as:

$$
\begin{cases}\text { turn_flag }=1 & \text { If a right turn been generated } \\ \text { turn_flag }=2 & \text { If a left turn been generated }\end{cases}
$$

Step 10: Calculate the changes of the speed $\left(\Delta V_{A j}\right)$ and the heading $\left(\Delta \alpha_{j}\right)$, for the Move_obs $s_{j}$ using (3) based on turn_flag.

Step 11: Repeat the same process of collision assessment and velocity determination for the next moving obstacle and return to step 6 until all the moving obstacles have been assessed.

Step 12: Choose the obstacle with the largest heading change $\left(O b s_{\max }\right)$ as the reference obstacle and calculate the expected speed $V_{A_{-} \text {expect }}$ and heading $\alpha_{\text {expect }}$ using the equation as:

$$
\left\{\begin{array}{l}
V_{A_{-} \text {expect }}=V_{A_{-} \text {expect }}+\Delta V_{A \max } \\
\alpha_{\text {expect }}=\alpha_{\text {expect }}+\Delta \alpha_{\max }
\end{array}\right.
$$

Step 13: Determine whether a collision to static obstacle $O b s_{i}$ exists by using and jump to Step 17 if there is no collisions. 
Step 14: If there is a historical record of turn_flag, go to Step 16.

Step 15: If turn_flag does not exist, it can be calculated as (9).

Step 16: Calculate the changes of the heading $\left(\Delta \alpha_{i}\right)$, for the $i^{\text {th }} O b s_{i}$ using (3) as well as turn flag parameter.

Step 17: Repeat the same process of collision assessment and velocity determination for the next obstacle and return to step 6 until all the static obstacles have been assessed.

Step 18: Choose the obstacle with the largest heading change $\left(O b s_{\max }\right)$ as the reference obstacle and calculate the expected heading $\alpha_{\text {expect }}$ using (10).

Step 19: Send the control command ( $V_{A_{-} \text {expect }}$ and $\left.\alpha_{\text {expect }}\right)$ to the contorl system and enter the next cycle by returning to Step 2 .

It can be seen that with the introduction of the parameter turn flag, which records the historical evasive actions, the conventional VO approach has been modified and improved to be capable of operating high-speed USVs. Consistent turning actions can be ensured for collision avoidance to retain the stability of USVs. 


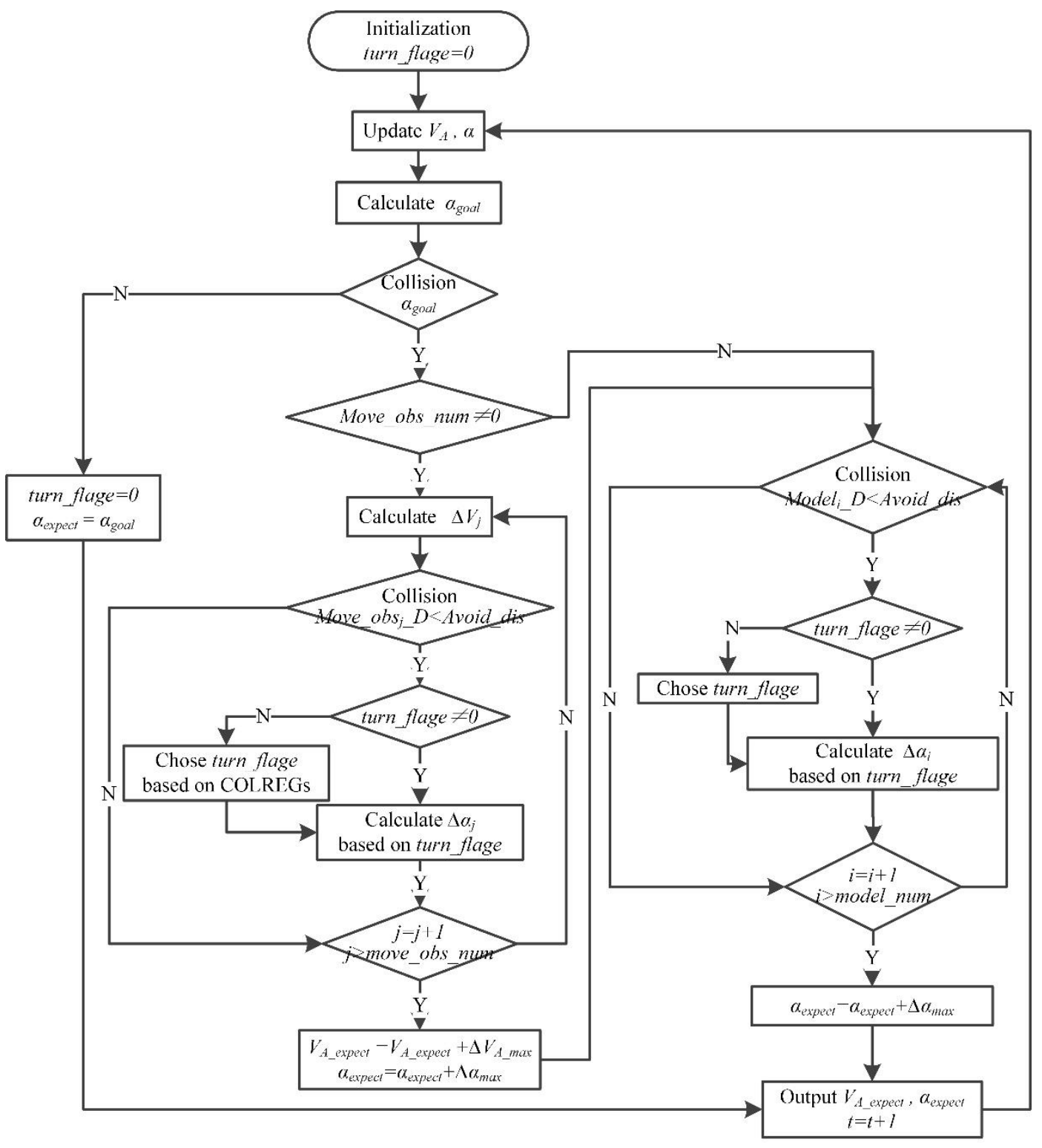

FIGURE 14 Flow chart of obstacle avoidance method

\section{Hardware-in-the-loop simulation test results and analyses}

To demonstrate the effectiveness of the proposed collision avoidance strategies, hardware-in-the-loop simulations of USV collision avoidance tests are provided in this section. Hardware-in-the-loop simulations can validate the ability of each sub-system for a long time in a convenient and cost-effective way. On the basis of verifying system's software logical structure, critical components such as the hardware system, data interface, and reliability of the system can also be well tested.

\subsection{Hardware-in-the-loop simulation system}

The general structure of the hardware-in-the-loop simulation system is shown in Figure 15. The system 
can be divided two subsystems: physical simulation subsystem and virtual simulation subsystem. The physical simulation subsystem contains three computers: monitor computer, PC104 embedded control computer and IPC visual computer. The virtual simulation subsystem contains two PC (visual simulation computer and sensor simulation computer) and one workstation. The software of hardware-in-the-loop simulation system is VC++ and Vega Prime.

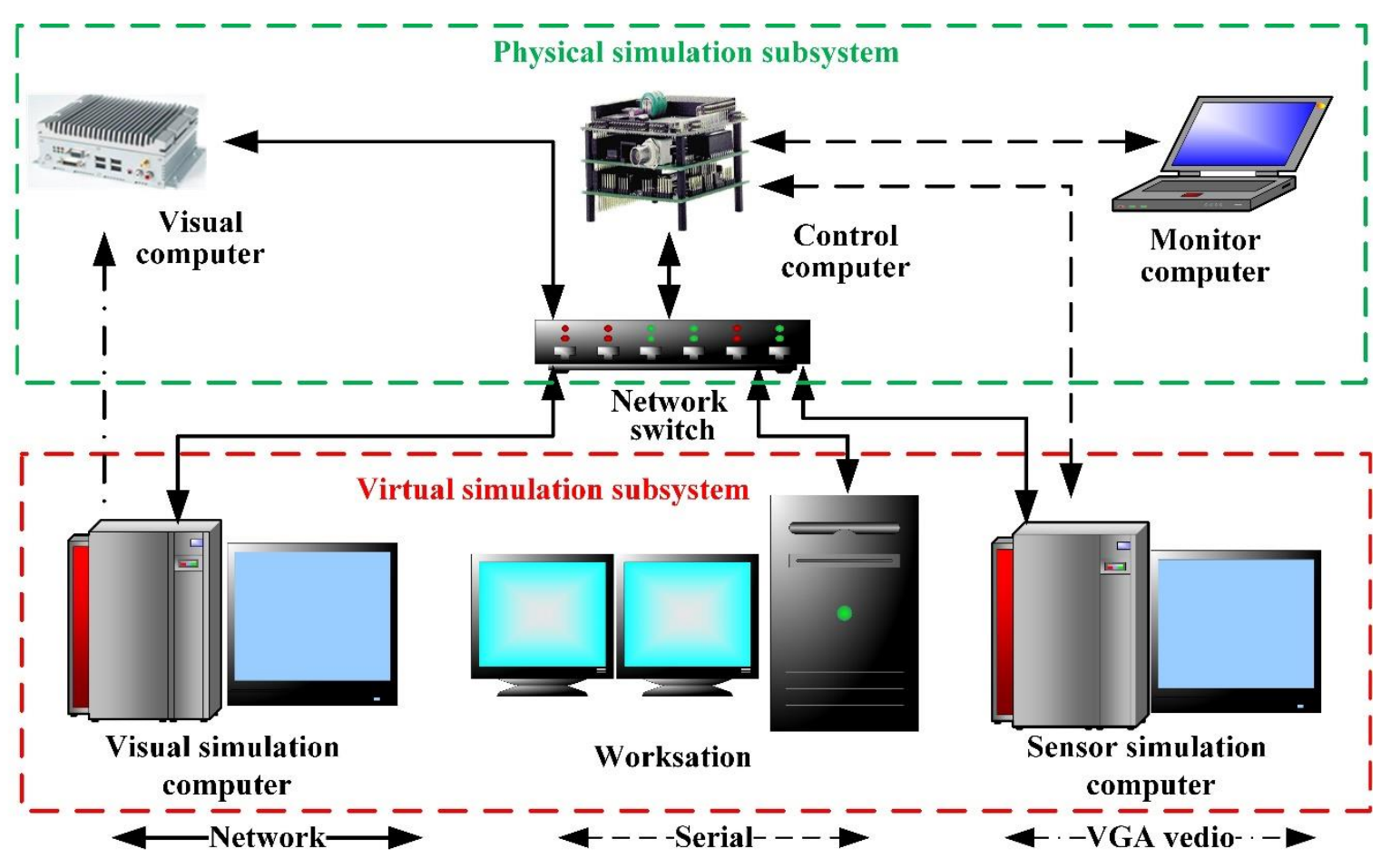

FIGURE 15 Hardware-in-the-loop simulation system general structure

The data flow loop of hardware-in-the-loop simulation test consists of the following parts:

(1) Workstation is the core of virtual simulation subsystem and is responsible for most of the simulation work. The simulation software running on it takes the output of the control system as the input, continuously updates the current state of USVs by solving the dynamic equation of USV and transforms the corresponding visual information according to a certain interface protocol. The simulation result is shown in Fig. 16 a.

(2) According to the obstacle position information provided by the workstation, the visual simulation computer can draw a simulated video image and output the VGA video stream to the IPC visual computer. According the environment and current state of USV provided by the workstation, the sensor simulation computer simulates the information of GPS, IMU, weather station and output sensor data to PC104 control computer using serial communication. 
(3) The visual computer calculates the size, location and speed of the obstacles based on the video from workstation and sends the information to the control computer by network communication. The simulation result is shown in Fig. 16 b.

(4) The control computer calculates the USV command information (expect heading, speed) through the collision avoidance algorithm, and the control algorithm solves the basic control commands (expected rudder angle, engine throttle) and returns them to the workstation by network communication.

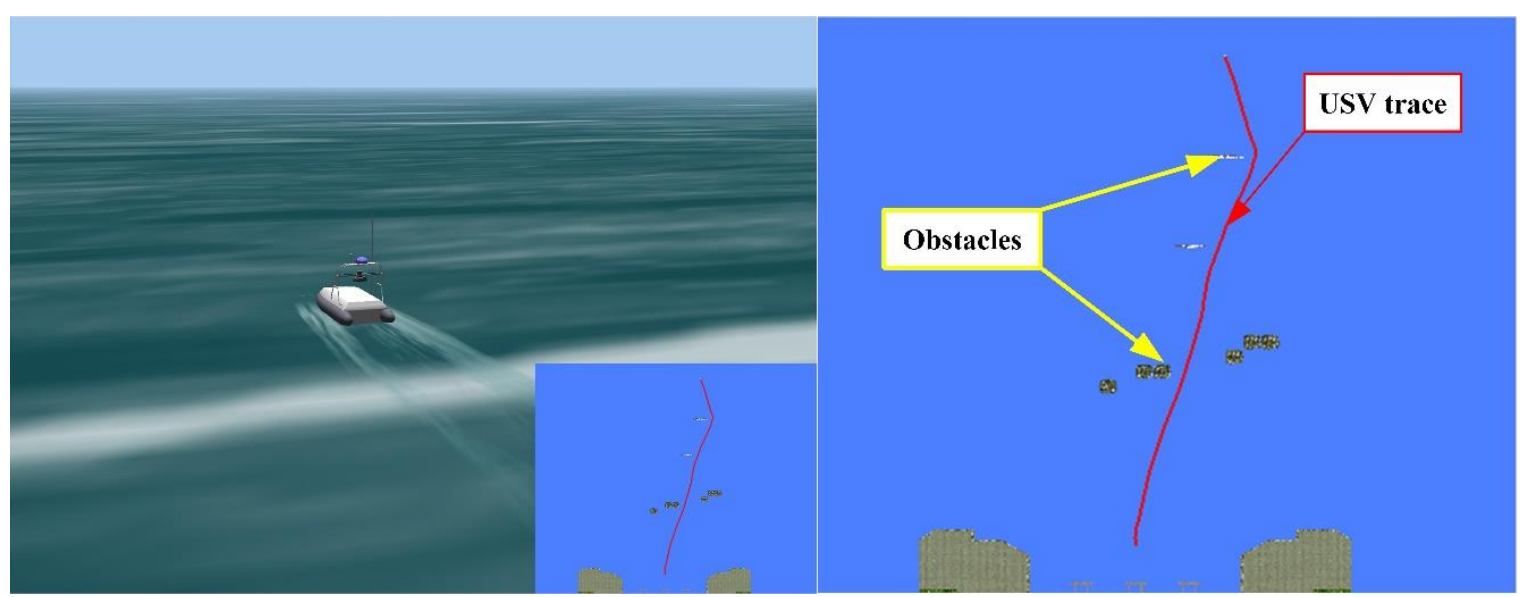

(a)

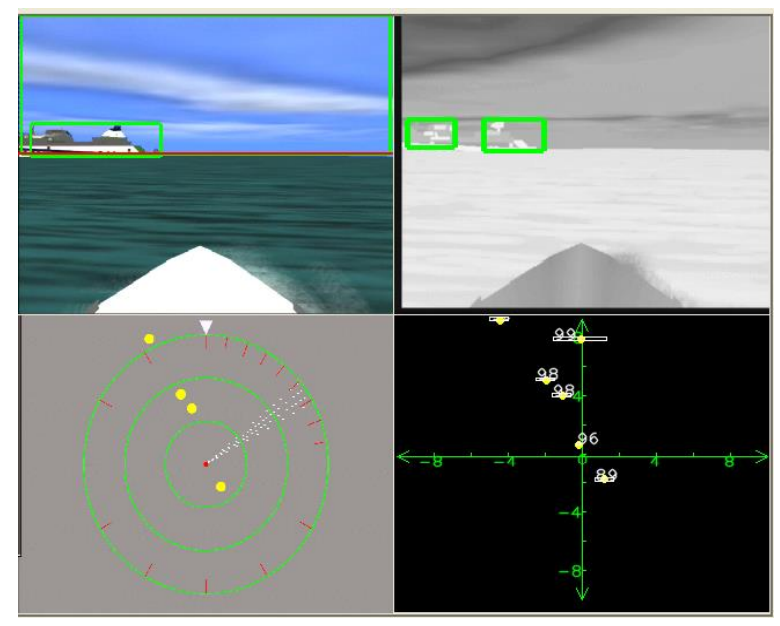

(b)

FIGURE 16 The hardware-in-the-loop simulation results. (a) The simulation result on the workstation. (b) The simulation result on the visual computer

\subsection{Multiple obstacles collision avoidance simulation tests}

\subsubsection{Static obstacles collision avoidance simulation test}

The multiple static obstacles avoidance region was designed to verify the capacity of the proposed collision avoidance strategies. As shown in Figure 17, the test was designed to let the USV travel from south 
to north. There are two single obstacles and two arrays of obstacles on the route. The speed of USV is 40knots.

Detailed collision avoidance process is shown in Figure 17 with the variations of heading of the USV represented in Figure 18. As shown in Figure 18, four evasive actions were taken by the USV. A stable control command (the expected heading angle shown as the red line in Figure 18) can always be generated to ensure that a consistent evasive action is implemented. Note that such a strategy is critical for high-speed USVs encountering with cluttered obstacles as any inconsistency in manoeuvre would lead to unexpected collisions.

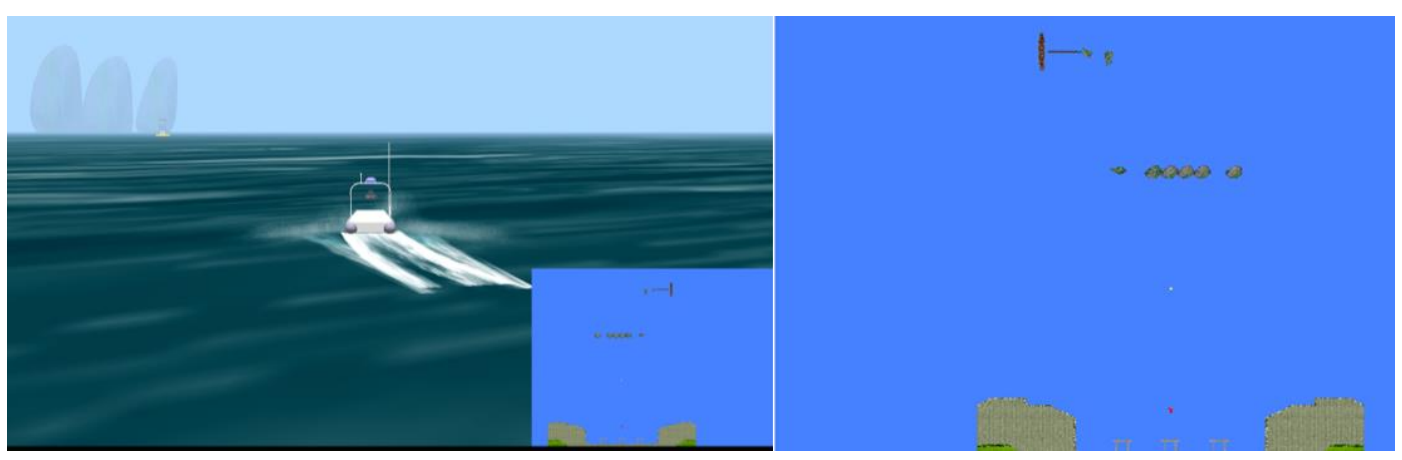

(a) Time step $=0$

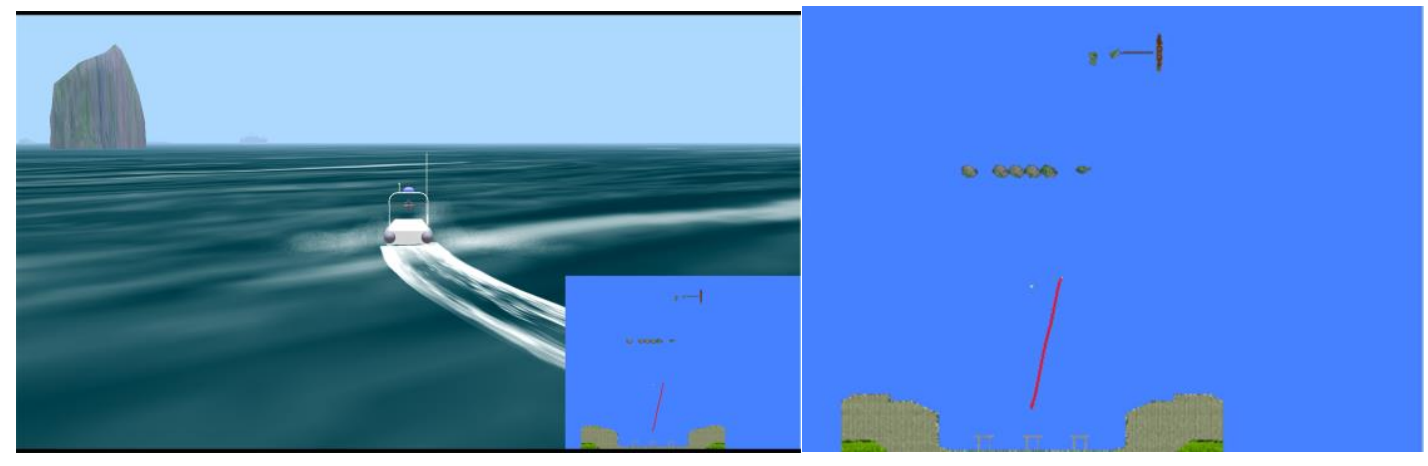

(b) Time step $=62$

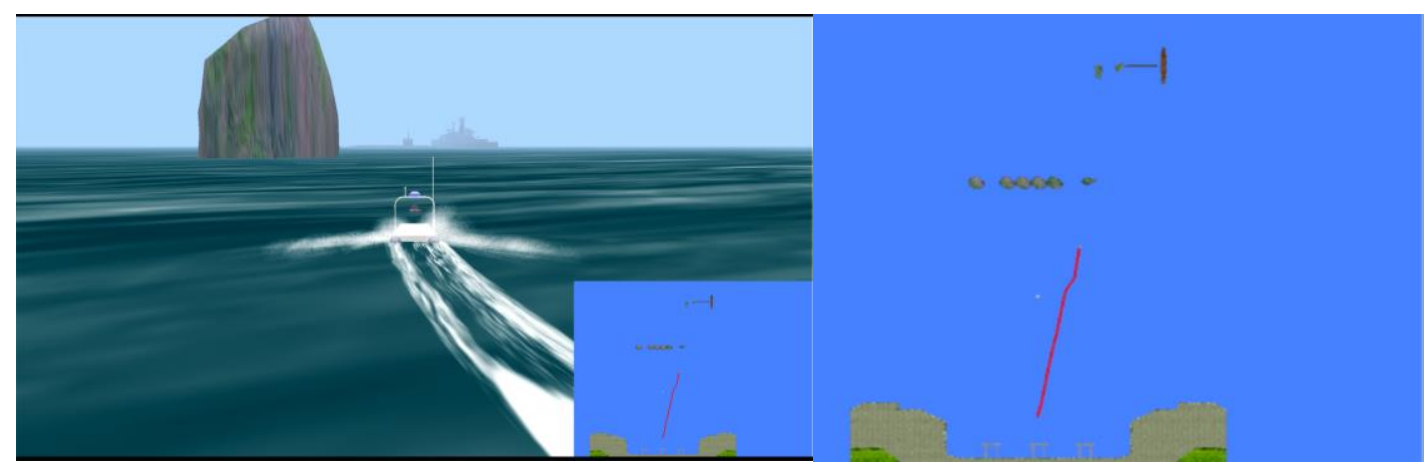

(c) Time step $=103$

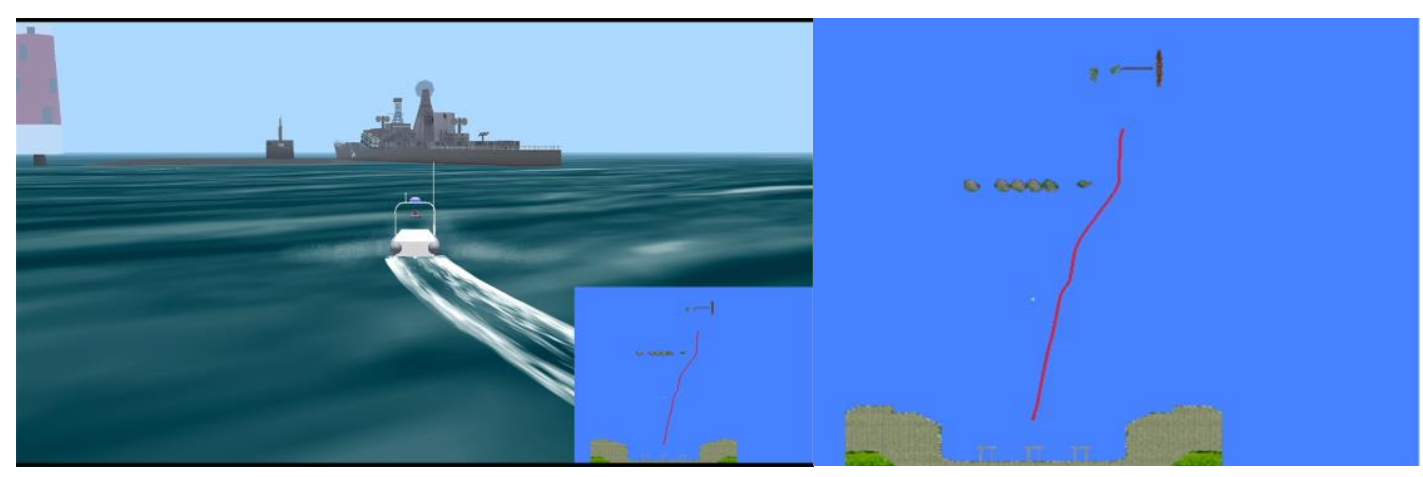

(d) Time step $=181$ 


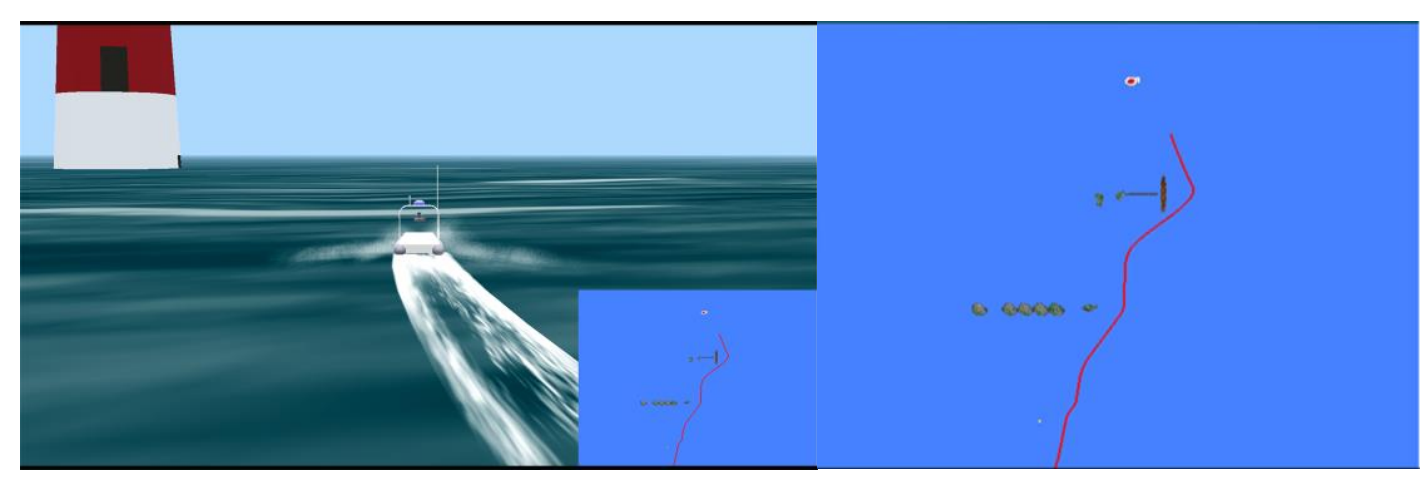

(e) Time step $=290$

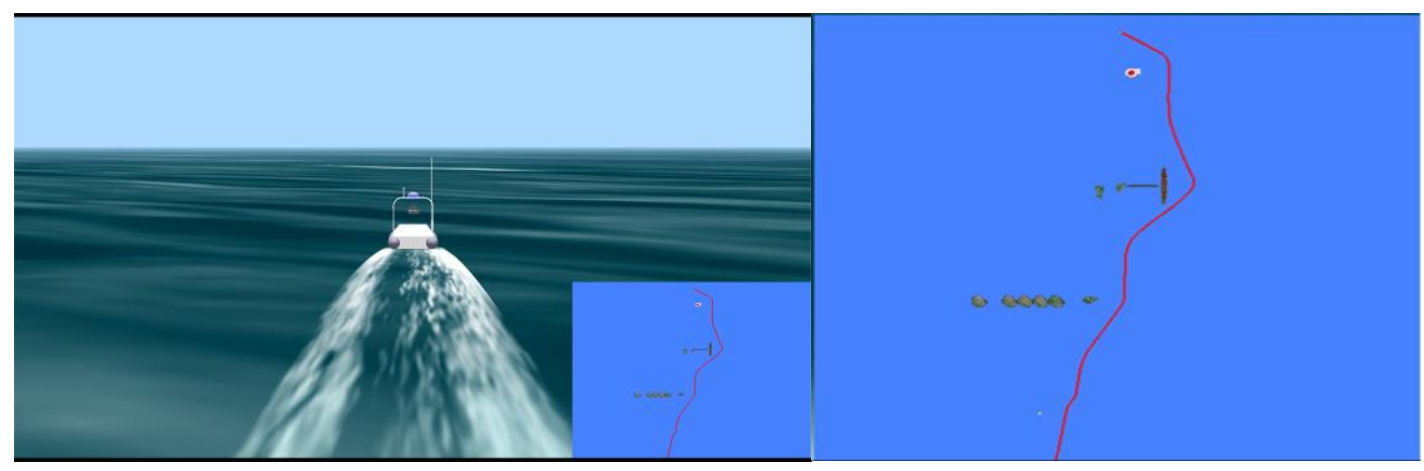

(f) Time step=371

FIGURE 17 The process of multi static obstacles collision avoidance simulation test. (a) Time step=0. (b) Time step=62. (c) Time step=103. (d) Time step=181. (e) Time step=290. (f) Time step=371

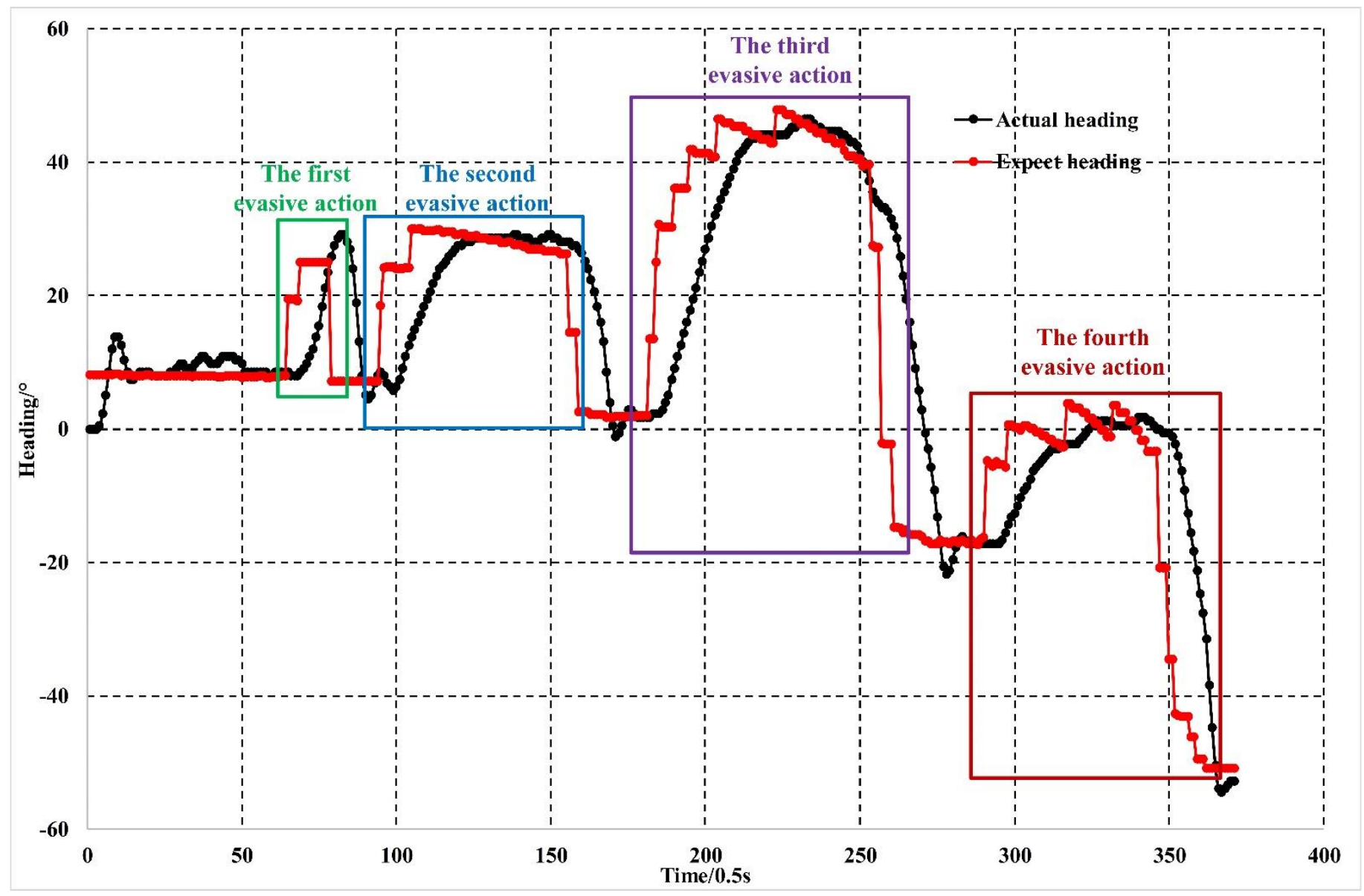

FIGURE 18 The heading of multi static obstacles collision avoidance simulation test

\subsubsection{Moving obstacles collision avoidance simulation test}


A further test was designed to evaluate the COLREGs compliant collision avoidance capability for highspeed USVs. As shown in Figure 19, four static obstacles and one moving ship are added into the simulation scenario. With the USV travelling from south to north and the moving ship travelling from east to west, a Crossing encounter situation is formed. The speed of USV is 40knots and the speed of moving ship is $20 \mathrm{knots}$. The dashed line is the trace of moving ship. The trace of USV is shown as the red lines in Figure 19, which clearly demonstrates that the USV can successfully avoid moving obstacles complied with the COLREGs while navigating.

The detailed avoidance process is shown in Figure 19 with the variations of heading shown in Figure 20. In Figure 20, during initial stages, the expect heading $\alpha_{\text {expect }}$ was about $0^{\circ}$ and the USV was travelling to the goal point at 40 knots speed. At time step 171, the moving ship entered the avoidance distance Avoid_dis, USV accordingly made a port manoeuvre to avoid the moving obstacle with the $\alpha_{\text {expect }}$ adjusted to $-33.8^{\circ}$ (as shown in Figure 19b). During the obstacle avoidance process, the $\alpha_{\text {expect }}$ was stable at $-33.8^{\circ}$. After time step 196, the USV successfully passed abaft of (behind) the moving obstacle which was complied with the COLREGs. Then the static obstacles entered the Avoid_dis, the heading was adjusted to make a left turning to $-42.3^{\circ}$, after time step $239 \alpha_{\text {expect }}$ was changed back to $\alpha_{\text {goal }}$.

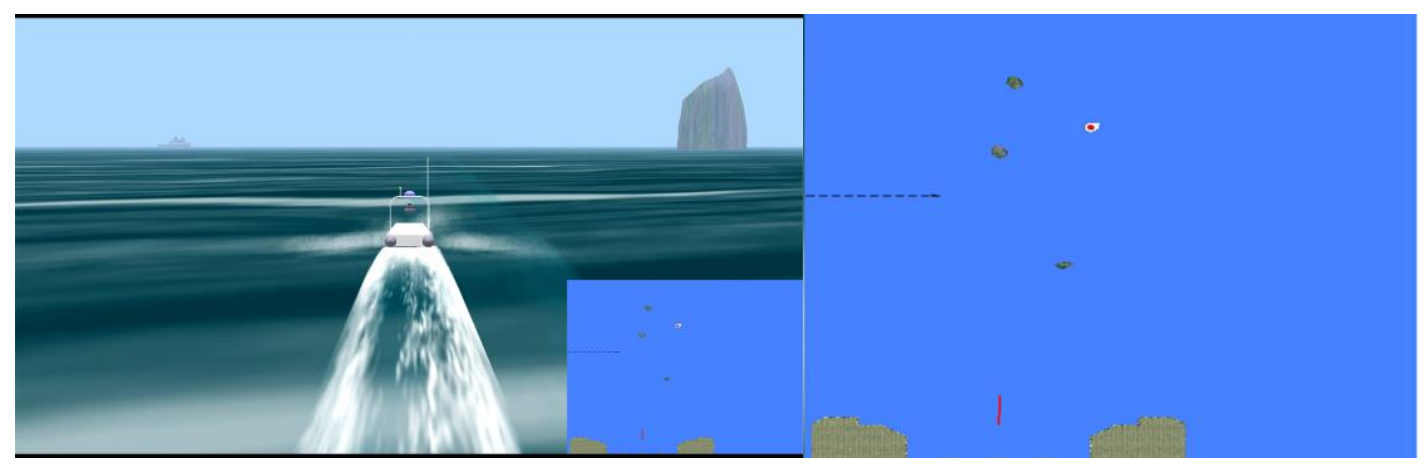

(a)

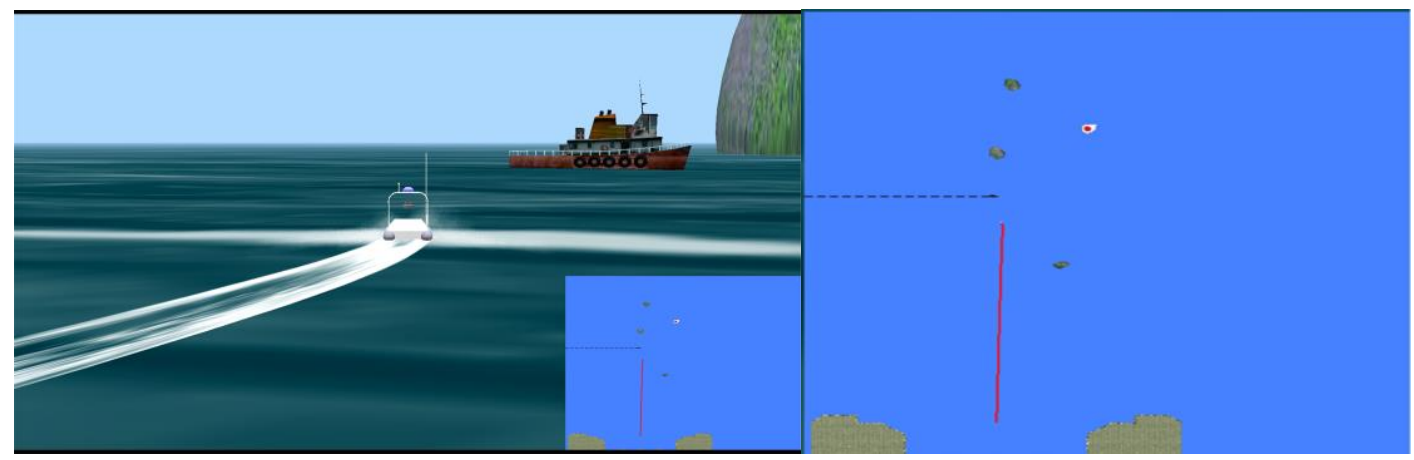

(b) 


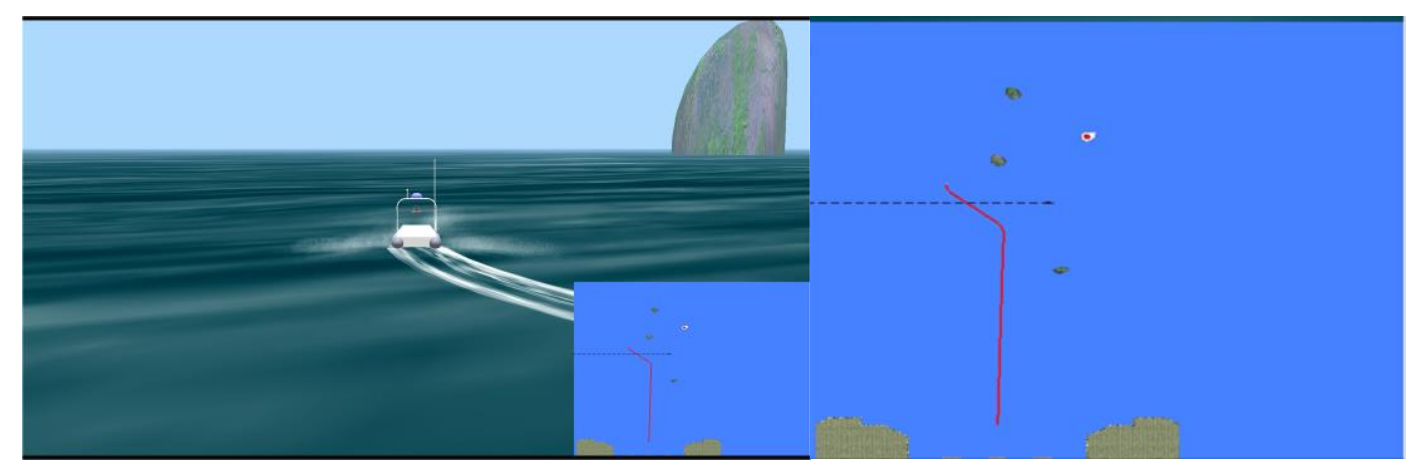

(c)

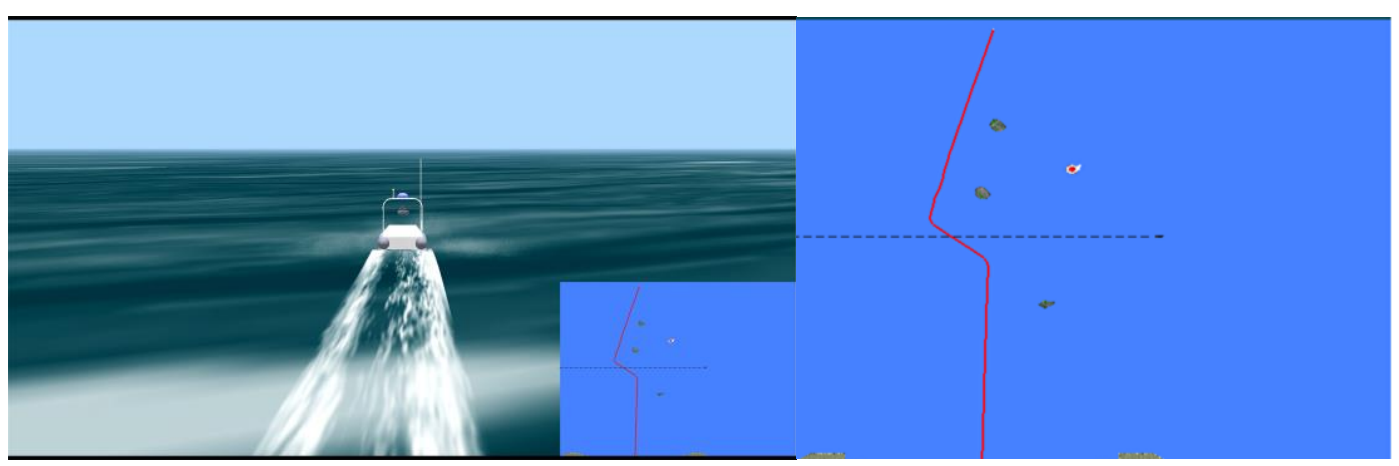

(d)

FIGURE 19 The process of moving obstacles collision avoidance simulation test. (a) Time step=10. (b) Time step=171. (c)

Time step=239. $(\mathrm{d})$ Time $\mathrm{step}=400$

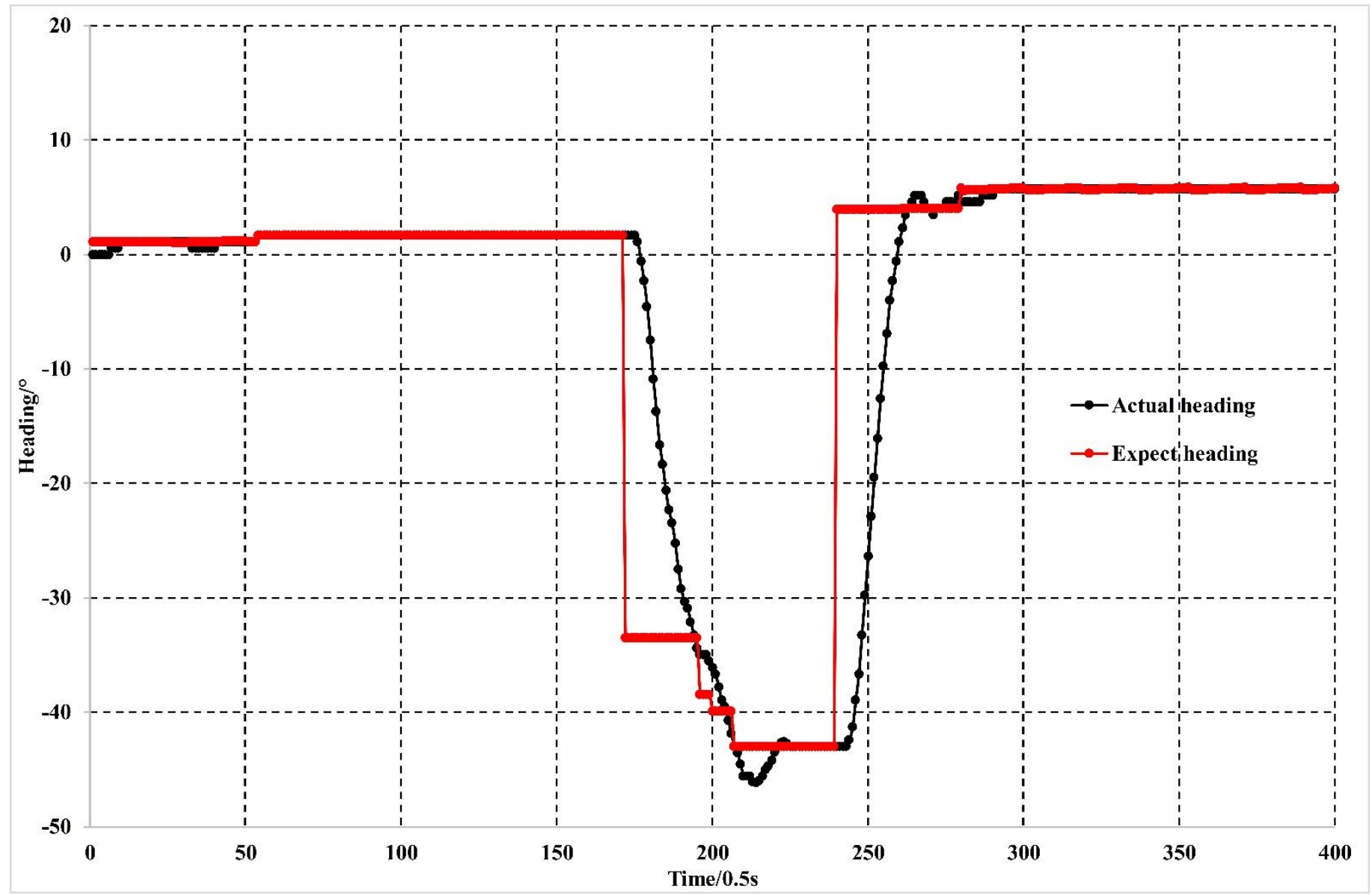

FIGURE 20 The heading of moving obstacles collision avoidance simulation test 


\section{High-speed USVs sea trials results and analyses}

To verify and validate the proposed autonomous navigation system as well as the collision avoidance strategies, a set of sea trials have been carried out in various open sea areas in China. The trials require a coordinated functioning of key components within the autonomous navigation system and a smooth information communication was established within subsystems of environment perception, path planning, and motion control. With the primary aim of sea trials being the verification of the obstacle detection and collision avoidance capabilities, three different types of trials were undertaken with various testing configures as: 1) sea trials in a constrained sea area with sparse obstacles, 2) sea trials in a harbour area with dense obstacles and 3) sea trials in an open sea area with high speed (40 knots).

\subsection{Main parameters of 'Tianxing No.1' USV}

'Tianxing No.1' USV was selected as the testing platform with its main parameters shown in Table 2. Figure 21 illustrates some key moments during the trials with Figure 21 a displaying the snapshot of 'Tianxing No.1' navigating on an open sea and Figure 21b showing how 'Tianxing No.1' taking actions to avoid three anchored ships.

TABLE 2 Main parameters of the 'Tianxing No.1' USV

\begin{tabular}{c|c}
\hline Total length & $12.2 \mathrm{~m}$ \\
Displacement & $7 \mathrm{t}$ \\
Maximum speed & $50 \mathrm{knots}$ \\
Maximum range & $1000 \mathrm{~km}$ \\
Power and propulsion & Diesel engine + surface-drive and electronic propeller \\
Sensors & Electro-optical pod, marine radar, panoramic vision camera, multi- \\
& beam sonar, CTD, weather station etc. \\
Missions & Hydrology and meteorological information collection, ocean \\
& mapping, environmental observation \\
\hline
\end{tabular}




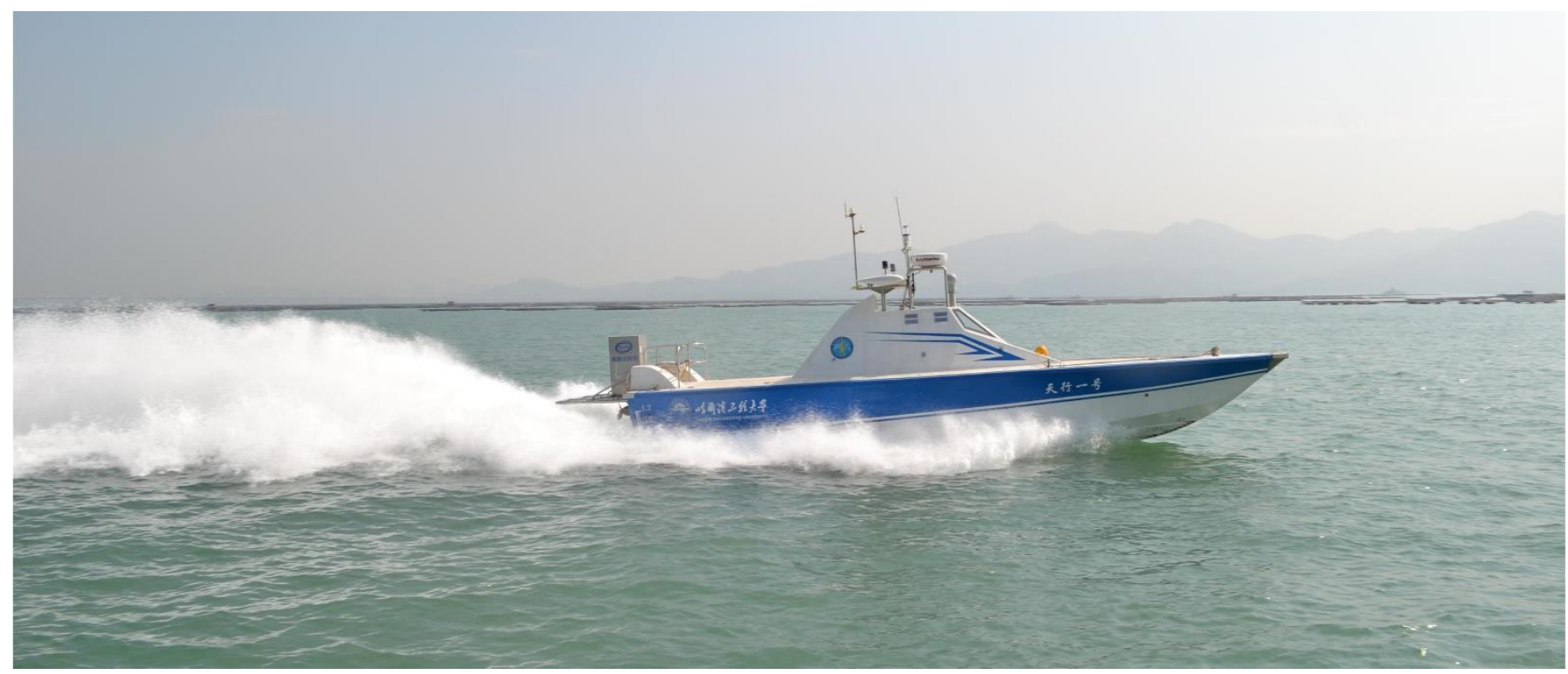

(a)

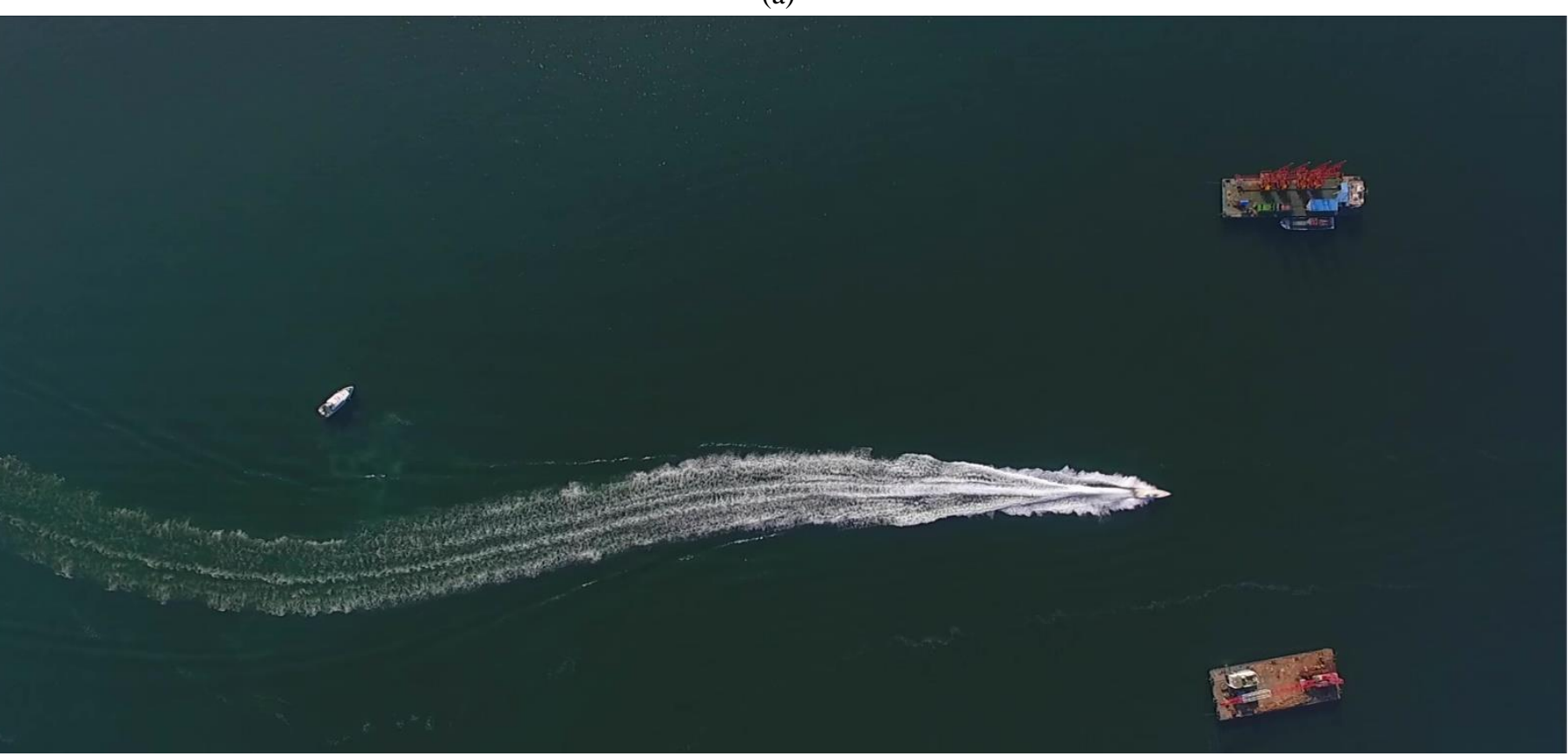

(b)

FIGURE 21 'Tianxing No.1' high-speed USV. (a) The high-speed sailing state of 'Tianxing No.1' USV. (b) Obstacle avoidance test

For the sea trials locations, two typical sea areas, Shenzhen Bay Port at Guangdong province, China and New Lvshun Port at Dalian, Liaoning province, China, are selected (shown in Figure 22). During the trials, practical static obstacles of moored ships are used to test the collision avoidance capability. To increase the complexity of the trials, ships are being selected randomly and in real-time, i.e. no a prior knowledge of the obstacle information exists making the algorithms being tested to the most extent. To monitor the trials and record experimental data, the position and attitude information of the USV (Figure 23) together with the 
environment perception video (Figure 24) is displayed on the monitoring system and transmitted back to a shore station via a radio communication channel. In Figure 24, four different environment perception information including visible light camera, infrared camera, radar and fused data is displayed and such a format will be used for presenting results in the following sections.

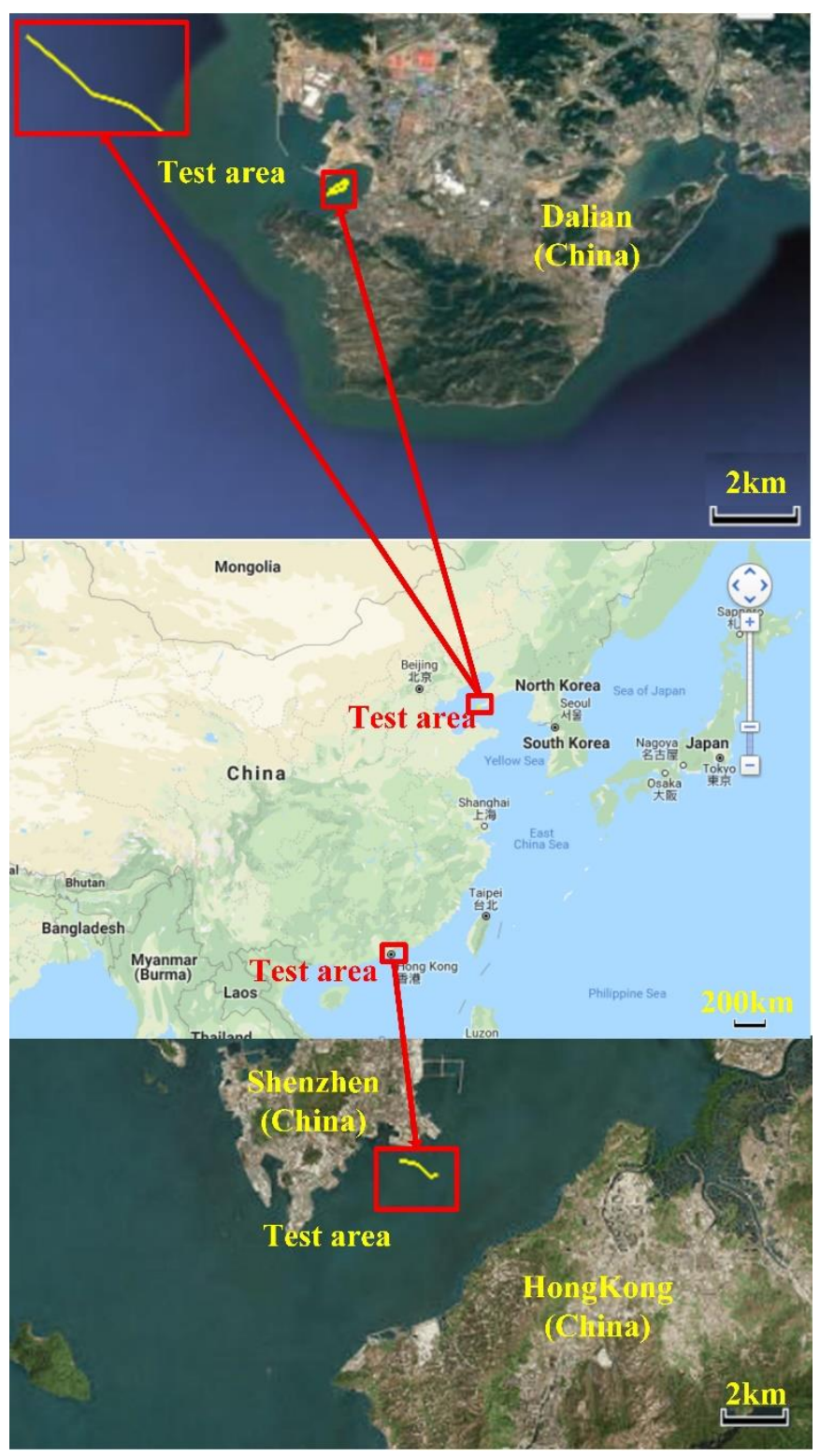

FIGURE 22 Test area 


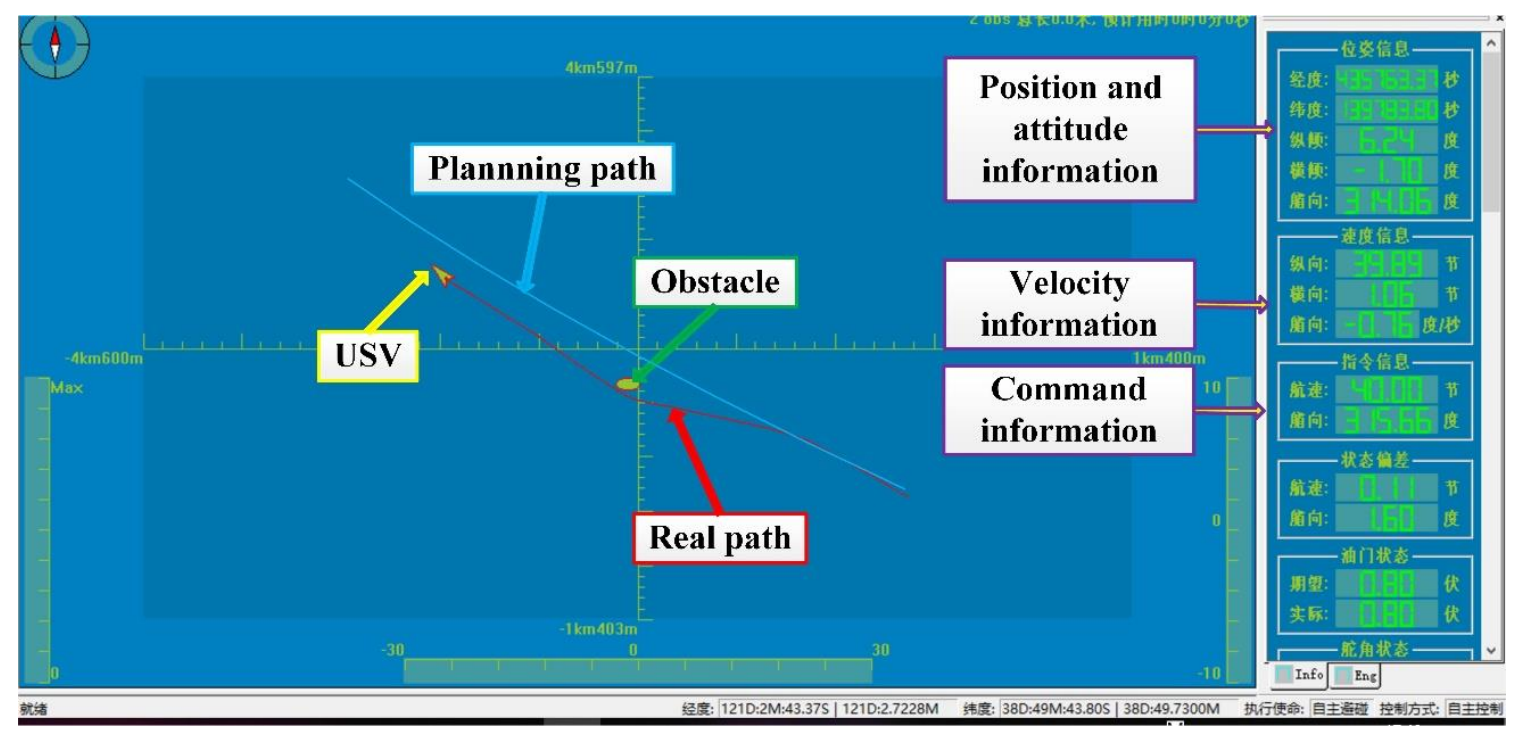

FIGURE 23 Monitoring system interface

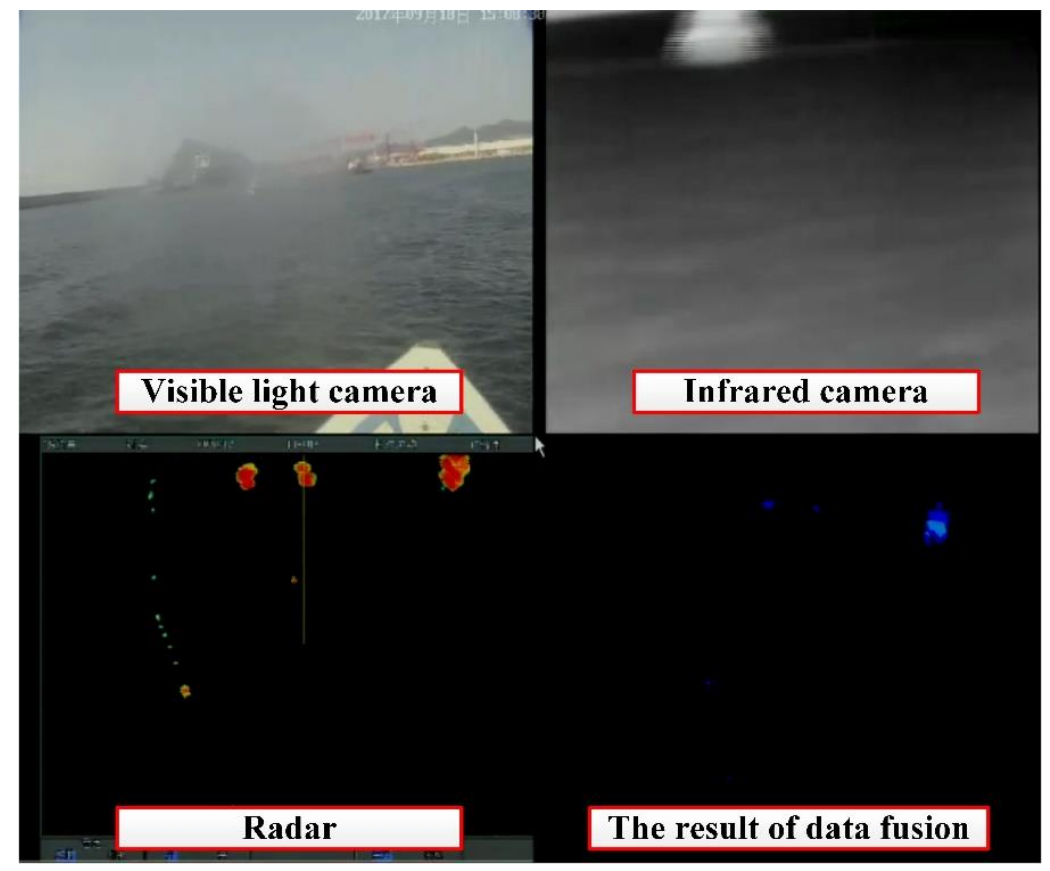

FIGURE 24 Environment perception system interface

\subsection{Multiple obstacles collision avoidance test}

\subsubsection{Collision avoidance test in constrained environment with sparse obstacles}

The initial test was carried out in the anchorage between Shenzhen and Hong Kong. As shown in Figure 25 , the dashed line is the planned route, the solid line is the USV actual tracking route and the red dots are obstacles. $S$ is the starting point, $G$ is the goal point. Due to the speed restrictions imposed by the local authority for safety reason, the USV's speed was limited to 5 knots. In Figure 25, the USV sailed from east to west to avoid two ships with the first ship being a fishing ship with the length of $10 \mathrm{~m}$ and the second ship being a 
mooring ship with length about $40 \mathrm{~m}$ length. The fishing ship was also being used as the tracking boat to record the process of collision avoidance. With the distance between two ships about $600 \mathrm{~m}$, a expended radius placed to obstacles to ensure navigation safety was configured to be $50 \mathrm{~m}$.

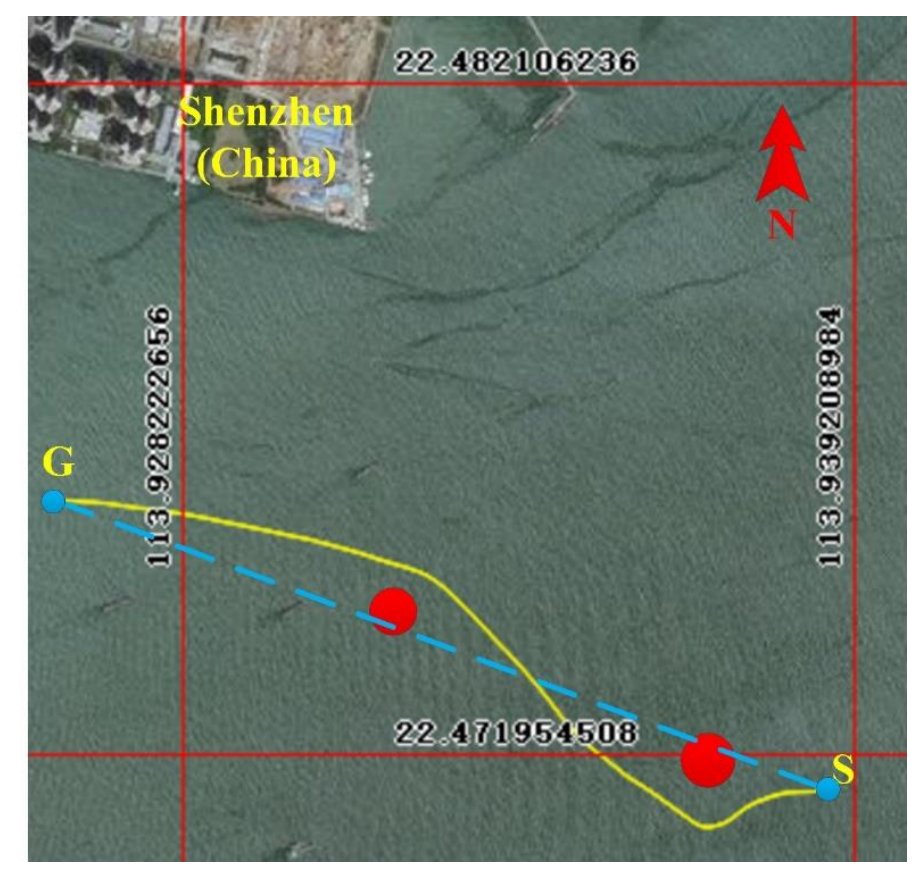

FIGURE 25 Multiple obstacles collision avoidance test at Shenzhen

The collision avoidance process, the expected and actual heading changes and the expected and actual speed of 'Tianxing No.1' are shown in Figure 26, Figure 27, Figure 28 and Figure 29, respectively. From Figure 28, it can be observed that two evasive actions were carried out from initial and at time step 356 (each time step is equivalent to $0.5 \mathrm{~s}$ ). Two issues of instability of obstacles and time-lag of high-speed USVs are evident in Figure 27 and Figure 28. In Figure 27, obstacle 2's size and position were different in different time step and in Figure 28 certain response time was required to adjust the USV's heading to the expected value.

For the collision avoidance performance (shown in Figure 28), during the initial stages, after the first obstacle been detect by the USV, a starboard manoeuvre was made by following the command of a gradual heading change from $273^{\circ}$ to $231^{\circ}$. At time step 180, avoidance with the first obstacle was completed and the heading was adjusted to make a left turning of $296^{\circ}$ towards the goal point. At time step 356, the USV detected the second obstacle and decided to change its heading to $302^{\circ}$ and made a port manoeuvre. The heading was increased from $302^{\circ}$ to $316^{\circ}$ for a proper collision avoidance and at time step 697 , the second evasive action was completed and the USV continued to navigate to the goal point. It should be noted that albeit the 
oscillation of the actual heading angle, a robustness in generating the control command is evident. A stable expected heading angle (red line in Figure 28) can always be provided due to the designed algorithms for obstacle stabilisation and heading maintenance. Also, as shown in Figure 29, the USV was sailing at a low speed with a stable speed distribution. A slight speed drop was caused by the continuous heading change but contained within a tolerant range.

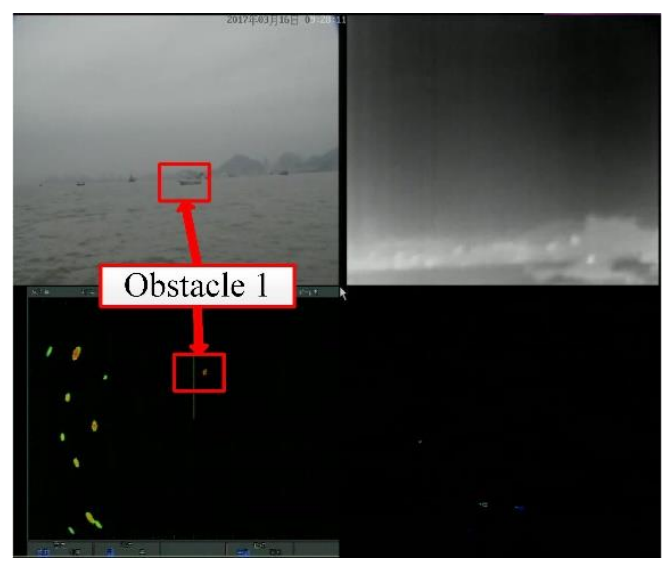

(a)

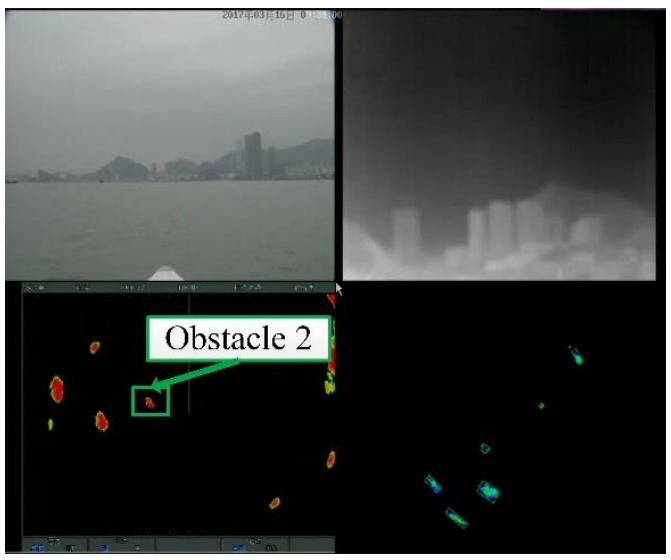

(c)

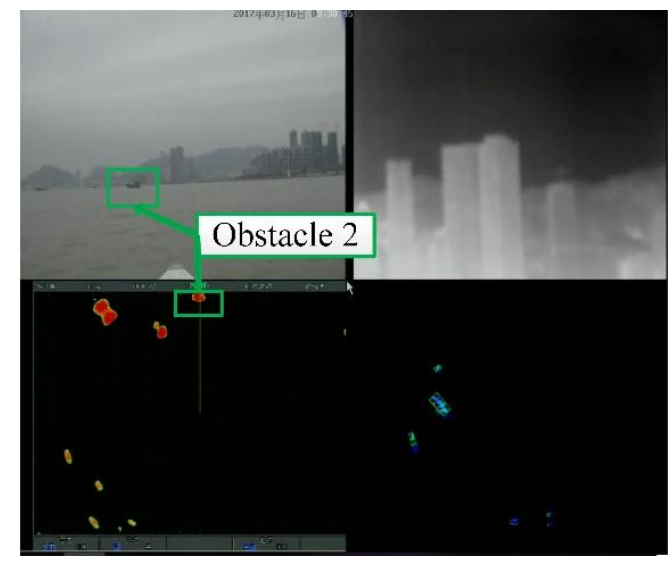

(b)

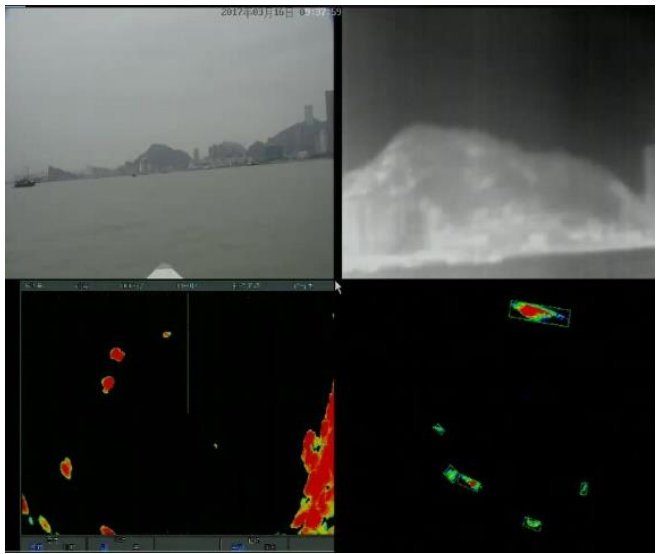

(d)

FIGURE 26 The process of collision avoidance test in environmental perception system. (a) Time step=0. (b) Time step=356.

(c) Time step $=697$. (d) Time step $=1000$

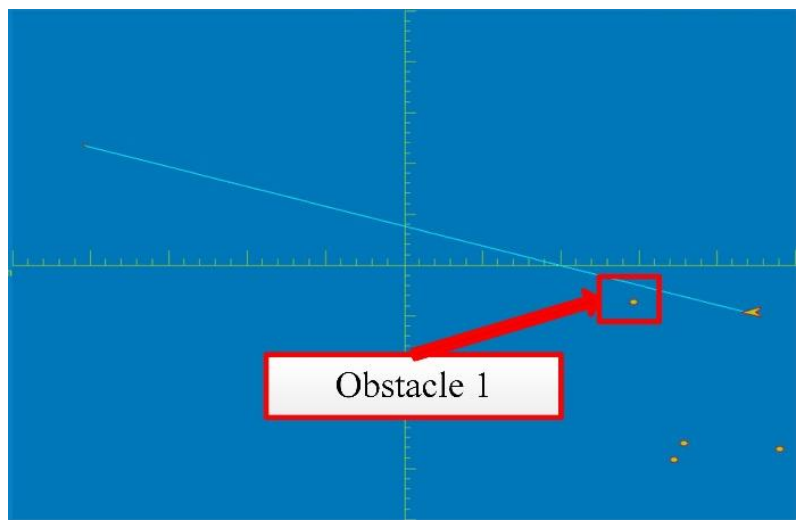

(a)

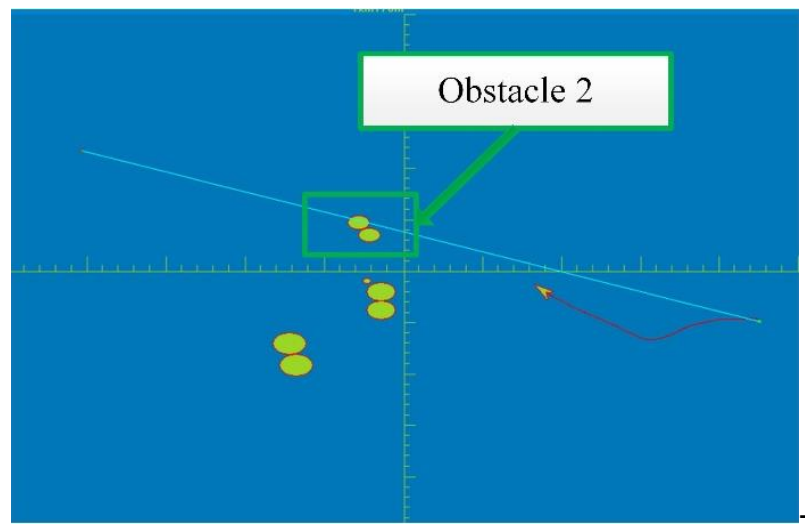

(b) 


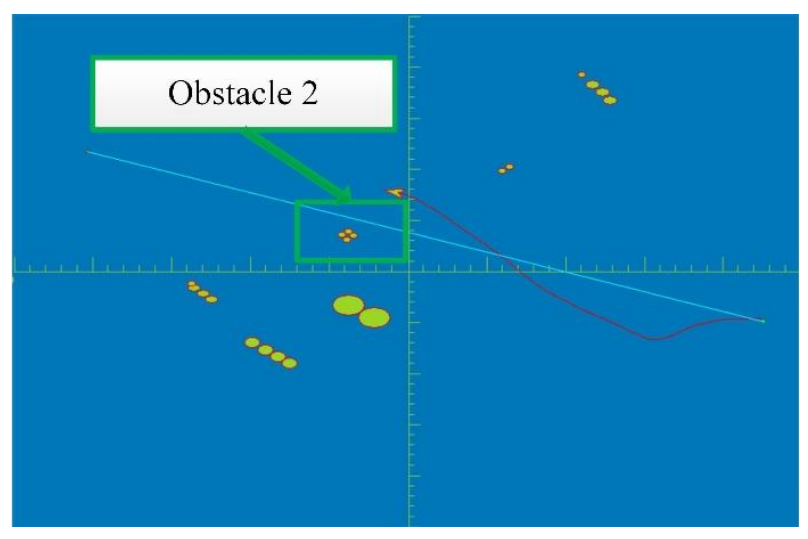

(c)

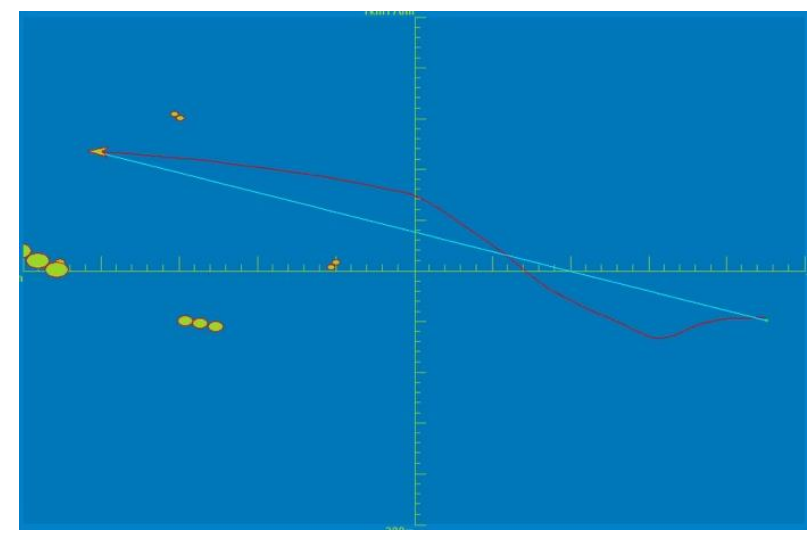

(d)

FIGURE 27 The process of collision avoidance test in monitoring system. (a) Time step=0. (b) Time step=356. (c) Time step=697. (d) Time $\mathrm{step}=1000$

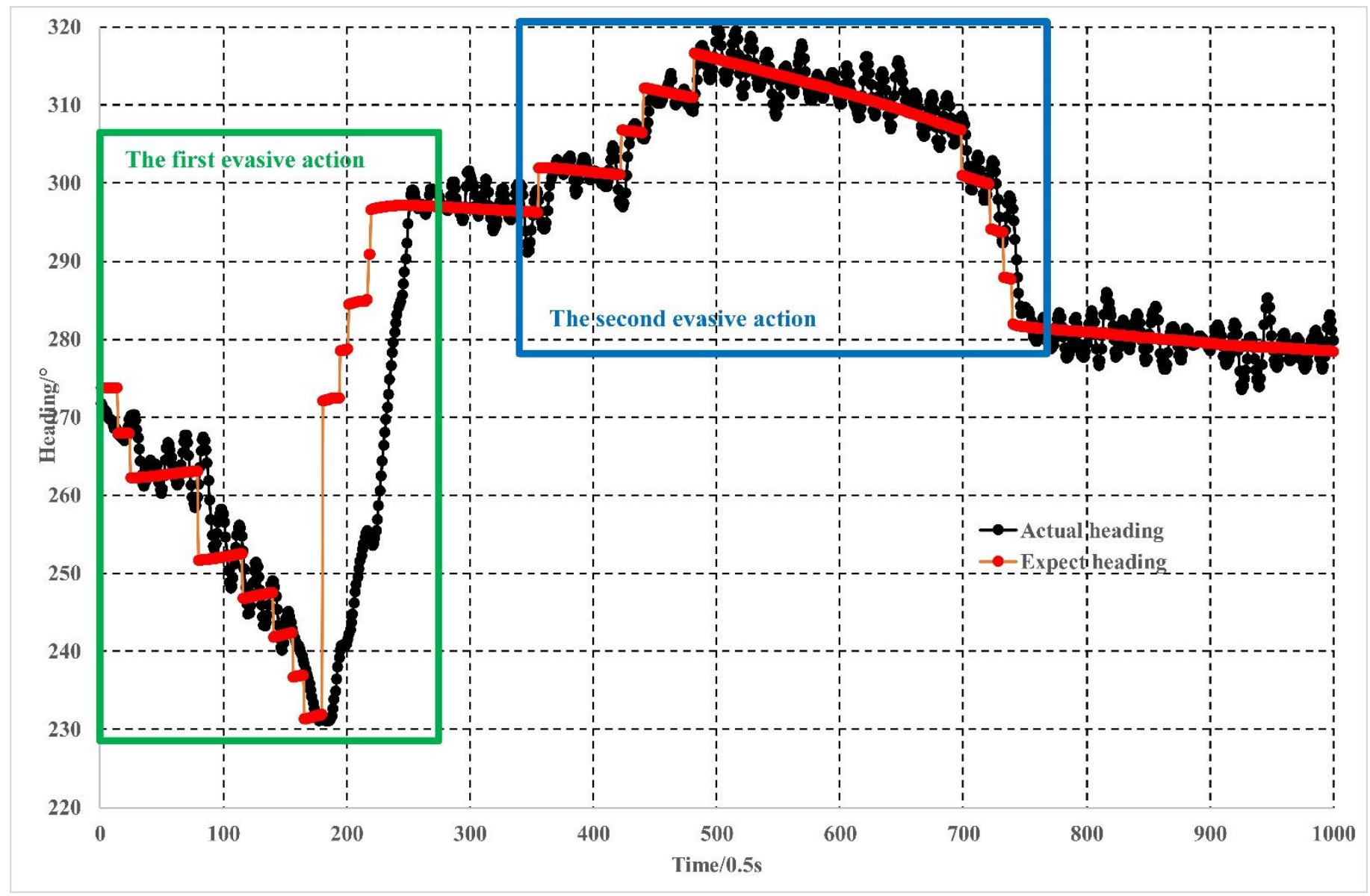

FIGURE 28 The heading of multi-obstacles collision avoidance test at Shenzhen 


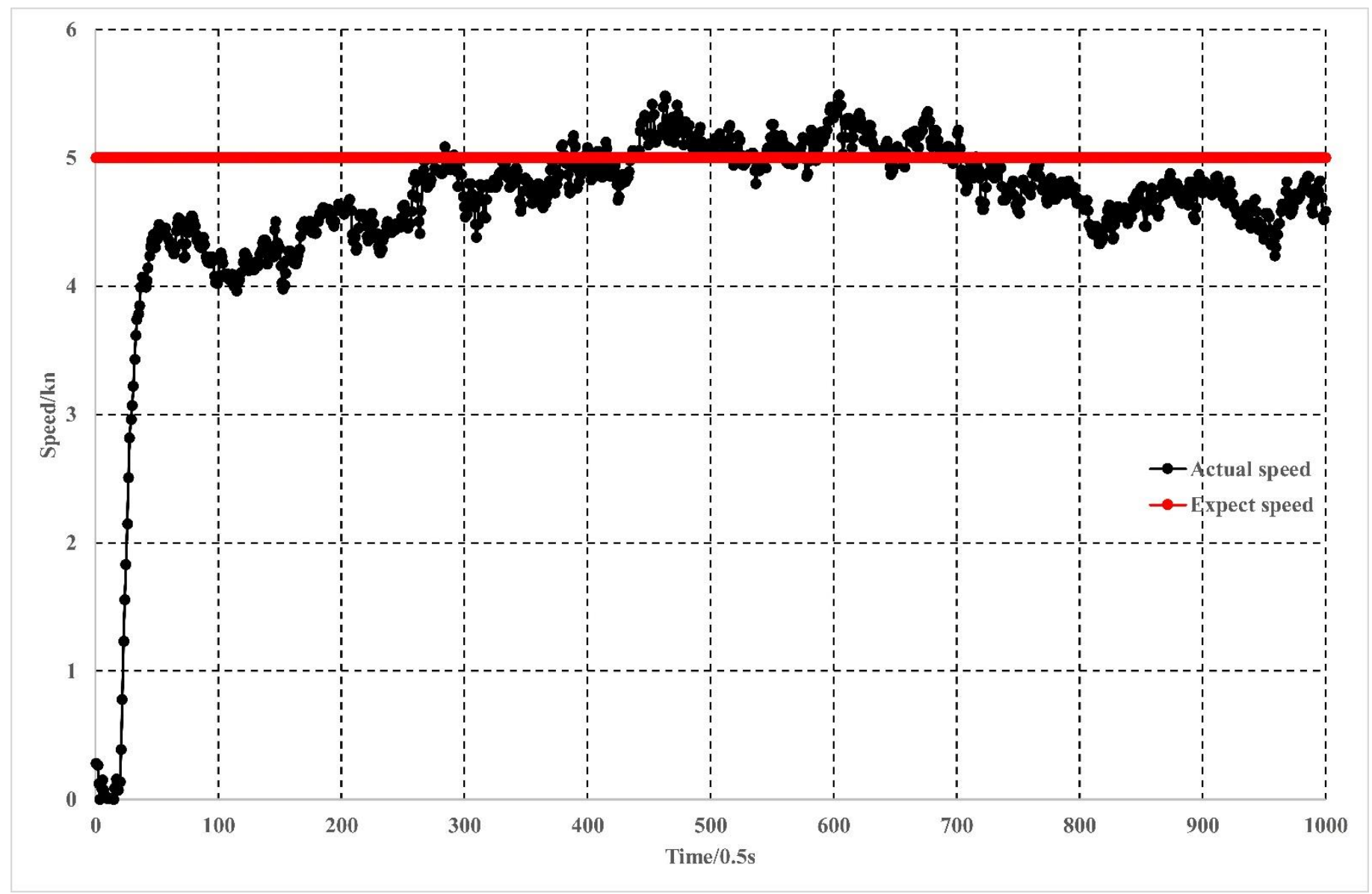

FIGURE 29 The speed of multi-obstacles collision avoidance test at Shenzhen

\subsubsection{Collision avoidance test in harbour areas with dense obstacles}

To further verify the obstacle avoidance capability, another set of trials were carried out in a harbour area at New Lvshun Port at Dalian, Liaoning province, China. The harbour can be regarded as a constrained environment with dense obstacles with various fishing ships travelling. The length of these fishing ships ranges from $5 \mathrm{~m}$ to $10 \mathrm{~m}$ and by adhering to the traffic regulations, the speed of 'Tianxing No.1' was limited to 8 knots.

As shown in Figure 30, the trial was designed to let the USV travel from southeast to northwest and return to the start point by avoiding 5 fishing ships en route. Actual distance between each pair of fishing ships was no more than $150 \mathrm{~m}$. Adding to that, due to the influences caused by sensor noises, the actual permissible travelling space between two obstacles was less than $100 \mathrm{~m}$ making the collision avoidance complicated. 


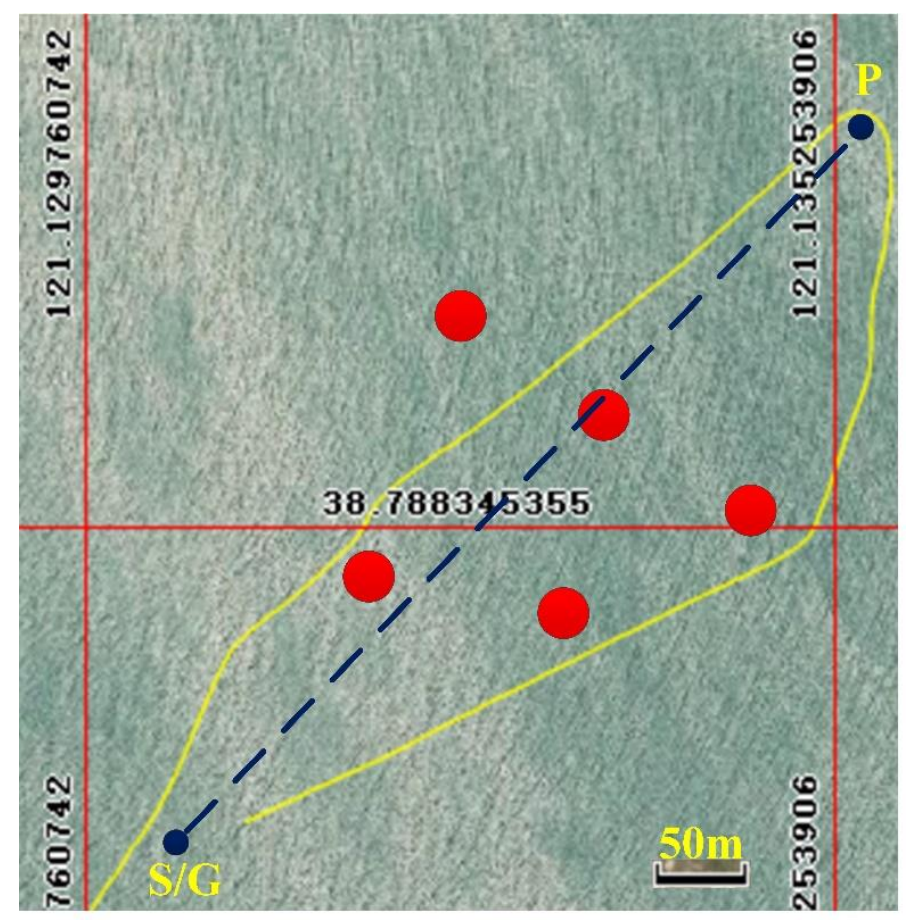

FIGURE 30 Multiple obstacles collision avoidance test

The trace of 'Tianxing No.1' is shown as the yellow lines in Figure 30, it demonstrates that the USV can successfully avoid obstacles (red circles) while navigating. Detailed collision avoidance process is shown in Figure 31 with the variations of heading and speed of the USV represented in Figure 32 and Figure 33. As shown in Figure 31, four evasive actions were taken by 'Tianxing No.1'. A stable control command (the expected heading angle shown as the red line in Figure 32) can always be generated to ensure that a consistent evasive action was implemented. Note that such a strategy is critical for high-speed USVs encountering with cluttered obstacles as any inconsistency in manoeuvre would lead to unexpected collisions. The consistency in control command can also be observed in speed control as shown in Figure 33. The speed of 'Tianxing No.1' can well stay around the expected speed ( 8 knots) with the exception at time step 382 . This phenomenon was mainly caused by the repeating change of the USV's heading but the speed drop was retained to be less than 2 knots, which had limited influence on navigation. 


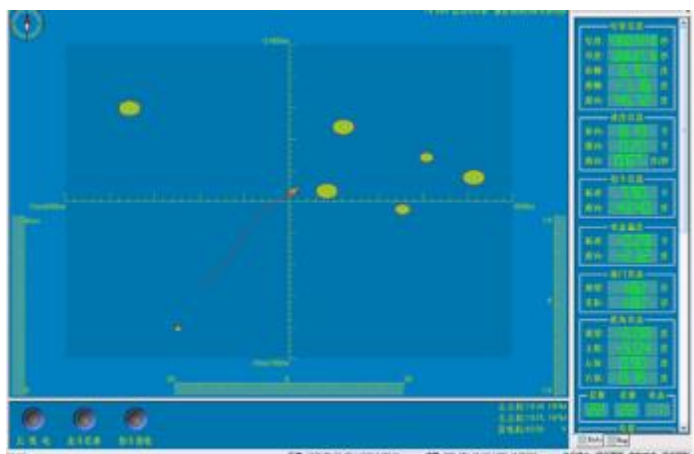

(a)

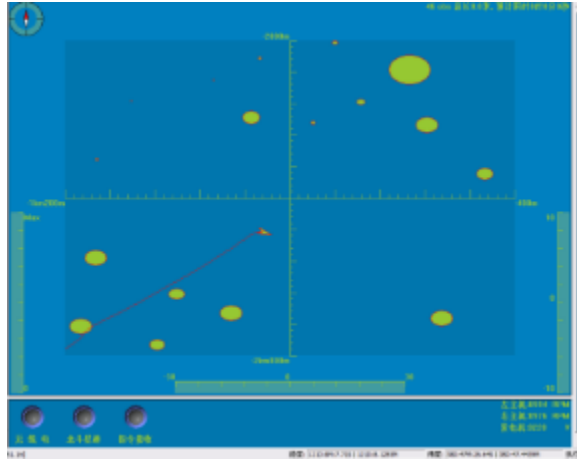

(c)

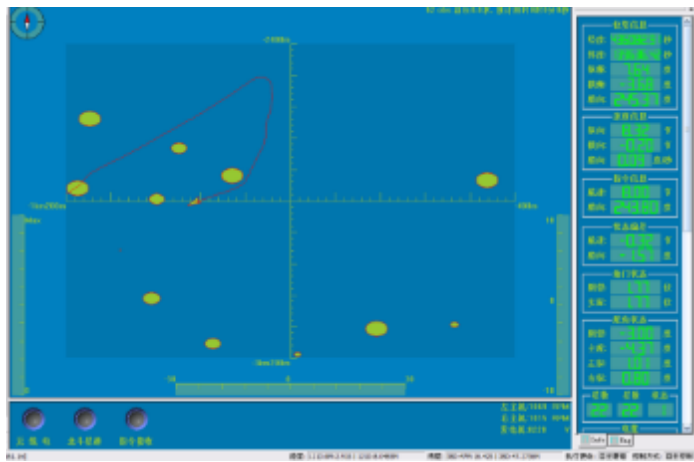

(e)

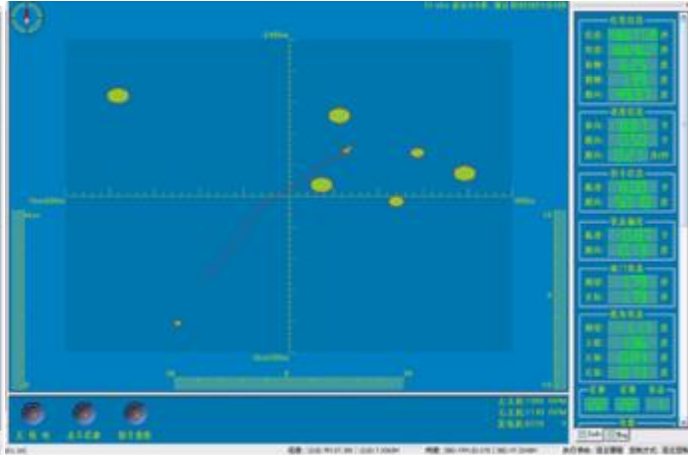

(b)

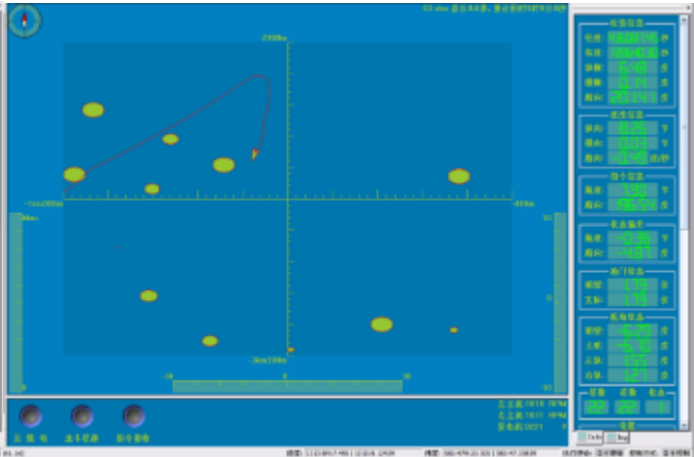

(d)

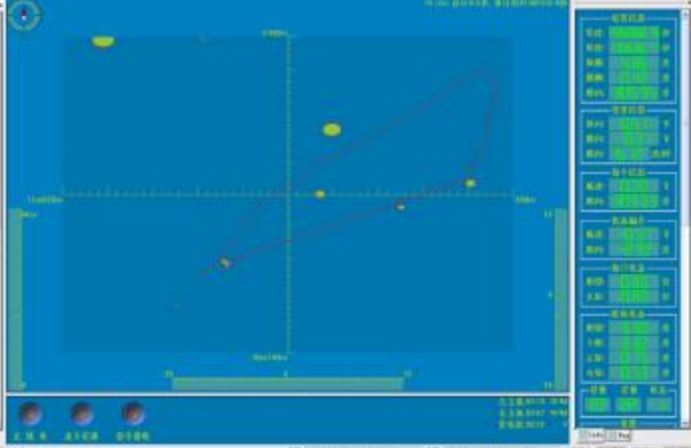

(f)

FIGURE 31 The process of multi-obstacles collision avoidance test. (a) Time step=92. (b) Time step=143. (c) Time step=303. (d) Time $s$ tep $=422$. (e) Time $s t e p=500$. (f) Time step $=666$ 


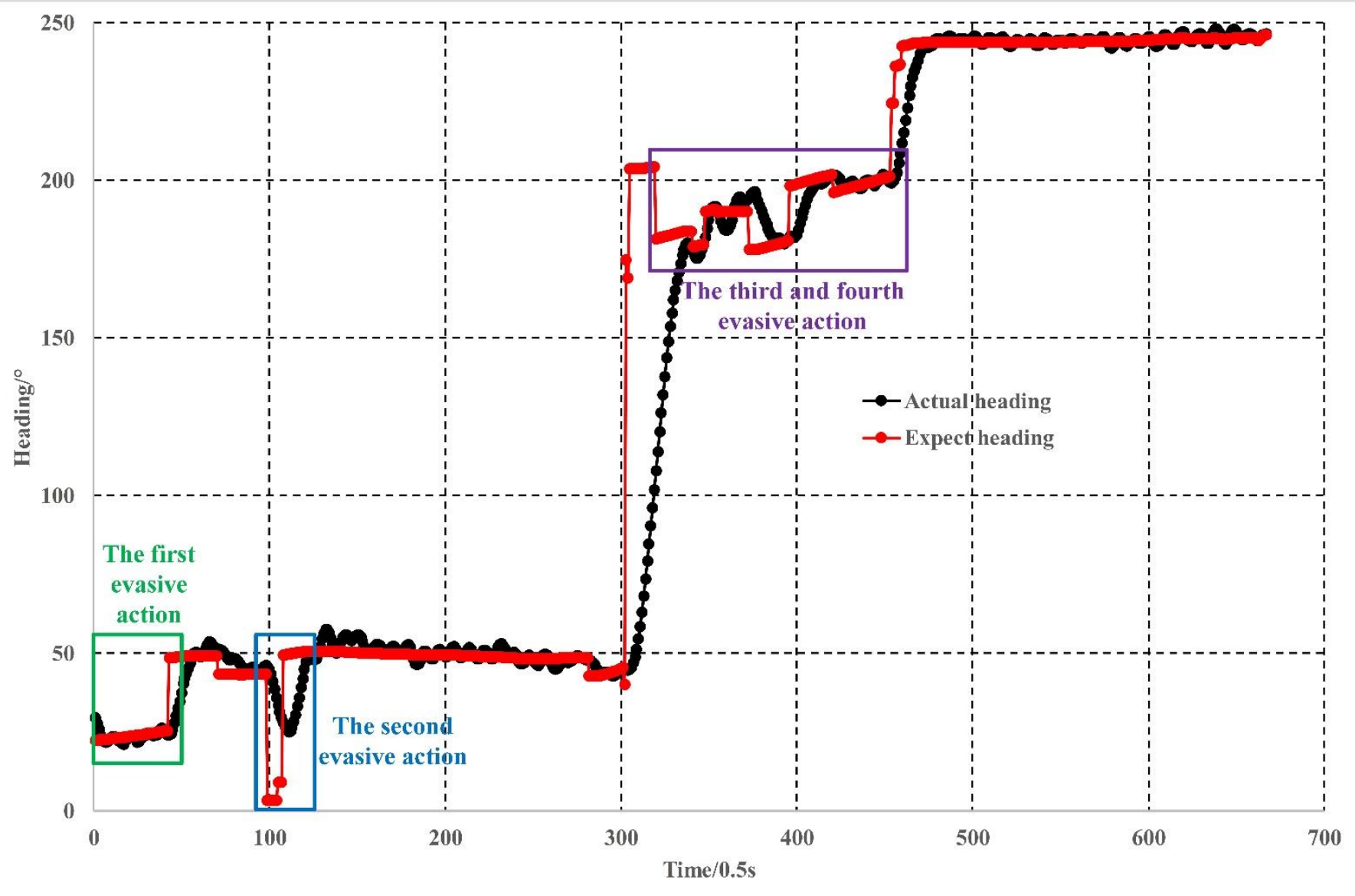

FIGURE 32 The heading of multi-obstacles collision avoidance test

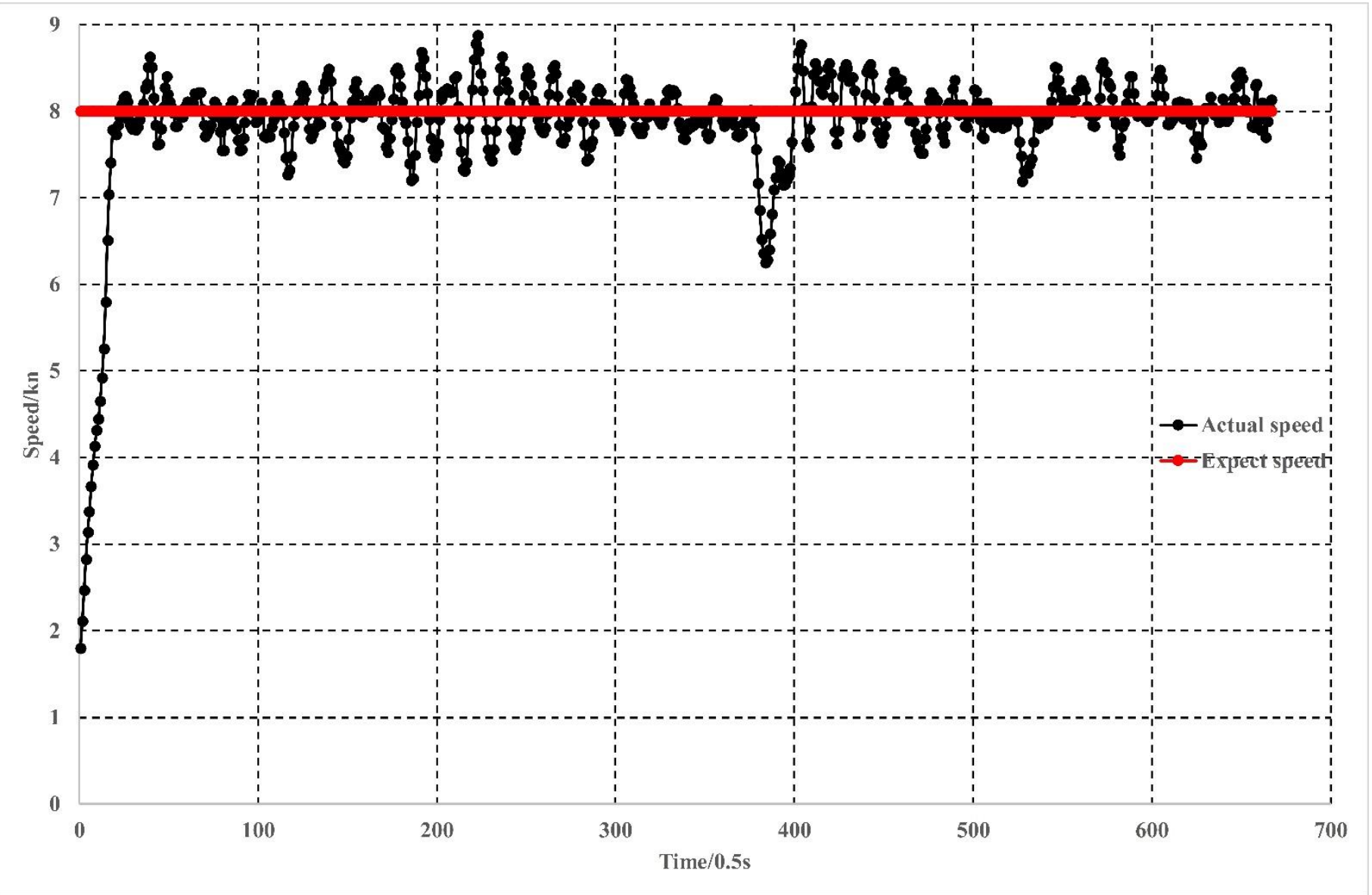

FIGURE 33 The speed of multi-obstacles collision avoidance test 


\subsection{Collision avoidance test in open sea areas with high speed}

The final test was designed to demonstrate the collision avoidance under high-speed in open sea areas. 'Tianxing No.1' has the maximum speed of 50 knots with the cruising speed of 40 knots. Therefore, in this trial, the USV was commanded to travel at the speed of 40 knots from southeast to northwest to avoid a moored ship. The length of moored ship is about $100 \mathrm{~m}$ and due to the influence of the anchor chain, the moored ship was given an expended radius of $100 \mathrm{~m}$. Figure 34 shows the trajectory (yellow line) of 'Tianxing No.1', which proves that the moored ship can be well avoided and the USV reached the target successfully.

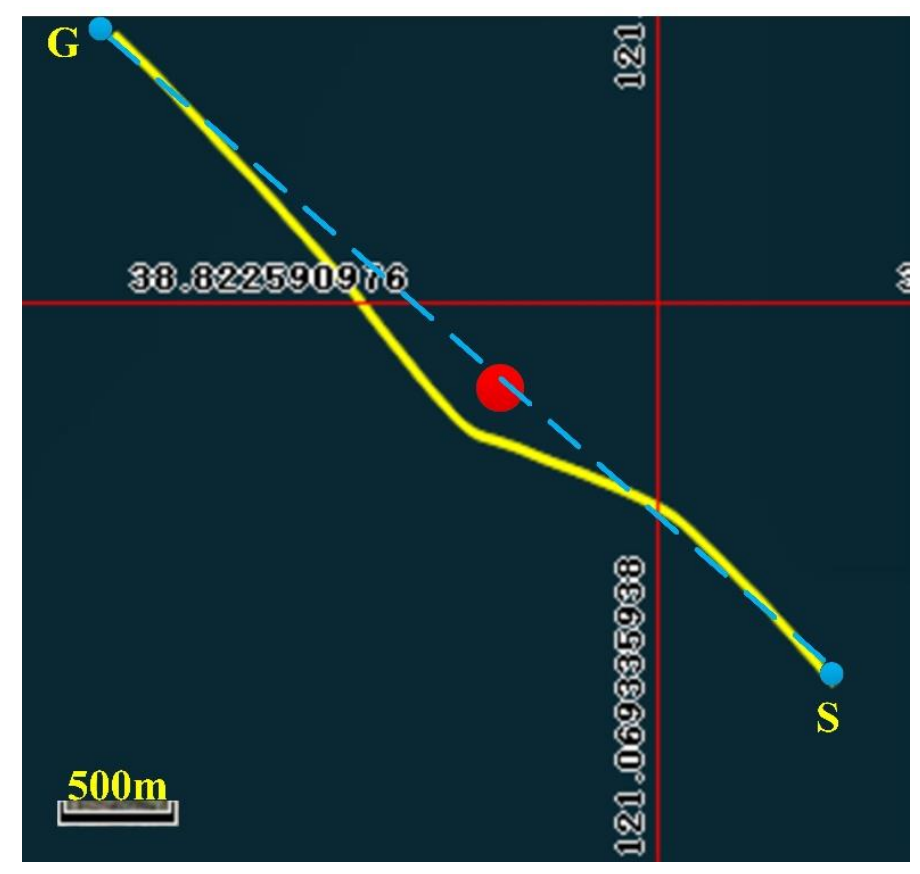

FIGURE 34 High-speed obstacle collision avoidance test

The detailed avoidance process is shown in Figure 35 with the variations of heading and speed shown in Figure 36 and Figure 37. In Figure 36, in initial stages, the expect heading $\alpha_{\text {expect }}$ was about $310^{\circ}$ and the USV was travelling to the goal point at 40 knots speed. At time step 129, the USV starts avoiding the obstacle with the expected heading $\alpha_{\text {expect }}$ adjusted to $305^{\circ}$. During the process of obstacle avoidance, seven more adjustments were made in real-time to change the expected heading from $305^{\circ}$ to $276^{\circ}$. After time step 276 , the USV successfully passed the obstacle and $\alpha_{\text {expect }}$ was changed back to $\alpha_{\text {goal }}$. As shown in Figure 37 , the speed of USV can again be retained close to 40 knots, which demonstrates the effectiveness of the proposed algorithm. 


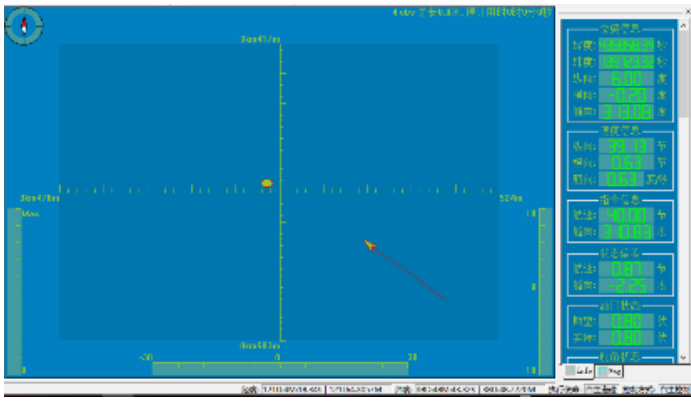

(a)

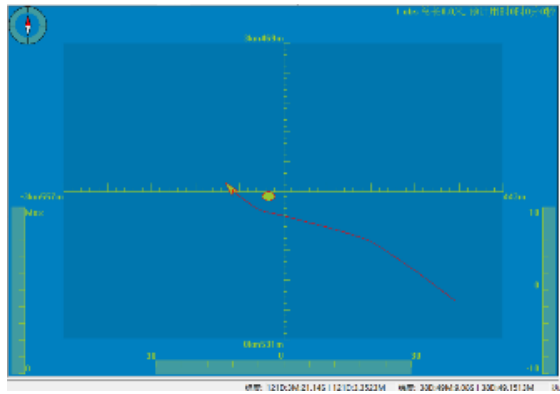

(c)

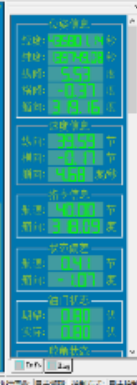

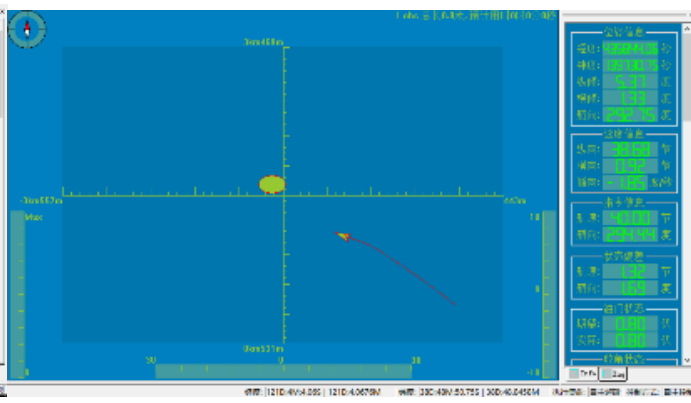

(b)

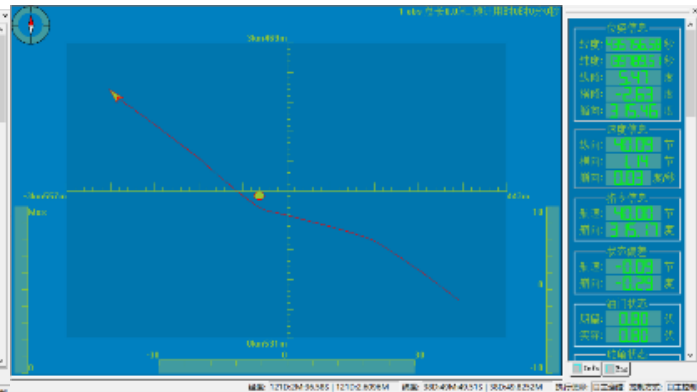

(d)

FIGURE 35 The process of high-speed collision avoidance test. (a) Time step=70. (b) Time step=180. (c) Time step $=300$. (d) Time step $=550$.

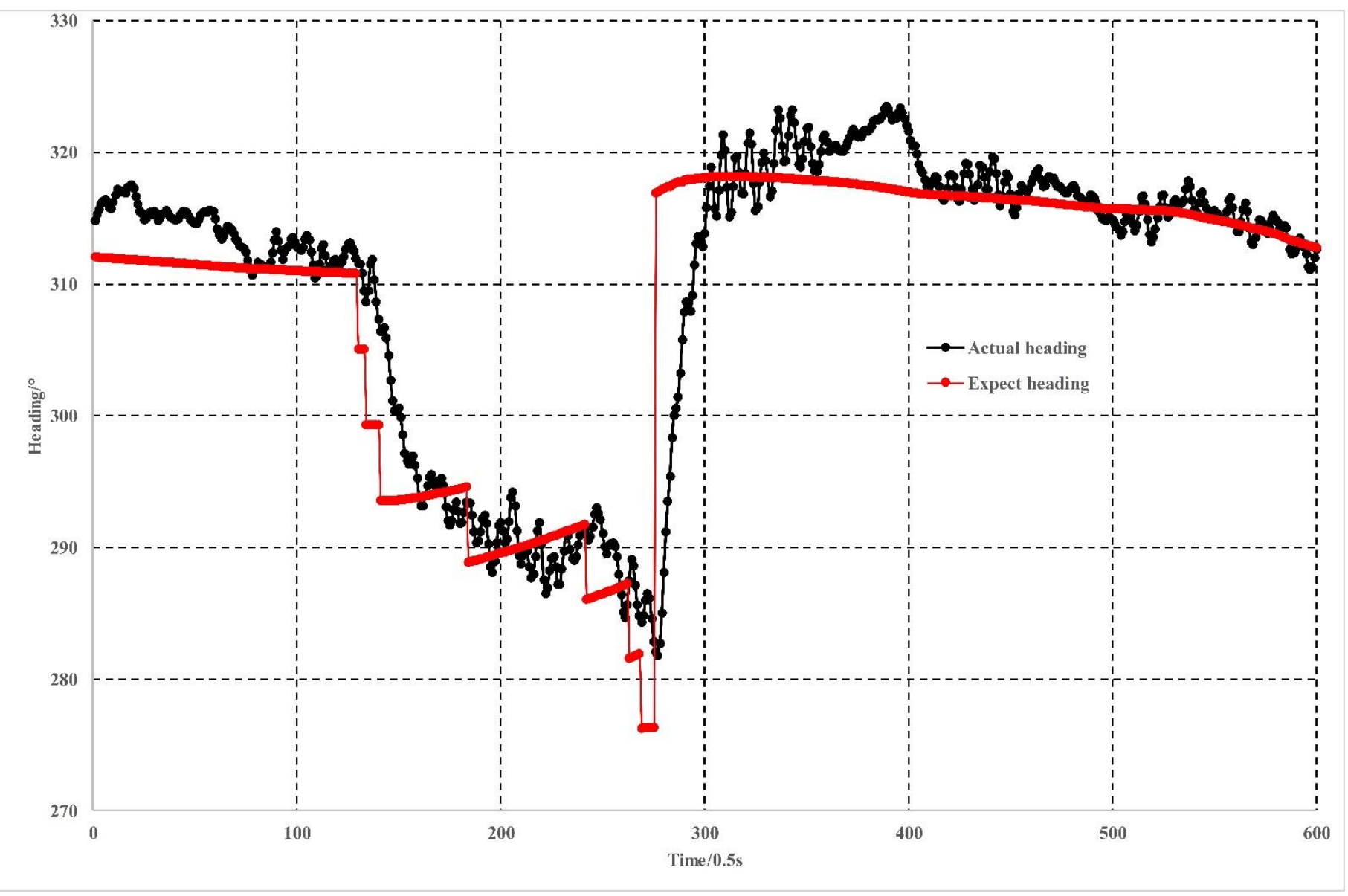

FIGURE 36 The heading of high-speed collision avoidance test 


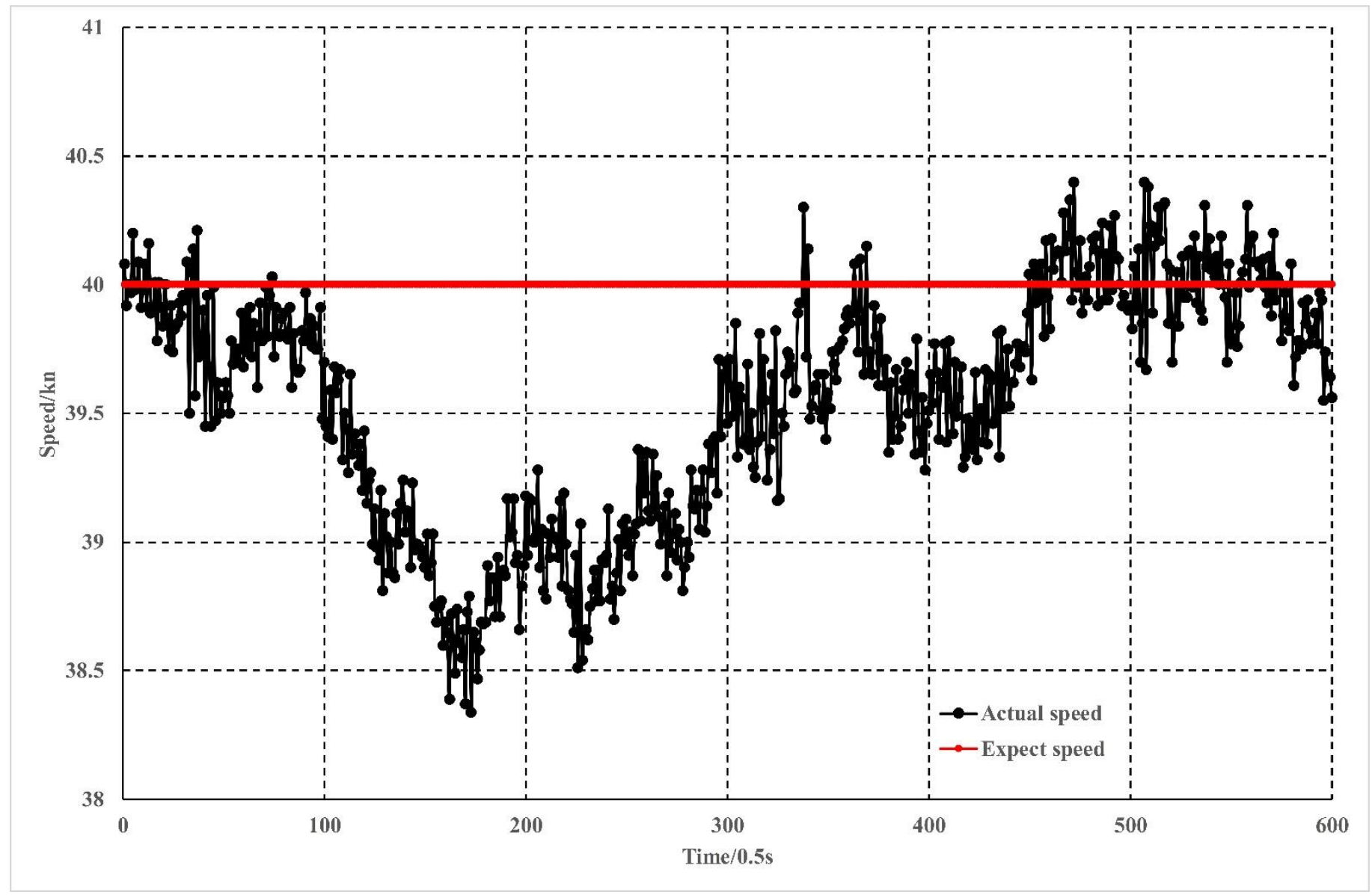

FIGURE 37 The speed of high-speed collision avoidance test

\section{Conclusions and learned lessons}

In this paper, an autonomous navigation system specifically designed for high-speed USVs is proposed with system's stability, reliability, and real-time capability been verified in various simulation tests and sea trials. To resolve the problem of obstacles' instability and improve the control robustness, a local environment model generation method and a heading maintaining method have been designed and integrated into the collision avoidance approach. By installing the designed autonomous navigation system onto a high-speed USV platform of 'Tianxing No.1', experiment results show that the autonomous navigation system can guide the high-speed USV navigate in various maritime environments with guaranteed safety.

From the conducted sea trials, lessons in regard to improve the capability of USVs in general can be learned. In particular, based upon our experiences in designing, building and testing our 'Tianxing No.1' highspeed USV, crucial aspects in a holistic USV operation process, especially for high-speed USV, can be learned. Important knowledge obtained for the design and integration of hardware and software can be used in future 
work to further improve the autonomy level of USVs. Some critical learned lessons are listed as follows:

1) The system cooling problem: Each onboard equipment has its own rated working temperature, i.e. the maximum multi-board embedded industrial computer working temperature is $70^{\circ} \mathrm{C}$ and the IMU based on fiber optic gyroscope (FOG) has the maximum working temperature of $60^{\circ} \mathrm{C}$. Any working temperature exceeding the rated value would lead to issues of inaccurate sensor data and irreversible damage to devices. Apart from the high ambient temperature that may influence the sensors' working temperature, devices themselves will also generate heat which contributes to the increase of temperature. For example, the powerful vision computer deals with vast vision data and process them in real-time, which potentially generate heat that should be dissipated timely. In our sea trials, an active air ventilation system was adopted in both hulls and control boxes. Also, the vision computer was installed in a separate box to mitigate the heat interference to other equipment.

2) The electromagnetic compatibility (EMC) problem: Three communication modes including network, radio and satellite communication were used for 'Tianxing No.1' high-speed USV. In order to enhance the communication robustness when the USV is far away from the shore station, a $20 \mathrm{~W}$ radio was selected to improve the communication range. However, the radio antenna will have a great influence on GPS signal, especially in rainy or foggy weather making GPS speed and position data error outside the tolerance of the system in some cases. On 'Tianxing No.1', the radio antenna and GPS antenna are allocated at separate places to resolve such an issue. Also, when the GPS signal is affected, an alternative, the Beidou signal, will be considered.

3) The hydraulic actuator's time-lag problem: The steering control of water-jet and surface-drive propulsion system is achieved by hydraulic actuators, time-lag of which is much larger than that of motor actuator. The total time from hard port to hard starboard for 'Tianxing No.1' is about $10 \mathrm{~s}$ making a more robust adaptive motion control strategies need to be developed for high-speed USV.

4) The components and sensors shock resistance problem: When the high-speed USV travelling at planning mode, a severe hull slamming will happen due to the wave affect. The shock resistance of components and sensors used for high-speed USV therefore needs to be fully considered. It is necessary to adopt a shock absorption measures for precision sensor, such as IMU. 


\section{Acknowledgements}

This work was financially supported by the National Natural Science Foundation of China (Grant NO. 52071100 and 51409054), National High Technology Research and Development Program of China (2014AA09A509). China Scholarship Council State Scholarship Fund (No. 201806685029)

\section{References}

The navy Unmanned Surface Vehicle (USV) master plan. (2007). https://www.navy.mil/navydata/technology/usvmppr.pdf

Kitts, C., Mahacek, P., Adamek, T., Rasal, K., Howard, V., \& Li, S. (2012). Field operation of a robotic small waterplane area twin hull boat for shallow-water bathymetric characterization. Journal of Field Robotics, 29(6), 924-938.

Bertaska, I. R., Shah, B., Von Ellenrieder, K., Svec, P., Klinger, W., \& Sinisterra, A. J. (2015). Experimental evaluation of automatically-generated behaviors for usv operations. Ocean Engineering, 106, 496-514.

Manley, J. E., (2008). Autonomous surface vessels, 15 years of development, Proc Oceans 2008 MTS/IEEE Quebec Conference and Exhibition (pp. 1-4), Quebec, Canada.

Sharma, S. K., Sutton, R., Motwani, A., \& Annamalai, A. (2013). Non-linear control algorithms for an unmanned surface vehicle. The International Journal of Maritime Engineering, 228(2), 146-155.

Caccia, M., Bibuli, M., \& Bono, R. (2008). Basic navigation, guidance and control of an unmanned surface vehicle. Autonomous Robots, 25 (4), 349-365.

Xu, T., Chudley, J., \& Sutton, R. (2006). Soft computing design of a multisensory data fusion system for unmanned surface vehicle navigation, Proceedings of the 7th IFAC Conference on Maneuvering and Control of Marine Craft (pp. 124-156). Lisbon, Portugal.

Yan, R. J., Pang, S., \& Sun, H.B. (2010). Development and missions of unmanned surface vehicle, Journal of Marine Science and Application, 9 (4), 451-457.

Sutton, R., Sharma, S., \& Xao, T. (2011). Adaptive navigation (pp. 124-156). systems for an unmanned surface vehicle, Journal of marine engineering and technology, 10 (3), 3-20.

Pieterkosky, S., Ziegwied, A., Cavanagh, C., \& Thompson, L. (2017). BIV meets ASV: Bio-inspired fish drones and autonomous surface vehicles for coral reef monitoring, OCEANS 2017 - Anchorage (pp. 15). Anchorage, AK, United States.

Iovino, S., Savvaris, A., Tsourdos, A. (2018). Experimental Testing of a Path Manager for Unmanned Surface Vehicles in Survey Missions, IFAC-PapersOnLine, 51 (29), 226-231.

Alves, J., Oliveira, R., Pascoal, A., Rufino, M., Sebastiao, L., \& Silvestre, C. (2006). Vehicle and Mission Control of the DELFIM Autonomous Surface Craft, 14th Mediterranean Conference on Control and Automation (pp. 1-6). Ancona, Italy. 
Han, J., Cho, Y., Kim, J., Kim, J., \& Kim, S. Y. (2020). Autonomous collision detection and avoidance for ARAGON USV: Development and field tests. Journal of Field Robotics.2020, 1-16.

Lee, T., Kim, H., Chung, H., Bang, Y., \& Myung, H. (2015). Energy efficient path planning for a marine surface vehicle considering heading angle. Ocean Engineering, 107, 118-131.

Singh, Y., Bibuli, M., Zereik, E., Sharma, S., Khan, A., \& Sutton, R. (2020). A novel double layered hybrid multi-robot framework for guidance and navigation of unmanned surface vehicles in a practical maritime environment. Journal of Marine science and Engineering, 8(9), 624.

Mina, T., Singh, Y., \& Min B. C., (2020). Maneuvering Ability-Based Weighted Potential Field Framework for Multi-USV Navigation, Guidance, and Control. Marine Technology Society Journal, 54(4), 40-58.

Wang, H., Wei, Z., Wang, S., Ow, C. S., Ho, K. T., \& Feng, B. (2011). A vision-based obstacle detection system for Unmanned Surface Vehicle. 2011 IEEE 5th International Conference on Robotics, Automation and Mechatronics (pp. 364-369). Qingdao, China.

Hermann, D., Galeazzi, R., Andersen, J. C., \& Blanke, M. (2015). Smart Sensor Based Obstacle Detection for High-Speed Unmanned Surface Vehicle. 10th IFAC Conference on Manoeuvring and Control of Marine Craft (pp. 90-197). Copenhagen, Denmark.

Almeida, C., Franco, T., Ferreira, H., Martins, A., Santos, R., Almeida, J. M., Carvalho, J., \& Silva, E. (2009). Radar based collision detection developments on USV ROAZ II, OCEANS 2009-EUROPE (pp. 1-6), Bremen, Germany.

Martins, A., Almeida, J. M., Ferreira, H., Silva, H., Dias, N., Dias, A., Almeida, C., \& Silva, E. P. (2007). Autonomous Surface Vehicle Docking Manoeuvre with Visual Information, Proceedings 2007 IEEE International Conference on Robotics and Automation (pp. 4994-5000), Rome, Italy.

Oleynikova, E., Lee, N. B., Barry. A. J., Holler, J., \& Barrett, D. (2010). Perimeter patrol on autonomous surface vehicles using marine radar. OCEANS 2010 (pp. 1-5). Sydney, Australia.

Bohren, J., Foote, T., Keller, J., Kushleyev, A., Lee, D., Stewart, A., Vernaza, P., Derenick, J., Spletzer, J., \& Satterfield, B. (2008). Little Ben: The Ben Franklin racing team's entry in the 2007 DARPA urban challenge, Journal of Field Robotics, 9(25),598-614.

Urmson, C., Anhalt , J., \& Bagnell, D. (2008). Autonomous driving in urban environments: Boss and the urban challenge. Journal of Robotic Systems, 8(25), 425-466.

Halterman, R., \& Brush M. (2010). Velodyne HDL-64E LIDAR for Unmanned Surface Vehicle Obstacle Detection, Proceedings of SPIE - The International Society for Optical Engineering (pp. 7692, 9), Orlando, United States.

Zhuang, J. Y., Zhang, L., Zhao, S. Q., Cao, J., Wang, Bo., \& Sun, H. B. (2016). Radar-based collision avoidance for unmanned surface vehicle. China Ocean Engineering, 30 (6), 867-883.

Park, J., Kang, M., Kim, T., Kwon, S., Han, J., \& Wang, J., et al. (2017). Development of an Unmanned Surface Vehicle System for the 2014 Maritime RobotX Challenge. Journal of field robotics, 34 (4), 644 - 
665.

Holler, J., Longfield, S., Murphy, K., Striz, A., \& Bingham, B. (2008). Unmanned surface vehicle for undergraduate engineering science education, Oceans 2008 (pp. 1-8). Quebec, Canada.

Casalino, G., Turett,a A., \& Simetti, E. (2009). A Three-Layered Architecture for Real Time Path Planning and Obstacle Avoidance for Surveillance USVs Operating in Harbour Fields, OCEANS 2009-EUROPE (pp. 1-8). Bremen, Germany.

Larson, J., Bruch, M., \& Ebken, J. (2006). Autonomous navigation and obstacle avoidance for unmanned surface vehicles. Proceedings of SPIE - The International Society for Optical Engineering (pp. 623007: 1-12). Orlando, United States.

Huntsberger, T., Aghazarian, H., Howard, A., \& Trotz, D. C. (2011). Stereo Vision-Based Navigation for Autonomous Surface Vessels. Journal of Field Robotics, 28(1), 3-18.

Lolla, T., Lermusiaux, P., Ueckermann, M., \& Haley, P., (2014). Time-optimal path planning in dynamic flows using level set equations: theory and schemes. Theoretical, Computational and Observational Oceanography, 64 (10), 1373-1397.

Liu, Y., \& Bucknall, R. (2015). Path planning algorithm for unmanned surface vehicle formations in a practical maritime environment. Ocean Engineering, 97, 126-144

Song, R., Liu, Y., \& Bucknall, R. (2017). A multi-layered fast marching method for unmanned surface vehicle path planning in a time-variant maritime environment. Ocean Engineering, 129, 301-317

Naeem, W., Irwin, G. W., \& Yang, A. (2012). COLREGs-based collision avoidance strategies for unmanned surface vehicles. Mechatronics, 22(6), 669-678.

Tam, C., \& Bucknall. R. (2010). Path-planning algorithm for ships in close-range encounters. Journal of Marine Science and Technology, 15 (4), 395-407.

Tsou, M. C., \& Hsueh, C. K. (2010). The study of ship collision avoidance route planning by ant colony algorithm, Journal of Marine Science and Technology. 18(5), 746-756.

Thakur, A., Svec, P., \& Gupta, S. K. (2012). GPU based generation of state transition models using simulations for unmanned surface vehicle trajectory planning. Robotics and Autonomous Systems, 60(12), 1457-1471.

Ma, Y., Hu, M., \& Yan X. (2018). Multi-objective path planning for unmanned surface vehicle with currents effects. ISA Trans., 75, 137-156

Niu, H., Ji, Z., Savvaris, A., \& Tsourdos A. (2020). Energy efficient path planning for Unmanned Surface Vehicle in spatially-temporally variant environment, Ocean Engineering, 196, 106766

Sombolestan1, S. M., Rasooli A., \& Khodaygan S. (2019). Optimal path-planning for mobile robots to find a hidden target in an unknown environment based on machine learning. Journal of Ambient Intelligence and Humanized Computing, 10, 1841-1850

Saha, O. \& Dasgupta, P. (2017). Real-Time Robot Path Planning Around Complex Obstacle Patterns through Learning and Transferring Options. IEEE International Conference on Autonomous Robot Systems and 
Competitions (ICARSC) (pp. 278-283). Coimbra, Portugal.

Choi, S.Y., \& Cha, D. (2019). Unmanned aerial vehicles using machine learning for autonomous flight; stateof-the-art, Advanced Robotics, 33(6), 265-277.

Vibhute, S. (2018). Adaptive Dynamic Programming Based Motion Control of Autonomous Underwater Vehicles. 2018 5th International Conference on Control, Decision and Information Technologies (pp. 966-971). Thessaloniki, Greece.

Yoo, B. \& Kim, J. (2016). Path optimization for marine vehicles in ocean currents using reinforcement learning. Journal of Marine Science and Technology. 21(2), 334-343.

Chen, C., Chen, X. Q., Ma, F., Zeng, X. J., \& Wang, J. (2019). A knowledge-free path planning approach for smart ships based on reinforcement learning. Ocean Engineering, 189, 106299.

Guo, S., Zhang, X., Zheng, Y., \& Du, Y. (2020). An Autonomous Path Planning Model for Unmanned Ships Based on Deep Reinforcement Learning. Sensors, 20(2), 426.

Larson, J., Bruch, M. \& Haltermana, R. (2007). Advances in autonomous obstacle avoidance for unmanned surface vehicles, AUVSI Unmanned Systems North America Conference (pp. 484-498), Washington, D.C., United States.

Simmons, R., Henriksen, L., Chrisman, L., \& Whelan, G. (1998). Obstacle avoidance and safeguarding for a lunar rover. Proceedings of the AIAA Forum on Advanced Developments in Space Robotics (pp. 1-9), Madison, United states,

Krishnamurthy, P., Khorrami, F., \& Ng, T. L. (2008). Obstacle avoidance for unmanned sea surface vehicles: a hierarchical approach. Proceedings of the 17th World Congress on the International Federation of Automatic Control (pp. 6798-6803), Seoul, Korea.

Tang, P., Zhang, R., Liu, D., Huang, L., Liu, G., \& Deng, T. (2015). Local reactive obstacle avoidance approach for high-speed unmanned surface vehicle. Ocean Engineering, 106, 128-140.

Fiorini, P., \& Shiller Z. (1998). Motion planning in dynamic environments using velocity obstacles, The International Journal of Robotics Research, 17(7), 760-772.

Kuwata, Y., Wolf, M. T., Zarzhitsky, D., \& Huntsberger, T. L. (2014). Safe Maritime Autonomous Navigation With COLREGS, Using Velocity Obstacles. IEEE Journal of Oceanic Engineering, 39 (1), 110-119.

Shiller, Z. (1999). Online sub-optimal obstacle avoidance, Proceedings 1999 IEEE International Conference on Robotics and Automation (pp. 335-340), Detroit, United States.

Gal, O., Shiller, Z., \& Rimon E. (2009). Efficient and Safe On-Line Motion Planning in Dynamic Environments. IEEE International Conference on Robotics and Automation (pp. 88-93), Kobe, Japan.

Zhuang, J., Luo, J., Liu, Y., Bucknall, R., Sun, H., \& Huang, C. (2019). Collision Avoidance for Unmanned Surface Vehicles based on COLREGS, 2019 5th International Conference on Transportation Information and Safety (ICTIS) (pp. 1418-1425), Liverpool, United Kingdom.

Song, A. L., Su, B. Y., Dong, C. Z., Shen, D. W., Xiang, E. Z., \& Mao, F. P. (2018). A two-level dynamic 
obstacle avoidance algorithm for unmanned surface vehicles. Ocean Engineering, 170, 351-360.

Bandyophadyay, T., Sarcione, L., \& Hover, F.S. (2010). A Simple Reactive Obstacle Avoidance Algorithm and Its Application in Singapore Harbor. Field and Service Robotics. Springer Tracts in Advanced Robotics, 62, 455-465.

Bertaska, I. R., Shah, B., Von Ellenrieder, K., Svec, P., Klinger, W., \& Sinisterra, A. J., et al. (2015). Experimental evaluation of automatically-generated behaviors for usv operations. Ocean Engineering, $106,496-514$. 